Ana Carolina da Cruz

\title{
Análise de normalização e significância em experimentos baseados em Proteômica
}

Dissertação apresentada ao Instituto de Matemática e Estatística da Universidade de São Paulo para obtenção do título de mestra em Estatística.

Orientadora: Prof ${ }^{\mathrm{a}}$. Dr ${ }^{\mathrm{a}}$. Julia Maria Pavan Soler Programa de pós-graduação em Estatística

São Paulo

2021 

Ana Carolina da Cruz

\title{
Análise de normalização e significância em experimentos baseados em Proteômica
}

\author{
Dissertação apresentada ao Instituto de \\ Matemática e Estatística da Universidade \\ de São Paulo para obtenção do título de \\ mestra em Estatística.
}

Banca Examinadora:

- Prof ${ }^{a}$. Dr ${ }^{\mathrm{a}}$. Julia Maria Pavan Soler (orientadora) - IME/USP

- Prof ${ }^{\mathrm{a}}$. Dr ${ }^{\mathrm{a}}$. Luzia Aparecida Trinca - UNESP

- Prof. Dr. George Freitas von Borries - UnB

Dissertação aprovada pela Banca Examinadora em 14 de julho de 2021.

São Paulo

2021 



\section{Agradecimentos}

Eu gostaria de agradecer todo o apoio que tive da minha família durante o processo de formação deste trabalho e da minha carreira acadêmica, tais pessoas foram fundamentais para que eu pudesse chegar até aqui. Além deles, gostaria de agradecer meus amigos em que compartilhei momentos difíceis e felizes durante o mestrado e graduação, mostrando o quanto é importante ter pessoas que te motivam e ajudam a conquistar seus sonhos.

Gostaria de agradecer a minha orientadora Prof ${ }^{\text {a }}$. Dr ${ }^{\mathrm{a}}$. Julia Maria Pavan Soler em que tive oportunidade de aprender e compartilhar conhecimento, uma pessoa super humorada e atenciosa, sempre motivando seus alunos a persistir com seus sonhos e mostrando tudo de incrível que podemos alcançar ao seguir na carreira acadêmica.

Além de tudo, gostaria de agradecer o meu orientador da graduação Prof. Dr. George von Borries em que tive a oportunidade de aprender tantas coisas e que fez com que eu decidisse seguir com o mestrado e agora doutorado. Foi em uma das disciplinas que fiz com ele, que tive certeza que me tornar uma Estatística era o que eu de fato queria alcançar.

Dito isso, só tenho o que agradecer por tudo que aprendi com os meus orientadores e professores da Universidade de Brasília e da Universidade de São Paulo. Além disso, gostaria de agradecer o o Conselho Nacional de Desenvolvimento Científico e Tecnológico - CNPq pela bolsa de estudos que tive durante o meu mestrado. 



\section{Resumo}

Da Cruz, A.C. Análise de normalização e significância em experimentos baseados em Proteômica. 2021. Dissertação de Mestrado - Instituto de Matemática e Estatística, Universidade de São Paulo, São Paulo.

A análise de dados ômicos vem se expandindo nos últimos anos e com isso tornouse necessário desenvolver técnicas estatísticas para análise desse tipo de dados. Pensando nos estudos proteômicos, baseados na análise de espectrometria de massas, nota-se que com a grande quantidade de informação coletada a utilização de técnicas de normalização e significância são fundamentais para comparação e identificação de variáveis com comportamento diferencial, podendo auxiliar no desenvolvimento de novos tratamentos. Este trabalho teve como objetivo propor novas técnicas de normalização e significância que levem em consideração a estrutura em que os dados foram coletados para comparar diferentes métodos de preparação de amostras (os quais serão então analisadas para quantificação das proteínas e peptídeos) em busca do mais preciso, além de interpretar as diferentes fontes de variação impostas pelo delineamento do experimento. Como resultados do estudo observou-se que as técnicas propostas neste trabalho foram eficazes na normalização dos dados e na identificação de variáveis significantes, observando ainda diferenças entre os resultados obtidos pelas técnicas propostas com os resultados baseados na metodologia de normalização TIC. Além disso, notou-se que o método IGD (em gel) no geral apresentou as menores quantificações e o método ISD (em solução) se mostrou o mais preciso.

Palavras-chave: proteômica; modelo misto heterocedástico; decomposição de matrizes; componentes principais; modelos gráficos probabilísticos. 



\section{Abstract}

Da Cruz, A.C. Normalization and significance analysis in Proteomics experiments. 2021. Masters Thesis - Institute of Mathematics and Statistics, University of São Paulo, São Paulo.

In the past years, omic data analysis has expanded, as a result, statistical techniques are being developed to study this kind of data. Concerning the amount of data generated by Mass Spectrometry based Proteomics experiments normalization and significance analysis are fundamental to compare and to find variables with differential abundance. This paper aims to propose new normalization and significance models that take the data's structure into account, to compare different preparation methods, which will be analyzed to quantify proteins and peptides, to find the most precise, and to explain the technical variation sources imposed by the experiment. It was found that the techniques proposed in this paper were efficient at their purposes, and was observed differences between results from these techniques with results from the normalization method TIC. Besides, IGD (in gel) had the lowest variable abundance, and ISD (in solution) was the preparation method most precise.

Keywords: proteomics; heterocedastic mixed model; matrix decomposition; principal components; probabilistic graph models. 



\section{Sumário}

1 Introdução 13

2 Histórico $\quad 15$

3 Descrição dos dados $\quad 19$

3.1 Objetivos . . . . . . . . . . . . . . . . . . . 21

3.2 Delineamento do Experimento . . . . . . . . . . . . . . . . 21

4 Metodologia $\quad 23$

4.1 Modelo de normalização . . . . . . . . . . . . . . . . . . . . . . . . 23

4.1 .1 Modelo Linear Clássico . . . . . . . . . . . . . . . . . . . . . 24

4.1.2 Modelo Linear Misto com erros homocedásticos . . . . . . . . 28

4.1.3 Modelo Linear Misto com erros heterocedásticos . . . . . . . . 38

4.2 Modelo de significância . . . . . . . . . . . . . . . . . . . . . . . . 42

4.3 Racional da Análise . . . . . . . . . . . . . . . . . . . . . . . . . . . 42

4.4 Análises Multivariadas . . . . . . . . . . . . . . . . . . . . . . 43

4.4.1 Análise de Componentes Principais . . . . . . . . . . . . . . 44

4.4.2 Análise de Grafos Probabilísticos . . . . . . . . . . . . . . . . 45

$\begin{array}{lll}5 & \text { Análise } & 47\end{array}$

$5.1 \quad$ Experimento SRM . . . . . . . . . . . . . . . . . 47

5.1 .1 Modelo de normalização . . . . . . . . . . . . . . . 51

5.1 .2 Modelo de significância . . . . . . . . . . . . . . . 55

5.2 Experimento DDA - noNorm Peptídeos . . . . . . . . . . 56

5.2 .1 Modelo de normalização . . . . . . . . . . . . . . 56

5.2 .2 Modelo de significância . . . . . . . . . . . . . . . . . . 57

5.3 Experimento DDA - TIC Peptídeos . . . . . . . . . . . . . . 61

5.3.1 Modelo de significância . . . . . . . . . . . . . . . . 62

5.4 Experimento DDA - noNorm Proteínas . . . . . . . . . . . . . 64

5.4 .1 Modelo de normalização . . . . . . . . . . . . . . . . . . . 64 
5.4 .2 Modelo de significância . . . . . . . . . . . . . . . 66

5.5 Experimento DDA - TIC Proteínas . . . . . . . . . . . . . 69

5.5.1 Modelo de significância . . . . . . . . . . . . 70

5.6 Análise dos experimentos . . . . . . . . . . . . . . . . . . . . . . . . . . . . . . . . . . . . . . . . .

5.7 Análises Multivariadas . . . . . . . . . . . . . . . . 73

5.7.1 Análise de Componentes Principais . . . . . . . . . . . 73

5.7.2 Análise de Grafos Probabilísticos . . . . . . . . . . . . 77

6 Considerações Finais $\quad 91$

$\begin{array}{ll}\text { Referências Bibliográficas } & 93\end{array}$

$\begin{array}{ll}\text { APÊNDICE A - Tabelas suplementares } & 97\end{array}$

A.1 Experimento SRM . . . . . . . . . . . . . . . . . . . . 97

A.2 Experimento DDA - noNorm Peptídeos . . . . . . . . . . . . . 102

A.3 Experimento DDA - TIC Peptídeos . . . . . . . . . . . . . 107

A.4 Experimento DDA noNorm Proteínas . . . . . . . . . . . . . 109

A.5 Experimento DDA - TIC Proteínas . . . . . . . . . . . . . . 114 


\section{Capítulo 1}

\section{Introdução}

A análise de dados ômicos vem se expandindo nos últimos anos, deixando de ser voltada somente à avaliação do genoma e partindo para outros níveis, como por exemplo proteoma, metaboloma e epigenoma, possibilitando assim avaliar de forma holística todo o sistema biológico.

Pensando nos estudos proteômicos tem-se que grande parte deles se baseiam na análise de espectrometria de massa, que consiste na identificação e quantificação de peptídeos e proteínas presentes em amostras biológicas por meio da ionização e fragmentação desses materiais. Contudo, existem diferentes métodos de espectrometria e de preparação de amostras que podem ser utilizados para quantificação das variáveis de interesse.

No estudo desenvolvido por Klont et al. [18] foram comparados diferentes métodos de preparação utilizados para quantificação e identificação de proteínas e peptídeos em estudos proteômicos. Os autores tinham como objetivo verificar a existência de um método de preparação universal a ser utilizado independente do tipo de amostra avaliada. Neste estudo foram encontradas diferenças nas quantificações das proteínas e peptídeos no que diz respeito à perda, variabilidade e características biológicas das proteínas e peptídeos identificados.

Dessa forma, este trabalho visa dar continuidade ao estudo desenvolvido por eles [18], propondo técnicas estatísticas que permitam comparar os diferentes métodos utilizados quanto à precisão e quantificação dos peptídeos e proteínas avaliadas, porém considerando a estrutura do delineamento do experimento, possibilitando assim identificar e interpretar as diferentes fontes de variação impostas pela estruturação dos dados.

Primeiramente serão propostas técnicas de normalização para estudos proteômicos baseados em modelos de regressão, com intuito de comparar os diferentes métodos de preparação de amostras avaliados no estudo e identificar as diferentes fontes de 
variação técnica impostas pelo delineamento. Em seguida, será proposto uma forma de se retirar tais fontes de variação dos dados para avaliar a significância das variáveis quantificadas em diferentes condições de interesse.

Além disso, as metodologias de normalização propostas neste trabalho serão comparadas com uma técnica comumente utilizada na Bioinformática para normalização da abundância de proteínas e peptídeos em amostras biológicas, com intuito de se avaliar o quanto a técnica de normalização escolhida pode influenciar nos resultados.

Por fim, com base nos resultados obtidos pelas técnicas propostas será avaliada ainda a estrutura dos dados em cada experimento por meio de análises multivariadas para identificar possíveis agrupamentos nos dados e grafos de causalidade entre os peptídeos e as proteínas avaliadas. 


\section{Capítulo 2}

\section{Histórico}

A análise de dados ômicos avalia a estrutura e o funcionamento de moléculas biológicas, considerando diferentes níveis do sistema molecular: genético (Genômica), proteico (Proteômica), metabólico (Metabolômica), dentre outros. O termo ômico se refere ao fato de que todas ou quase todas as instâncias do espaço molecular avaliado estão sendo medidas. Dessa forma, tem-se uma visão holística do sistema biológico.

Durante muitos anos, experimentos ômicos focavam principalmente na análise do genoma. Contudo, nas últimas décadas esse tipo de análise vem se expandindo para avaliar outros níveis, tais como transcriptoma, proteoma, lipoma, epigenoma e metaboloma. Sendo que, atualmente, com o avanço tecnológico, além da criação de novos estudos, tornou-se possível ainda avaliar diferentes níveis de uma amostra biológica simultaneamente (estudos multiômicos).

Pensando no estudo voltado à constituição proteica do organismo, tem-se como objetivo avaliar o proteoma, entendendo as funções que ocorrem no nível proteico, tais como: as modificações pós-traducionais, interações proteína-proteína e outras, uma vez que, por meio da análise da expressão gênica, tais informações acabam sendo perdidas na análise do RNA. Dessa forma, tem-se por meio da Proteômica a possibilidade de caracterizar a localização, abundância, modificações pós-traducionais e interações biomoleculares das proteínas presentes em um organismo. Um dos objetivos dessa análise é encontrar biomarcadores que ajudem no diagnóstico precoce e no tratamento de doenças [1].

Em relação à metodologia utilizada neste tipo de análise (Figura 2.1), os passos comuns a serem seguidos consistem em inicialmente avaliar uma amostra biológica, por exemplo, um tecido hepático. Sendo que, dessa amostra são extraídas as proteínas, caso a análise seja realizada no nível de proteína, as quais são separadas por eletroforese unidimensional (1 - DE) ou bidimensional (2 - DE) ou por cromatografia líquida (LC). No entanto, se estivermos avaliando os peptídeos da amostra, as proteí- 
nas extraídas passam por um processo de digestão, dado pela utilização de proteases (enzimas) que quebram as ligações peptídicas entre os aminoácidos das proteínas, com o intuito de se obter uma mistura de peptídeos e seguir com a análise. Após a separação, o conteúdo passa por um processo de ionização e de fragmentação. Por meio dos fragmentos obtidos é realizada a análise de massas e detecção de peptídeos por meio da espectrometria de massa (MS). Na fase final, para identificação dos peptídeos e ou proteínas presentes na amostra, são utilizadas bases de dados disponíveis para consulta e anotação.

Figura 2.1 - Um Fluxo típico em estudos proteômicos

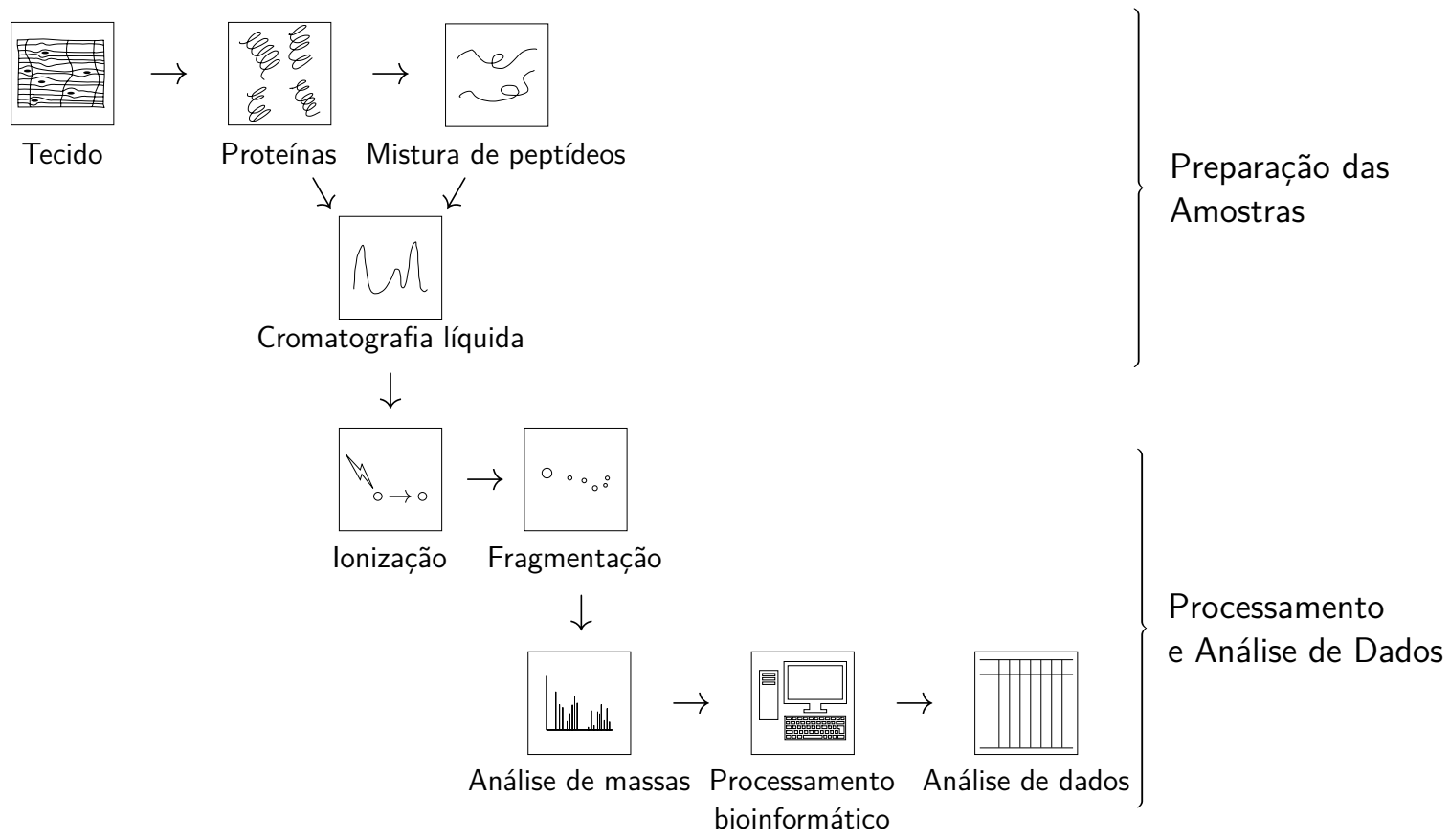

Atualmente, tem-se duas metodologias comumente utilizadas em estudos proteômicos: bottom-up (também denominada de shotgun) e a top-down. A primeira consiste em avaliar os peptídeos presentes na amostra, seguindo o fluxo apresentado na Figura 2.1. A segunda metodologia consiste em avaliar as proteínas e não os peptídeos, as quais também passam por uma análise de espectrometria de massa. A metodologia shotgun possui diversas vantagens, como reprodutibilidade e sensibilidade, porém, por se avaliar os peptídeos e não as proteínas, as modificações pós-traducionais acabam por não ser avaliadas.

A identificação e quantificação das proteínas e/ou peptídeos é dada por meio da espectrometria de massa (MS), um procedimento bastante utilizado em estudos proteômicos por apresentar sensitividade e ser versátil na identificação e quantificação de proteínas e/ou peptídeos. A MS consiste em identificar a estrutura molecular de 
um composto por meio da ionização e fragmentação em pequenos íons, em seguida, analisando o resultado obtido pela ionização e fragmentação, é deduzido a ordem em que os fragmentos estão unidos, de tal forma que é possível identificar peptídeos e/ou proteínas por meio da comparação com as informações presentes em bases de dados.

Tratando-se especificamente dos procedimentos realizados durante a análise MS, tem-se que após a digestão da proteína ou de um conjunto de proteínas em uma mistura de peptídeos, tal conteúdo é analisado por meio de um espectrômetro de massa para quantificar a razão massa/carga desse composto, sendo que por meio dessa quantificação é possível identificar os peptídeos presentes na amostra. Todavia, muitas vezes é de interesse identificar as proteínas presentes na amostra biológica e não somente os peptídeos, sendo assim, tem-se a necessidade de inferir por meio da identificação dos peptídeos, as proteínas presentes na amostra. Para tornar esse processo de identificação mais preciso é comum analisar os peptídeos que foram identificados como mais abundantes em uma primeira análise MS, e identificar as cadeias de peptídeos por meio de uma segunda MS ( $\mathrm{MS}^{2}$ - tandem MS), dessa forma é possível obter a sequência completa dos aminoácidos dos peptídeos e/ou proteína e até mesmo as modificações pós-traducionais.

Além disso, é interessante ressaltar que, durante o processo de identificação das proteínas e/ou peptídeos, pode ser de interesse a identificação de um certo conjunto específico de proteínas e/ou peptídeos, para identificar, por exemplo, biomarcadores. Esse tipo de análise pode ser realizada por meio do Monitoramento de Reação Selecionada (SRM, do inglês Targeted Mass Spectrometry), que consiste em identificar e quantificar componentes com propriedades de fragmentação conhecidas. Dessa forma, a SRM seleciona os peptídeos por meio de suas medidas de razão/carga dadas pela primeira análise de massa, depois esses peptídeos selecionados são fragmentados em pequenos componentes e avaliados por um segundo espectrômetro de massa para identificar os componentes de interesse.

Outra técnica de quantificação proteômica bastante utilizada é a Aquisição de Dados Dependentes (DDA, do inglês Data Dependent Acquisition) e consiste em identificar de forma simultânea milhares de proteínas e/ou peptídeos em um material biológico. Assim como para SRM, são selecionados peptídeos na primeira análise de massa, porém ao invés de selecionar peptídeos específicos por meio de suas medidas de razão massa/carga, na DDA os peptídeos selecionados são os mais abundantes e, por meio da segunda análise de massa, é possível identificar tais peptídeos e proteínas presentes na amostra biológica.

Atualmente com a expansão desse tipo de análise, tem-se como resultado uma grande quantidade de informações complexas que buscam responder diversas ques- 
tões relacionadas a diferentes problemas. Dessa forma, a busca por metodologias estatísticas que possam avaliar tais informações, levando em consideração a complexidade dos dados ainda continua em aberto [8]. 


\section{Capítulo 3}

\section{Descrição dos dados}

No estudo desenvolvido por Klont [18], foram comparados diferentes métodos de preparação utilizados para identificação e quantificação de proteínas e peptídeos em estudos proteômicos (bottom-up) baseados em espectrometria de massa em busca de identificar a existência de um possível método que poderia ser considerado em todos os cenários. Para comparação foram avaliadas a perda relativa de peptídeos e proteínas identificados nas amostras em cada método, a precisão das quantificações e o potencial de descoberta. Nessa comparação, foi possível observar diferenças entre os métodos quanto à precisão, quantificação e características biológicas das proteínas e peptídeos quantificados pelos experimentos realizados. Além disso, foi ressaltada a importância em selecionar o método mais adequado considerando a estrutura dos dados e métodos a serem estudados, ao invés de utilizar sempre o mesmo método de preparação - normalmente o ISD (em solução) —, sem se atentar ao tipo de amostra biológica sendo avaliada.

Dito isso, este trabalho busca dar continuidade ao estudo realizado em Klont [18], buscando propor metodologias estatísticas que considerem a estrutura dos dados para comparar os diferentes métodos de preparação estudados, em busca de identificar o que produz a maior quantificação de proteínas ou peptídeos e o de maior precisão. Além disso, será proposto para análise de significância um modelo estatístico baseado nas observações sem variação sistemática para identificação de variáveis com comportamento diferencial entre os métodos avaliados.

Neste estudo serão comparados, assim como no estudo do Klont [18], quatro métodos de preparação (digestão) comumente utilizados em estudos proteômicos para identificação de proteínas e peptídeos em amostras biológicas, quais sejam: em solução (ISD), no filtro (OFD), em gel (IGD) e na paleta (OPD). Serão avaliados dois métodos de espectrometria de massa: SRM e DDA (MS - Target SRM e MS - Shotgun DDA). As amostras biológicas foram coletadas a partir de três tecidos humanos cancerígenos 
das regiões da cabeça e do pescoço, mais precisamente dos pólipos nasais (NP), da glândula parótida $(\mathrm{P})$ e das amídalas $(\mathrm{T})$.

Além dos três tecidos coletados, foi obtida uma mistura dos tecidos, denominada pool (Pool). A utilização desse tipo de amostra, representada por uma mistura das demais, normalmente está relacionada ao custo do experimento. Aliás, a utilização da agregação de observações como uma nova observação é comum em estudos moleculares e visa aumentar a habilidade de encontrar diferenças entre grupos por meio da redução da variação existente entre as mesmas observações. [17]

O experimento foi construído hierarquicamente da seguinte maneira: para cada um dos tecidos foram obtidas quatro subamostras (réplicas), as quais foram alocadas aleatoriamente a cada um dos métodos de preparação. Após os procedimentos de preparação, o produto final de cada método é uma mistura de peptídeos e para para cada uma das 16 misturas foram obtidas 10 réplicas, as quais foram alocadas aleatoriamente de forma balanceada a cada método de espectrometria de massa (SRM, DDA) para a quantificação das proteínas e peptídeos.

Na tabela 3.1, temos a estruturação dos dados conforme o delineamento do experimento. Considerando cada método de espectrometria separadamente, temos 80 unidades experimentais.

Tabela 3.1 - Estrutura dos dados

\begin{tabular}{|c|c|c|c|c|c|c|c|c|c|c|c|}
\hline \multirow{2}{*}{ Tecido } & \multirow{2}{*}{ Método } & \multicolumn{5}{|c|}{ Experimento SRM } & \multicolumn{5}{|c|}{ Experimento DDA } \\
\hline & & R1 & $\mathrm{R} 2$ & R3 & $\mathrm{R} 4$ & $\mathrm{R} 5$ & R6 & $\mathrm{R} 7$ & $\bar{R} 8$ & R9 & $\mathrm{R} 10$ \\
\hline \multirow{4}{*}{ NP } & ISD & & & & & & & & & & \\
\hline & OPD & & & & & & & & & & \\
\hline & IGD & & & & & & & & & & \\
\hline & OFD & & & & & & & & & & \\
\hline \multirow{4}{*}{$\mathrm{P}$} & ISD & & & & & & & & & & \\
\hline & OPD & & & & & & & & & & \\
\hline & IGD & & & & & & & & & & \\
\hline & OFD & & & & & & & & & & \\
\hline \multirow{4}{*}{$\mathrm{T}$} & ISD & & & & & & & & & & \\
\hline & OPD & & & & & & & & & & \\
\hline & IGD & & & & & & & & & & \\
\hline & OFD & & & & & & & & & & \\
\hline \multirow{4}{*}{ Pool } & ISD & & & & & & & & & & \\
\hline & $\overline{\mathrm{OPD}}$ & & & & & & & & & & \\
\hline & IGD & & & & & & & & & & \\
\hline & OFD & & & & & & & & & & \\
\hline
\end{tabular}

Para o método MS - SRM, os peptídeos (variáveis) a serem quantificados foram previamente especificados. Dessa forma, temos como resultado do experimento uma 
base de dados $\mathbf{Y}_{80 \times p}$, em que $p$ representa cada variável quantificada e o número de linhas é dado pelo número de realizações do experimento $(4 \times 4 \times 5=80$, quatro tecidos, quatro métodos e cinco réplicas).

Para o método MS - DDA, as variáveis (peptídeos e proteínas) obtidas foram aleatórias, ou seja, não foram especificadas previamente, resultando em duas bases de dados $\mathbf{Y}_{80 \times q}$ e $\mathbf{Y}_{80 \times s}$, em que $q$ e $s$ representam os peptídeos e as proteínas identificadas e quantificadas no experimento, respectivamente.

Neste trabalho serão avaliadas cada base de dados obtida pelos métodos de espectrometria de massa (SRM - peptídeos, DDA - peptídeos e DDA - proteínas) separadamente. Dessa forma, para cada recorte serão ajustados modelos estatísticos para realização do pré-processamento dos dados, com o intuito de se retirar variabilidade indesejável imposta pela forma de coleta dos dados. Além disso, serão estimados modelos de significância para identificação dos peptídeos e proteínas com expressão diferencial entre os tecidos e métodos de preparação.

\subsection{Objetivos}

Considerando as quantificações obtidas por cada experimento avaliado, este trabalho tem como objetivos:

- Avaliar a existência de possível diferença entre os métodos de preparação utilizados nos experimentos;

- Identificar qual método possui a maior quantificação de proteínas e ou peptídeos e qual o mais preciso;

- Estimar e interpretar as fontes de variação técnica impostas pelo delineamento dos experimentos.

Além disso, será de interesse ainda comparar os resultados obtidos pelo método de normalização proposto nesse trabalho com o método TIC (mais detalhes serão discutidos no Capítulo 4), bastante utilizado na Bioinformática, avaliando assim, o impacto que o modelo de normalização escolhido tem na análise dos peptídeos e proteínas avaliados em estudos proteômicos.

\subsection{Delineamento do Experimento}

Para propor modelos estatísticos que comparem os diferentes métodos de preparação de amostras e que considerem a estrutura dos dados experimentos é importante 
entender a estrutura do delineamento do experimento: as unidades amostrais e experimentais, fatores experimentais, o processo de randomização e a estrutura da variável resposta de interesse [9].

Sendo assim, considerando a descrição dos dados realizada anteriormente, temos que a estrutura das unidades experimentais (que foram aleatorizadas aos tratamentos) caracteriza um modelo de medidas repetidas, pois a mesma amostra de tecido foi avaliada nos diferentes métodos de preparação e espectrometria de massa.

Nota-se que as unidades experimentais foram aleatorizadas aos tratamentos sob uma estrutura hierárquica, em que o nível 1 está representado pelos tecidos (NP,P,T e Pool), o nível 2 é dada pelas subamostras atribuídas aos métodos dentro de tecido e o nível 3 corresponde às réplicas por subamostra dentro de método e dentro de cada tecido.

O delineamento do experimento pode assim ser identificado como um Split-SplitPlot, com quatro plots completos, representados pelos quatro tecidos (NP, P, T, Pool), cada um contendo quatro subplots, os quais contêm 10 réplicas cada.

Deste modo, estão sendo avaliados três fatores principais no experimento: tecido, método de preparação e método de espectrometria de massa completamente cruzados em um esquema fatorial $4 \times 4 \times 2$. Observa-se que o fator tecido não possui réplica genuína e que cada combinação de tratamento foi aplicada às subunidades experimentais.

A análise será realizada separadamente para cada método de espectrometria de massa, sendo que, para cada recorte, temos que o fator método dentro de tecido possui cinco réplicas e é definido como efeito fixo de interesse no estudo. Além disso, como mencionado anteriormente, para o método MS - Target SRM são quantificados um número fixo de variáveis, no caso, peptídeos (alvo) de interesse. Já para o método MS - Shotgun DDA, em que é realizada uma varredura completa, as variáveis quantificadas são aleatórias e é possível medir diferentes níveis de profundidade (peptídeos e proteínas). Espera-se que os resultados obtidos pelo método DDA sejam extremamente desbalanceados (na estrutura das variáveis quantificadas nas réplicas) quando comparado ao SRM. Todavia, para reduzir essa diferença, serão consideradas somente as variáveis que tiverem sido quantificadas em pelo menos três das cinco réplicas dentro de cada método aninhado a tecido.

No Capítulo 4 serão descritos em detalhes o racional da análise e as metodologias propostas neste trabalho. Já no Capítulo 5 serão apresentados os resultados obtidos pela análise dos dados, buscando responder às questões de interesse deste estudo. 


\section{Capítulo 4}

\section{Metodologia}

Um dos principais focos em estudos proteômicos consiste em identificar proteínas com expressão diferencial quanto à suas abundâncias. Os instrumentos utilizados nesses estudos são bastante sensíveis para possibilitar a detecção de mudanças expressivas nas amostras, porém essa sensibilidade também capta mudanças devido à experimentação, preparação da amostra e instrumentação. Dessa forma, é crucial ter em mãos dados fidedignos, sem viés ou erros sistemáticos. Portanto, a utilização de um processo de normalização é fundamental, pois remove tais variações indesejáveis e mantém a acurácia e precisão das conclusões obtidas pelo estudo.

Muitas vezes, nesses estudos é comum mensurar centenas ou milhares de peptídeos e proteínas em cada amostra. Devido a isso, na análise de significância, as comparações múltiplas que permitem identificar mudanças expressivas nas proteínas, devem ser realizadas de forma correta para assegurar que os resultados obtidos representem de fato mudanças reais no proteoma e não falsos positivos.

Neste Capítulo serão apresentados o racional da análise, os modelos propostos para normalização e significância dos resultados dos experimentos. Além disso, serão apresentados, para cada modelo, códigos em $\mathrm{R}$ que possibilitem realizar os ajustes e estimação envolvidos na análise proposta [29] [3].

\subsection{Modelo de normalização}

Usualmente em estudos baseados em experimentos de espectrometria de massa as amostras avaliadas são processadas em diferentes passos, podendo ser no mesmo instrumento ou em instrumentos diferentes. Sendo assim, torna-se necessário ajustar os íons quantificados em diferentes realizações para uma escala comparável. Esse procedimento é chamado de normalização (dos dados proteômicos). O objetivo da normalização é retirar a variabilidade indesejável devido à técnica, mantendo varia- 
ções devido às fontes biológicas.

Um dos métodos mais utilizados para essa finalidade nesse tipo de estudo consiste em ajustar cada amostra por meio da sua corrente de íon total (TIC, do inglês Total Ion Current), ou seja, cada variável quantificada na amostra, tem sua abundância divida pela intensidade total da amostra. Esse método é bastante popular, pois não é computacionalmente intensivo e simples de calcular.

No entanto, esse tipo de método é influenciado por variáveis que possuem observações com alta ionização. Além disso, o método TIC considera que a maioria das variáveis não sofre influência sobre as condições que estão sendo testadas e, muitas vezes, além de retirarem a variação sistemática, acabam retirando também a variação biológica de interesse.

Sendo assim, tem-se a necessidade de utilizar outras técnicas que sejam mais assertivas para normalização dos dados em estudos proteômicos. Os modelos de regressão podem ser utilizados como procedimentos de normalização [38]. A grande vantagem é que permitem controlar (filtrar) fontes de variação conhecidas provenientes do delineamento do experimento para o ajuste dos dados, sendo possível assim retirar vieses e variações sistemáticas por meio da utilização dos resíduos do modelo ajustado. Portanto, neste trabalho, serão propostos três métodos de normalização baseados em modelos de regressão linear para obtenção de observações sem variação técnica.

Neste contexto, os possíveis modelos que podem ser propostos para realização da normalização são: (1) Modelo Linear Clássico, (2) Modelo Linear Misto com erros homocedásticos, e (3) Modelo Linear Misto com erros heterocedásticos. Esses modelos atendem diversas situações por serem flexíveis quanto à estrutura dos dados a serem avaliados. O leitor já familiarizado com essas metodologias pode se encaminhar para a seção 4.3 com o Racional da Análise.

\subsubsection{Modelo Linear Clássico}

O Modelo Linear Clássico é um dos modelos mais simples para normalização dos dados por considerar somente efeitos fixos e muitas vezes homocedasticidade dos erros. Tal modelo pode ser definido da seguinte forma,

$$
y_{i j k l}=\mu+\beta_{j}+\epsilon_{i j k l}
$$

em que,

$$
\epsilon_{i j k l} \sim N_{1}\left(0 ; \sigma^{2}\right)
$$

$y_{i j k l}$ representa a quantificação da l-ésima variável avaliada na $k$-ésima réplica, no 
$j$-ésimo método de preparação e no $i$-ésimo tecido.

Além disso, temos que $\mu$ corresponde a média geral, $\beta_{j}$ o efeito do $j$-ésimo método de preparação, e os erros $\epsilon_{i j k l}$ independentes e identicamente distribuídos.

Considerando que $E\left(\epsilon_{i j k l}\right)=0$ e $\operatorname{Var}\left(\epsilon_{i j k l}\right)=\sigma^{2}$, temos que

$$
\begin{gathered}
\mathrm{E}\left(y_{i j k l}\right)=\mu+\beta_{j}, \\
\operatorname{Var}\left(y_{i j k l}\right)=\sigma^{2} .
\end{gathered}
$$

O Modelo (4.1) pode ser reescrito em forma matricial, facilitando a obtenção dos estimadores dos parâmetros do modelo. Sendo assim, temos abaixo o modelo em sua forma matricial,

$$
\mathbf{y}=\mathbf{X} \boldsymbol{\beta}+\boldsymbol{\epsilon},
$$

sendo que, $\mathbf{y}$ representa o vetor de respostas, $\mathbf{X}$ é a matriz de delineamento dos efeitos fixos $\boldsymbol{\beta}, \boldsymbol{\beta}$ representa o vetor dos efeitos fixos do modelo e $\boldsymbol{\epsilon}$ é o vetor de erros.

A estimação dos parâmetros $\boldsymbol{\beta}$ pode ser obtida pelo Método dos Mínimos Quadrados bem como pelo de Máxima Verossimilhança.

\section{Método dos Mínimos Quadrados}

Considerando o modelo na forma matricial (4.5), temos que a estimação por meio do Método dos Mínimos Quadrados consiste em minimizar a soma de quadrados $Q$ da distância entre $\mathbf{y}$ e seu valor esperado $\mathrm{E}(\mathbf{y})=\mathbf{X} \boldsymbol{\beta}$.

Dessa forma, os estimadores de $\boldsymbol{\beta}$ representam os valores que minimizam $Q=$ $(\mathbf{y}-\mathbf{X} \boldsymbol{\beta})^{T}(\mathbf{y}-\mathbf{X} \boldsymbol{\beta})$.

Sendo assim, temos que

$$
\begin{aligned}
Q & =\left(\mathbf{y}^{\mathbf{T}}-\boldsymbol{\beta}^{\mathbf{T}} \mathbf{X}^{T}\right)(\mathbf{y}-\mathbf{X} \boldsymbol{\beta}) \\
& =\mathbf{y}^{\mathbf{T}} \mathbf{y}-\mathbf{y}^{\mathbf{T}} \mathbf{X} \boldsymbol{\beta}-\boldsymbol{\beta}^{\mathbf{T}} \mathbf{X}^{T} \mathbf{y}+\boldsymbol{\beta}^{\mathbf{T}} \mathbf{X}^{T} \mathbf{X} \boldsymbol{\beta} \\
& =\mathbf{y}^{\mathbf{T}} \mathbf{y}-2 \mathbf{y}^{\mathbf{T}} \mathbf{X} \boldsymbol{\beta}+\boldsymbol{\beta}^{\mathbf{T}} \mathbf{X}^{T} \mathbf{X} \boldsymbol{\beta} .
\end{aligned}
$$

Portanto, o valor que minimiza $Q$ é dado pela solução da equação

$$
\frac{\partial Q}{\partial \boldsymbol{\beta}}=0 \Longrightarrow-2 \mathbf{X}^{T} \mathbf{y}+2 \mathbf{X}^{T} \mathbf{X} \boldsymbol{\beta}=0
$$

uma vez que,

$$
\frac{\partial(\mathbf{A} \boldsymbol{\theta})}{\partial \boldsymbol{\theta}}=\mathbf{A}^{T}
$$


e

$$
\frac{\partial\left(\boldsymbol{\theta}^{\mathbf{T}} \mathbf{A} \boldsymbol{\theta}\right)}{\partial \boldsymbol{\theta}}=\left(\mathbf{A}+\mathbf{A}^{T}\right) \boldsymbol{\theta}=2 \mathbf{A} \boldsymbol{\theta}
$$

se $\mathbf{A}$ for simétrica.

Logo,

$$
\hat{\boldsymbol{\beta}}=\left(\mathbf{X}^{T} \mathbf{X}\right)^{-1} \mathbf{X}^{T} \mathbf{y}
$$

se $\mathbf{X}$ for de posto completo.

\section{Máxima Verossimilhança}

Levando em consideração o modelo descrito em (4.5), temos que

$$
\mathbf{y} \sim N_{N}\left(\mathbf{X} \boldsymbol{\beta} ; \sigma^{2} \mathbf{I}\right)
$$

A função de verossimilhança para o modelo é a densidade de probabilidade para os dados, dado os parâmetros, porém é vista, como uma função dos parâmetros com os dados fixos. Portanto, temos

$$
L\left(\boldsymbol{\beta}, \sigma^{2} \mid \mathbf{y}\right)=(2 \pi)^{-\frac{N}{2}}\left(\sigma^{2}\right)^{-\frac{N}{2}} \exp \left[-\frac{1}{2 \sigma^{2}}(\mathbf{y}-\mathbf{X} \boldsymbol{\beta})^{T}(\mathbf{y}-\mathbf{X} \boldsymbol{\beta})\right] .
$$

O logaritmo da função de verossimilhança fica expresso como

$$
l\left(\boldsymbol{\beta}, \sigma^{2} \mid \mathbf{y}\right)=-\frac{N}{2} \log (2 \pi)-\frac{N}{2} \log \sigma^{2}-\frac{1}{2 \sigma^{2}}\left[(\mathbf{y}-\mathbf{X} \boldsymbol{\beta})^{T}(\mathbf{y}-\mathbf{X} \boldsymbol{\beta})\right]
$$

Considerando a função log-verossimilhança do modelo (4.5), temos que a estimação dos parâmetros do modelo é dada pela resolução das seguintes equações

$$
\begin{gathered}
\frac{\partial l}{\partial \boldsymbol{\beta}}=0 \Longrightarrow \mathbf{X}^{T}(\mathbf{y}-\mathbf{X} \boldsymbol{\beta})=0 \\
\frac{\partial l}{\partial \sigma^{2}}=0 \Longrightarrow-\frac{N}{2 \sigma^{2}}+\frac{1}{2 \sigma^{4}}(\mathbf{y}-\mathbf{X} \boldsymbol{\beta})^{T}(\mathbf{y}-\mathbf{X} \boldsymbol{\beta})=0 .
\end{gathered}
$$

Logo, temos que os estimadores de $\sigma^{2}$ e $\boldsymbol{\beta}$ são dados por

$$
\hat{\boldsymbol{\beta}}=\left(\mathbf{X}^{T} \mathbf{X}\right)^{-1} \mathbf{X}^{T} \mathbf{y}
$$

$\mathrm{e}$

$$
\hat{\sigma}^{2}=\frac{(\mathbf{y}-\mathbf{X} \hat{\boldsymbol{\beta}})^{T}(\mathbf{y}-\mathbf{X} \hat{\boldsymbol{\beta}})}{N} .
$$




\section{Resíduos}

Tendo em vista que os modelos utilizados para normalização visam retirar efeitos indesejáveis, temos que os resíduos desempenham um papel importante nesses modelos, pois representam os valores da variável de interesse sem os efeitos impostos pela técnica utilizada.

Portando para o modelo (4.1), os resíduos podem ser obtidos da seguinte maneira,

$$
\hat{\epsilon}_{i j k l}=y_{i j k l}-\hat{y}_{i j k l}
$$

em que $\hat{y}_{i j k l}$ corresponde ao valor ajustado de $y_{i j k l}$ pelo modelo.

Em termos matriciais, temos que

$$
\begin{aligned}
\hat{\mathbf{y}} & =\mathbf{X} \hat{\boldsymbol{\beta}} \\
& =\mathbf{X}\left(\mathbf{X}^{T} \mathbf{X}\right)^{-1} \mathbf{X}^{T} \mathbf{y} \\
& =\mathbf{H y},
\end{aligned}
$$

com $\mathbf{H}=\mathbf{X}\left(\mathbf{X}^{T} \mathbf{X}\right)^{-1} \mathbf{X}^{T}$ uma matriz simétrica e idempotente. Logo, os resíduos são dados por

$$
\begin{aligned}
\hat{\epsilon} & =\mathbf{y}-\hat{\mathbf{y}} \\
& =\mathbf{y}-\mathbf{H} \mathbf{y} \\
& =\left(\mathbf{I}_{N}-\mathbf{H}\right) \mathbf{y} .
\end{aligned}
$$

\section{Ferramenta R - função lm}

Para realização da análise de normalização via o ajuste desse modelo, que consiste em obter, por exemplo, os valores estimados dos parâmetros e os resíduos, será utilizado o software estatístico R. Sendo possível realizar a análise por meio da função lm do pacote stats.

Abaixo temos alguns comandos para obtenção das estimativas dos parâmetros e dos resíduos do modelo.

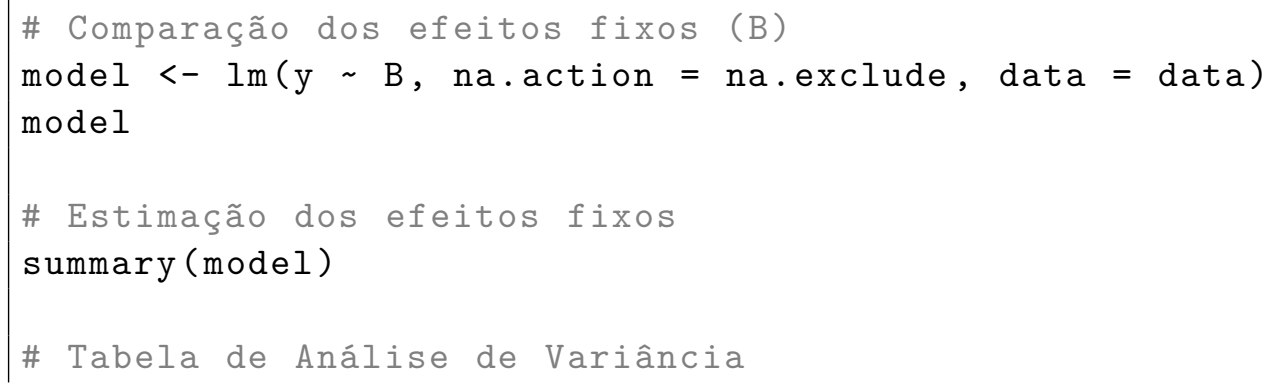




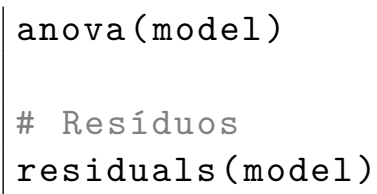

\subsubsection{Modelo Linear Misto com erros homocedásticos}

O Modelo Linear Misto pode ser adotado para levar em consideração a estrutura SplitSplit-Plot do experimento, sendo que, devido à estruturação hierárquica das unidades experimentais e do processo de randomização, pode-se definir efeitos aleatórios para cada nível hierárquico presente no delineamento. Portanto, pensando na estrutura do experimento descrito no Capítulo 3 , temos que para o nível 1, é definida a variável $\mathbf{u}_{1}$ correspondente ao efeito aleatório entre os tecidos. Para o nível 2, define-se a variável $\mathbf{u}_{2}$, correspondente ao efeito aleatório entre as subamostras aninhadas aos seus correspondentes tecidos.

Portanto, pode-se definir o modelo da seguinte maneira:

$$
y_{i j k l}=\mu_{i j}+\beta_{j}+\epsilon_{i j k l}
$$

em que,

$$
\begin{gathered}
\epsilon_{i j k l} \sim N_{1}\left(0 ; \sigma^{2}\right), \\
\mu_{i j}=\mu+u_{1 i}+u_{2 j(i)},
\end{gathered}
$$

$y_{i j k l}$ representa a quantificação da $l$-ésima variável avaliada na $k$-ésima réplica, no $j$-ésimo método de preparação e no $i$-ésimo tecido.

Além disso, temos que: $\mu$ corresponde à média geral, $\beta_{j}$ o efeito do $j$-ésimo método de preparação, $u_{1 i}$ o efeito aleatório do $i$-ésimo tecido, $u_{2 j(i)}$ o efeito aleatório entre a mistura de peptídeos obtida pelo $j$-ésimo método de preparação aninhado ao $i$-ésimo tecido e os erros $\epsilon_{i j k l}$ são independentes e identicamente distribuídos.

Levando em consideração que as observações que compartilham o mesmo efeito aleatório são correlacionadas. Pode-se definir as distribuições condicionais e marginais das respostas. Sendo que os valores ajustados e os resíduos podem ser obtidos com base nas distribuições condicionais ou marginais.

Diferentemente dos Modelos Lineares de efeitos fixos, os Modelos Lineares Mistos por meio da incorporação de efeitos aleatórios consideram a correlação existente entre as observações dentro de um mesmo grupo.

Nesta subsecção são apresentadas a formulação dos Modelos Lineares Mistos com um e dois níveis e a estimação dos parâmetros por Máxima Verossimilhança e Máxima 
Verossimilhança Restrita.

\section{Modelo Linear Misto com um único nível}

Considerando o modelo proposto por Laird [19], temos que o vetor de repostas $n_{i^{-}}$ dimensional $\mathbf{y}_{i}$ para o $i$-ésimo grupo pode ser descrito como

$$
\mathbf{y}_{i}=\mathbf{X}_{i} \boldsymbol{\beta}+\mathbf{Z}_{i} \mathbf{u}_{i}+\boldsymbol{\epsilon}_{i}, \quad i=1, \ldots, M
$$

Temos que,

$$
\begin{aligned}
\boldsymbol{\epsilon}_{i} & \sim N\left(\mathbf{0} ; \sigma^{2} \mathbf{I}\right), \\
\mathbf{u}_{i} & \sim N(\mathbf{0} ; \boldsymbol{\Psi}),
\end{aligned}
$$

$\Psi$ é positiva-definida.

Além disso, $\boldsymbol{\beta}$ é o vetor de efeitos fixos $p$-dimensional, $\mathbf{u}_{i}$ representa o vetor de efeitos aleatórios $q$-dimensional, $\mathbf{X}_{i_{n_{i} \times p}}$ e $\mathbf{Z}_{i_{n_{i} \times q}}$ são conhecidas e representam respectivamente as matrizes de delineamento dos efeitos fixos e aleatórios e $\boldsymbol{\epsilon}_{i}$ é o vetor $n_{i}$-dimensional de erro intragrupo.

Os erros $\boldsymbol{\epsilon}_{i}$ e os efeitos aleatórios $\mathbf{u}_{i}$ são independentes para diferentes grupos e entre si no mesmo grupo.

O modelo em (4.30) pode ser reescrito como

$$
\begin{aligned}
\mathbf{y}_{i} & =\mathbf{X}_{i} \boldsymbol{\beta}+\mathbf{Z}_{i} \mathbf{u}_{i}+\boldsymbol{\epsilon}_{i} \\
& =\mathbf{X}_{i} \boldsymbol{\beta}+\boldsymbol{\epsilon}_{i}^{*}, \quad i=1, \ldots, M
\end{aligned}
$$

em que,

$$
\boldsymbol{\epsilon}_{i}^{*}=\mathbf{Z}_{i} \mathbf{u}_{i}+\boldsymbol{\epsilon}_{i}
$$

Devido ao fato de $\boldsymbol{\epsilon}_{i}^{*}$ representar uma soma de duas Normais Multivariadas independentes, tem-se que

$$
\boldsymbol{\epsilon}_{i}^{*} \sim N_{M}\left(\mathbf{0} ; \sigma^{2} \boldsymbol{\Sigma}_{i}\right)
$$

em que

$$
\boldsymbol{\Sigma}_{i}=\mathbf{I}+\frac{\mathbf{Z}_{i} \Psi \mathbf{Z}_{i}^{T}}{\sigma^{2}}
$$

Sendo assim, a distribuição marginal de $\mathbf{y}_{i}$ é Normal Multivariada com média $\mathbf{X}_{i} \boldsymbol{\beta}$ e matriz de variâncias e covariâncias $\sigma^{2} \boldsymbol{\Sigma}_{i}$.

A estimação dos parâmetros do modelo pode ser realizada por Máxima Verossimilhança e Máxima Verossimilhança Restrita. 


\section{Máxima Verossimilhança}

Considere o modelo apresentado em (4.33). Os parâmetros do modelo são $\boldsymbol{\beta}, \sigma^{2}$ e $\boldsymbol{\theta}$ o qual representa os parâmetros que definem $\boldsymbol{\Sigma}_{i}$.

Pelo método de estimação por Máxima Verossimilhança temos que os estimadores são obtidos por meio da maximização da função de verossimilhança ou logverossimilhança. Sendo assim, temos que a função de verossimilhança para o modelo é dada por:

$$
L\left(\boldsymbol{\beta}, \boldsymbol{\theta}, \sigma^{2} \mid \mathbf{y}\right)=\prod_{i=1}^{M}(2 \pi)^{-\frac{n_{i}}{2}}\left(\sigma^{2}\right)^{-\frac{n_{i}}{2}}\left|\boldsymbol{\Sigma}_{i}\right|^{-\frac{1}{2}} \exp \left[-\frac{1}{2 \sigma^{2}}\left(\mathbf{y}_{i}-\mathbf{X}_{i} \boldsymbol{\beta}\right)^{T} \boldsymbol{\Sigma}_{i}^{-1}\left(\mathbf{y}_{i}-\mathbf{X}_{i} \boldsymbol{\beta}\right)\right]
$$

Podemos ainda obter a log-verossimilhança para o modelo, sendo dada por:

$$
\begin{aligned}
l\left(\boldsymbol{\beta}, \boldsymbol{\theta}, \sigma^{2} \mid \mathbf{y}\right) & =-\frac{N}{2} \log (2 \pi)-\frac{N}{2} \log \sigma^{2}+\sum_{i=1}^{M}-\frac{1}{2} \log \left(\left|\boldsymbol{\Sigma}_{i}\right|\right)- \\
& -\frac{1}{2 \sigma^{2}}\left[\sum_{i=1}^{M}\left(\mathbf{y}_{i}-\mathbf{X}_{i} \boldsymbol{\beta}\right)^{T} \mathbf{\Sigma}_{i}^{-1}\left(\mathbf{y}_{i}-\mathbf{X}_{i} \boldsymbol{\beta}\right)\right]
\end{aligned}
$$

em que $\sum_{i=1}^{M} n_{i}=N$.

Supondo $\boldsymbol{\Sigma}_{i}$ conhecida, temos que os valores de $\boldsymbol{\beta}$ e $\sigma^{2}$ que maximizam a logverossimilhança em (4.38) são obtidos por meio da resolução das equações

$$
\frac{\partial l}{\partial \boldsymbol{\beta}}=0 \Longrightarrow \sum_{i=1}^{M} \mathbf{X}_{i}^{T} \boldsymbol{\Sigma}_{i}^{-1}\left(\mathbf{y}_{i}-\mathbf{X}_{i} \boldsymbol{\beta}\right)=0
$$

$\mathrm{e}$

$$
\frac{\partial l}{\partial \sigma^{2}}=0 \Longrightarrow-\frac{N}{2 \sigma^{2}}+\frac{1}{2 \sigma^{4}} \sum_{i=1}^{M}\left(\mathbf{y}_{i}-\mathbf{X}_{i} \boldsymbol{\beta}\right)^{T} \boldsymbol{\Sigma}_{i}^{-1}\left(\mathbf{y}_{i}-\mathbf{X}_{i} \boldsymbol{\beta}\right)=0
$$

Portanto, os estimadores de $\boldsymbol{\beta}$ e $\sigma^{2}$ são dados por

$$
\begin{aligned}
\hat{\boldsymbol{\beta}}(\boldsymbol{\theta}) & =\left(\sum_{i=1}^{M} \mathbf{X}_{i}^{\mathrm{T}} \boldsymbol{\Sigma}_{i}^{-1} \mathbf{X}_{i}\right)^{-1} \mathbf{X}_{i}^{T} \boldsymbol{\Sigma}_{i}^{-1} \mathbf{y}_{i} \\
\hat{\sigma}^{2}(\boldsymbol{\theta}) & =\frac{\sum_{i=1}^{M}\left(\mathbf{y}_{i}-\mathbf{X}_{i} \hat{\boldsymbol{\beta}}(\boldsymbol{\theta})\right)^{T} \boldsymbol{\Sigma}_{i}^{-1}\left(\mathbf{y}_{i}-\mathbf{X}_{i} \hat{\boldsymbol{\beta}}(\boldsymbol{\theta})\right)}{N} .
\end{aligned}
$$

Substituindo $\hat{\sigma}^{2}(\boldsymbol{\theta})$ e $\hat{\boldsymbol{\beta}}(\boldsymbol{\theta})$ em (4.38), obtém-se a função de log-verossimilhança 
perfilada, sendo função apenas de $\boldsymbol{\theta}$ :

$$
\begin{aligned}
l(\boldsymbol{\theta} \mid \mathbf{y}) & =\frac{N}{2}\left[\log (N)-\log (2 \pi)-\log \left(\sum_{i=1}^{M} \mathbf{y}_{i}-\mathbf{X}_{i} \hat{\boldsymbol{\beta}}(\boldsymbol{\theta})\right)^{T} \boldsymbol{\Sigma}_{i}^{-1}\left(\mathbf{y}_{i}-\mathbf{X}_{i} \hat{\boldsymbol{\beta}}(\boldsymbol{\theta})\right)-1\right]- \\
& -\frac{1}{2} \sum_{i=1}^{M} \log \left(\left|\boldsymbol{\Sigma}_{i}\right|\right)
\end{aligned}
$$

A log-verossimilhança perfilada em (4.43) é maximizada com respeito a $\boldsymbol{\Sigma}_{i}$, obtendo o estimador de Máxima Verossimilhança $\hat{\boldsymbol{\Sigma}}_{i}$. Então, os estimadores de Máxima Verossimilhança $\hat{\boldsymbol{\beta}}$ e $\hat{\sigma}^{2}$ são obtidos substituindo $\boldsymbol{\Sigma}_{i}$ por $\hat{\boldsymbol{\Sigma}}_{i}$.

Agora, para predizer os efeitos aleatórios $\mathbf{u}_{i}$ utiliza-se o melhor preditor linear não viesado (BLUP, do inglês Best linear unbiased prediction) (Searle [31]). Os BLUPs dos efeitos aleatórios $\mathbf{u}_{i}$ são obtidos por meio da distribuição conjunta das respostas $\mathbf{y}_{i}$ e dos efeitos aleatórios $\mathbf{u}_{i}$.

Considerando o modelo apresentado em (4.27) em sua forma matricial e a normalidade dos erros e dos efeitos aleatórios, temos por Henderson [13] que

$$
\left[\begin{array}{c}
\mathbf{u}_{i} \\
\mathbf{y}_{i}
\end{array}\right] \sim N\left(\left[\begin{array}{c}
\mathbf{0} \\
\mathbf{X}_{i} \boldsymbol{\beta}
\end{array}\right],\left[\begin{array}{cc}
\boldsymbol{\Psi} & \mathbf{\Psi}_{i}^{T} \\
\mathbf{\Psi} \mathbf{Z}_{i} & \sigma^{2} \boldsymbol{\Sigma}_{i}
\end{array}\right]\right)
$$

e por Searle [31],

$$
\mathbf{u}_{i} \mid \mathbf{y}_{i} \sim N\left[\mathbf{\Psi} \mathbf{Z}_{i}^{T} \boldsymbol{\Sigma}_{i}^{-1}\left(\mathbf{y}_{i}-\mathbf{X}_{i} \boldsymbol{\beta}\right), \mathbf{W}_{i}\right]
$$

em que

$$
\mathbf{W}_{i}=\left(\Psi-\Psi \mathbf{Z}_{i}^{T} \boldsymbol{\Sigma}_{i}^{-1} \boldsymbol{\Psi} \mathbf{Z}_{i}\right)^{-1}
$$

Portanto, o preditor de $\mathbf{u}_{i}, i=1, \ldots, M$, é dado pelas modas condicionais dos efeitos aleatórios, avaliadas nas estimativas de $\hat{\boldsymbol{\beta}}$,

$$
\hat{\mathbf{u}}_{i}=\Psi \mathbf{Z}_{i}^{T} \boldsymbol{\Sigma}_{i}^{-1}\left(\mathbf{y}_{i}-\mathbf{X}_{i} \hat{\boldsymbol{\beta}}\right)
$$

Na prática, $\boldsymbol{\Sigma}_{i}$ é substituída por seu estimador de Máxima Verossimilhança $\hat{\boldsymbol{\Sigma}}_{i}$.

\section{Máxima Verossimilhança Restrita}

Devido ao fato da estimação das componentes de variância por Máxima Verossimilhança não levar em consideração os graus de liberdade que estão envolvidos na estimação dos efeitos fixos do modelo foi criada a estimação por Máxima Verossimilhança Restrita (REML) com o intuito de corrigir esse problema. [10, 25]

A ideia por trás da estimação por Máxima Verossimilhança Restrita consiste em 
estimar as componentes de variância com base nos resíduos pós-estimação dos efeitos fixos. A REML se baseia em combinações lineares do vetor de respostas y escolhidas de tal forma que as combinações não contenham nenhum efeito fixo, sendo que essas combinações acabam representando os resíduos obtidos pós-estimação dos efeitos fixos.

Em Laird [19] é proposta uma metodologia Bayesiana para obtenção da função de verossimilhança restrita. Para isso é suposto que $\boldsymbol{\beta}$ possui distribuição uniforme em $\mathbb{R}^{p}$ e a função de verossimilhança é então obtida por meio da integração da $L\left(\boldsymbol{\beta}, \sigma^{2}, \boldsymbol{\theta}\right)$ em $\boldsymbol{\beta}$,

$$
L_{R}\left(\sigma^{2}, \boldsymbol{\theta}\right)=\int_{\boldsymbol{\beta} \in \mathbb{R}^{p}} L\left(\boldsymbol{\beta}, \sigma^{2}, \boldsymbol{\theta}\right) \mathrm{d} \boldsymbol{\beta} .
$$

Como visto anteriormente, a função de verossimilhança para o modelo (4.33) é dada por

$$
L\left(\boldsymbol{\beta}, \boldsymbol{\theta}, \sigma^{2} \mid \mathbf{y}\right)=\prod_{i=1}^{M}(2 \pi)^{-\frac{n_{i}}{2}}\left(\sigma^{2}\right)^{-\frac{n_{i}}{2}}\left|\boldsymbol{\Sigma}_{i}\right|^{-\frac{1}{2}} \exp \left[-\frac{1}{2 \sigma^{2}}\left(\mathbf{y}_{i}-\mathbf{X}_{i} \boldsymbol{\beta}\right)^{T} \boldsymbol{\Sigma}_{i}^{-1}\left(\mathbf{y}_{i}-\mathbf{X}_{i} \boldsymbol{\beta}\right)\right]
$$

Considerando o estimador BLUE para $\hat{\boldsymbol{\beta}}$ dado por

$$
\hat{\boldsymbol{\beta}}(\boldsymbol{\theta})=\left(\sum_{i=1}^{M} \mathbf{X}_{i}^{T} \boldsymbol{\Sigma}_{i}^{-1} \mathbf{X}_{i}\right)^{-1} \mathbf{X}_{i}^{T} \boldsymbol{\Sigma}_{i}^{-1} \mathbf{y}_{i}
$$

e a identidade abaixo apresentada em Demidenko, [6]

$$
\begin{aligned}
& \left(\mathbf{y}_{i}-\mathbf{X}_{i} \boldsymbol{\beta}\right)^{T} \boldsymbol{\Sigma}_{i}^{-1}\left(\mathbf{y}_{i}-\mathbf{X}_{i} \boldsymbol{\beta}\right)= \\
& \quad=\left(\mathbf{y}_{i}-\mathbf{X}_{i} \hat{\boldsymbol{\beta}}\right)^{T-1}\left(\mathbf{y}_{i}-\mathbf{X}_{i} \hat{\boldsymbol{\beta}}\right)+(\hat{\boldsymbol{\beta}}-\boldsymbol{\beta})^{T} \mathbf{X}_{i}^{T} \boldsymbol{\Sigma}_{i}^{-1} \mathbf{X}_{i}(\hat{\boldsymbol{\beta}}-\boldsymbol{\beta})
\end{aligned}
$$

tem-se que (4.49) pode ser reescrita e a função de verossimilhança restrita pode ser definida como

$$
\begin{aligned}
L\left(\boldsymbol{\beta}, \boldsymbol{\theta}, \sigma^{2} \mid \mathbf{y}\right) & =\prod_{i=1}^{M}(2 \pi)^{-\frac{n_{i}}{2}}\left(\sigma^{2}\right)^{-\frac{n_{i}}{2}}\left|\boldsymbol{\Sigma}_{i}\right|^{-\frac{1}{2}} \exp \left[-\frac{1}{2 \sigma^{2}}\left(\mathbf{y}_{i}-\mathbf{X}_{i} \hat{\boldsymbol{\beta}}\right)^{T} \boldsymbol{\Sigma}_{i}^{-1}\left(\mathbf{y}_{i}-\mathbf{X}_{i} \hat{\boldsymbol{\beta}}\right)\right] \times \\
& \times \int_{\boldsymbol{\beta} \in \mathbb{R}^{p}} \exp \left[-\frac{1}{2 \sigma^{2}}(\hat{\boldsymbol{\beta}}-\boldsymbol{\beta})^{T} \mathbf{X}_{i}^{T} \boldsymbol{\Sigma}_{i}{ }^{-1} \mathbf{X}_{i}(\hat{\boldsymbol{\beta}}-\boldsymbol{\beta})\right] \mathrm{d} \boldsymbol{\beta} .
\end{aligned}
$$

Sabendo por Demidenko [6] que

$$
\int_{z \in \mathbb{R}^{p}} \exp \left[-\frac{1}{2}(z-m)^{T} A^{-1}(z-m)\right] \mathrm{d} z=(2 \pi)^{\frac{p}{2}}|A|^{-\frac{1}{2}}
$$


para todo vetor $m_{p \times 1}$ e toda matriz $A_{p \times p}$ positiva definida, logo

$$
\begin{aligned}
L\left(\sigma^{2}, \boldsymbol{\theta} \mid \mathbf{y}\right) & =\prod_{i=1}^{M}(2 \pi)^{-\frac{n_{i}}{2}}(2 \pi)^{\frac{p}{2}}\left(\sigma^{2}\right)^{-\frac{n_{i}}{2}}\left|\boldsymbol{\Sigma}_{i}\right|^{-\frac{1}{2}}\left|\mathbf{X}_{i}^{T} \boldsymbol{\Sigma}_{i}^{-1} \mathbf{X}_{i}\right|^{-\frac{1}{2}} \times \\
& \times \exp \left[-\frac{1}{2 \sigma^{2}}\left(\mathbf{y}_{i}-\mathbf{X}_{i} \hat{\boldsymbol{\beta}}\right)^{T} \boldsymbol{\Sigma}_{i}^{-1}\left(\mathbf{y}_{i}-\mathbf{X}_{i} \hat{\boldsymbol{\beta}}\right)\right]
\end{aligned}
$$

Pode-se ainda obter a log-verossimilhança dada por

$$
\begin{aligned}
l\left(\boldsymbol{\theta}, \sigma^{2} \mid \mathbf{y}\right) & =-\frac{1}{2}\left[(N-p)\left(\log (2 \pi)+\log \left(\sigma^{2}\right)\right)+\log \left|\sum_{i=1}^{M} \mathbf{X}_{i}^{T} \boldsymbol{\Sigma}_{i}^{-1} \mathbf{X}_{i}\right|+\right. \\
& \left.+\sum_{i=1}^{M}\left(\log \left|\boldsymbol{\Sigma}_{i}\right|+\frac{1}{\sigma^{2}}\left(\mathbf{y}_{i}-\mathbf{X}_{i} \hat{\boldsymbol{\beta}}\right)^{T} \boldsymbol{\Sigma}_{i}^{-1}\left(\mathbf{y}_{i}-\mathbf{X}_{i} \hat{\boldsymbol{\beta}}\right)\right)\right]
\end{aligned}
$$

Maximizando a log-verossimilhança em função de $\sigma^{2}$, tem-se que

$$
\hat{\sigma}^{2}=\frac{\sum_{i=1}^{M}\left(\mathbf{y}_{i}-\mathbf{X}_{i} \hat{\boldsymbol{\beta}}\right)^{T} \boldsymbol{\Sigma}_{i}^{-1}\left(\mathbf{y}_{i}-\mathbf{X}_{i} \hat{\boldsymbol{\beta}}\right)}{N-p} .
$$

Substituindo $\hat{\sigma}^{2}$ em (4.55), tem-se que a função log-verossimilhança fica em função apenas de $\boldsymbol{\Sigma}_{i}$ :

$$
\begin{aligned}
l(\boldsymbol{\theta} \mid \mathbf{y}) & =-\frac{1}{2}\left[N-p \log (2 \pi)+\log \left(\sum_{i=1}^{M}\left(\mathbf{y}_{i}-\mathbf{X}_{i} \hat{\boldsymbol{\beta}}\right)^{T} \boldsymbol{\Sigma}_{i}^{-1}\left(\mathbf{y}_{i}-\mathbf{X}_{i} \hat{\boldsymbol{\beta}}\right)\right)+\right. \\
& \left.+\log \left|\sum_{i=1}^{M} \mathbf{X}_{i}^{T} \boldsymbol{\Sigma}_{i}^{-1} \mathbf{X}_{i}\right|+\sum_{i=1}^{M} \log \left|\boldsymbol{\Sigma}_{i}\right|\right]
\end{aligned}
$$

A maximização da $l(\boldsymbol{\theta} \mid \mathbf{y})$ em relação a $\boldsymbol{\Sigma}_{i}$ por meio de ferramentas computacionais proporciona a obtenção do estimador de $\boldsymbol{\Sigma}_{i}$. Com isso podemos obter os estimadores de $\boldsymbol{\beta}$ e $\sigma^{2}$ substituindo $\boldsymbol{\Sigma}_{i}$ pelo seu estimador $\hat{\boldsymbol{\Sigma}}_{i}$.

\section{Modelo Linear Misto com dois níveis}

O Modelo Linear Misto considerando dois níveis de aleatorização hierárquicos, segue a mesma estruturação que o Modelo Linear Misto com apenas um nível apresentado na seção 4.1.2.

Sendo assim, tem-se que o modelo com dois níveis pode ser representado da seguinte maneira:

$$
\mathbf{y}_{i j}=\mathbf{X}_{i j} \boldsymbol{\beta}+\mathbf{Z}_{i, j} \mathbf{u}_{i}+\mathbf{Z}_{i j} \mathbf{u}_{i j}+\boldsymbol{\epsilon}_{i j} ; \quad i=1, \ldots, M ; j=1, \ldots, M_{i},
$$


tendo

$$
\begin{aligned}
\boldsymbol{\epsilon}_{i j} & \sim N\left(\mathbf{0} ; \sigma^{2} I\right), \\
\mathbf{u}_{i} & \sim N\left(\mathbf{0} ; \boldsymbol{\Psi}_{1}\right), \\
\mathbf{u}_{i j} & \sim N\left(\mathbf{0} ; \boldsymbol{\Psi}_{2}\right),
\end{aligned}
$$

e $\boldsymbol{\Psi}_{1}$ e $\boldsymbol{\Psi}_{2}$ positivas definidas.

Supõe-se que $\mathbf{u}_{i}$ são independentes para diferentes $i, \mathbf{u}_{i j}$ são independentes para diferentes $i$ ou $j$ e independentes de $\mathbf{u}_{i}$. Os erros intra-grupo $\boldsymbol{\epsilon}_{i j}$ são independentes para diferentes $i$ ou $j$ e independentes entre os efeitos aleatórios.

Considerando os mesmos passos realizados para o Modelo Linear Misto com apenas um nível, tem-se que a distribuição marginal das respostas $\mathbf{y}_{i j}$ é dada por

$$
\mathbf{y}_{i j} \sim N\left(\mathbf{X}_{i j} \boldsymbol{\beta} ; \boldsymbol{\Sigma}_{i j}\right)
$$

em que

$$
\boldsymbol{\Sigma}_{i j}=\mathbf{I}+\frac{\mathbf{Z}_{i, j} \Psi_{1} \mathbf{Z}_{i, j}^{T}+\mathbf{Z}_{i j} \Psi_{2} \mathbf{Z}_{i j}^{T}}{\sigma^{2}}
$$

Com base na distribuição marginal de $\mathbf{y}_{i j}$ podem ser obtidos os estimadores do modelo e os preditores dos efeitos aleatórios.

Considerando primeiramente a estimação por Máxima Verossimilhança, tem-se que a função de verossimilhança para o modelo é dada por:

$$
\begin{aligned}
L\left(\boldsymbol{\beta}, \boldsymbol{\Sigma}_{i j}, \sigma^{2} \mid \mathbf{y}\right) & =\prod_{i=1}^{M} \prod_{j=1}^{M_{i}}(2 \pi)^{-\frac{n_{i j}}{2}}\left(\sigma^{2}\right)^{-\frac{n_{i j}}{2}}\left|\boldsymbol{\Sigma}_{i j}\right|^{-\frac{1}{2}} \times \\
& \times \exp \left[-\frac{1}{2 \sigma^{2}}\left(\mathbf{y}_{i j}-\mathbf{X}_{i j} \boldsymbol{\beta}\right)^{T} \boldsymbol{\Sigma}_{i j}{ }^{-1}\left(\mathbf{y}_{i j}-\mathbf{X}_{i j} \boldsymbol{\beta}\right)\right]
\end{aligned}
$$

Pode-se ainda obter a log-verossimilhança para o modelo, sendo dada por:

$$
\begin{aligned}
l\left(\boldsymbol{\beta}, \boldsymbol{\Sigma}_{i j}, \sigma^{2} \mid \mathbf{y}\right) & =-\frac{N}{2} \log (2 \pi)-\frac{N}{2} \log \sigma^{2}+\sum_{i=1}^{M} \sum_{j=1}^{M_{i}}-\frac{1}{2} \log \left(\left|\boldsymbol{\Sigma}_{i j}\right|\right)- \\
& -\frac{1}{2 \sigma^{2}}\left[\sum_{i=1}^{M} \sum_{j=1}^{M_{i}}\left(\mathbf{y}_{i j}-\mathbf{X}_{i j} \boldsymbol{\beta}\right)^{T} \boldsymbol{\Sigma}_{i j}{ }^{-1}\left(\mathbf{y}_{i j}-\mathbf{X}_{i j} \boldsymbol{\beta}\right)\right],
\end{aligned}
$$

em que, $\sum_{i=1}^{M} \sum_{j=1}^{M_{i}} n_{i j}=N$. 
Os estimadores de $\boldsymbol{\beta}$ e de $\sigma^{2}$ são dados por:

$$
\hat{\boldsymbol{\beta}}\left(\boldsymbol{\Sigma}_{i j}\right)=\left(\sum_{i=1}^{M} \sum_{j=1}^{M_{i}} \mathbf{X}_{i j}^{T} \boldsymbol{\Sigma}_{i j}{ }^{-1} \mathbf{X}_{i j}\right)^{-1} \mathbf{X}_{i j}^{T} \boldsymbol{\Sigma}_{i j}{ }^{-1} \mathbf{y}_{i j}
$$

e

$$
\hat{\sigma}^{2}\left(\boldsymbol{\Sigma}_{i j}\right)=\frac{\sum_{i=1}^{M} \sum_{j=1}^{M_{i}}\left(\mathbf{y}_{i j}-\mathbf{X}_{i j} \hat{\boldsymbol{\beta}}\right)^{T} \boldsymbol{\Sigma}_{i j}^{-1}\left(\mathbf{y}_{i j}-\mathbf{X}_{i j} \hat{\boldsymbol{\beta}}\right)}{N},
$$

sendo que, ao substituir esses resultados em (4.65), obtém-se a log-verossimilhança em função apenas de $\boldsymbol{\Sigma}_{i j}$,

$$
\begin{aligned}
& l\left(\boldsymbol{\Sigma}_{i j} \mid \mathbf{y}\right)= \\
& =\frac{N}{2}\left[\log (N)-\log (2 \pi)-\log \left(\sum_{i=1}^{M} \sum_{j=1}^{M_{i}}\left(\mathbf{y}_{i j}-\mathbf{X}_{i j} \hat{\boldsymbol{\beta}}\left(\boldsymbol{\Sigma}_{i j}\right)\right)^{\mathrm{T}} \boldsymbol{\Sigma}_{i j}{ }^{-1}\left(\mathbf{y}_{i j}-\mathbf{X}_{i j} \hat{\boldsymbol{\beta}}\left(\boldsymbol{\Sigma}_{i}\right)\right)\right)-1\right]- \\
& -\frac{1}{2} \sum_{i=1}^{M} \sum_{j=1}^{M_{i}} \log \left(\left|\boldsymbol{\Sigma}_{i j}\right|\right) .
\end{aligned}
$$

A log-verossimilhança perfilada é maximizada com respeito a $\boldsymbol{\Sigma}_{i j}$, obtendo o estimador de Máxima Verossimilhança $\hat{\boldsymbol{\Sigma}}_{i j}$. Então, os estimadores de Máxima Verossimilhança $\hat{\boldsymbol{\beta}}$ e $\hat{\sigma}^{2}$ são obtidos substituindo $\boldsymbol{\Sigma}_{i j}$ por $\hat{\boldsymbol{\Sigma}}_{i j}$.

Considerando agora a estimação dos parâmetros por Máxima Verossimilhança Restrita que corrige os graus de liberdade das componentes de variância de acordo com o número de efeitos fixos presentes no modelo, assim como foi realizado para o Modelo Linear Misto com apenas um nível, a função de verossimilhança para o modelo com dois níveis é obtida por meio da integração da verossimilhança completa em $\boldsymbol{\beta}$,

$$
L_{R}\left(\sigma^{2}, \boldsymbol{\Sigma}_{i j}\right)=\int_{\boldsymbol{\beta} \in \mathbb{R}^{p}} L\left(\boldsymbol{\beta}, \sigma^{2}, \boldsymbol{\Sigma}_{i j}\right) \mathrm{d} \boldsymbol{\beta} .
$$

Considerando o estimador BLUE para $\boldsymbol{\beta}$ e o estimador para $\sigma^{2}$ dados a seguir:

$$
\begin{gathered}
\hat{\boldsymbol{\beta}}\left(\boldsymbol{\Sigma}_{i j}\right)=\left(\sum_{i=1}^{M} \sum_{j=1}^{M_{i}} \mathbf{X}_{i j}^{T} \boldsymbol{\Sigma}_{i j}{ }^{-1} \mathbf{X}_{i j}\right)^{-1} \mathbf{X}_{i j}^{T} \boldsymbol{\Sigma}_{i j}{ }^{-1} \mathbf{y}_{i j}, \\
\hat{\sigma}^{2}\left(\boldsymbol{\Sigma}_{i j}\right)=\frac{\sum_{i=1}^{M} \sum_{j=1}^{M_{i}}\left(\mathbf{y}_{i j}-\mathbf{X}_{i j} \hat{\boldsymbol{\beta}}\right)^{T} \boldsymbol{\Sigma}_{i j}{ }^{-1}\left(\mathbf{y}_{i j}-\mathbf{X}_{i j} \hat{\boldsymbol{\beta}}\right)}{N-p},
\end{gathered}
$$

e seguindo os mesmos passos realizados anteriormente para o modelo com um único 
nível, tem-se que a log-verossimilhança somente em termos de $\boldsymbol{\Sigma}_{i j}$ é dada por:

$$
\begin{aligned}
l\left(\boldsymbol{\Sigma}_{i j} \mid \mathbf{y}\right) & =-\frac{1}{2}\left[N-p \log (2 \pi)+\log \left(\sum_{i=1}^{M} \sum_{j=1}^{M_{i}}\left(\mathbf{y}_{i j}-\mathbf{X}_{i j} \hat{\boldsymbol{\beta}}\right)^{T} \boldsymbol{\Sigma}_{i}{ }^{-1}\left(\mathbf{y}_{i j}-\mathbf{X}_{i j} \hat{\boldsymbol{\beta}}\right)\right)+\right. \\
& \left.+\log \left|\sum_{i=1}^{M} \sum_{j=1}^{M_{i}} \mathbf{X}_{i j}^{T} \boldsymbol{\Sigma}_{i j}{ }^{-1} \mathbf{X}_{i j}\right|+\sum_{i=1}^{M} \sum_{j=1}^{M_{i}} \log \left|\boldsymbol{\Sigma}_{i j}\right|\right]
\end{aligned}
$$

$l\left(\boldsymbol{\Sigma}_{i j} \mid \mathbf{y}\right)$ deve ser maximizada em termos de $\boldsymbol{\Sigma}_{i j}$ por meio de ferramentas computacionais para obtenção do estimador de $\boldsymbol{\Sigma}_{i j}$. Com o estimador $\hat{\boldsymbol{\Sigma}}_{i j}$ é possível obter os estimadores por Máxima Verossimilhança Restrita de $\boldsymbol{\beta}$ e $\sigma^{2}$, bastando substituir $\Sigma_{i j}$ por $\hat{\Sigma}_{i j}$

\section{Resíduos}

Para os Modelos Lineares Mistos, pode-se obter três tipos de fontes aleatórias:

- $\epsilon$ : erro condicional;

- $u_{i}$ : efeitos aleatórios;

- $\epsilon^{*}$ : erro marginal.

O ideal seria prever tais quantidades por meio de um resíduo que seja função de uma única fonte de variação e que seja dado por quantidades conhecidas. Quando isso é possível, diz-se que tal resíduo é puro.

Por outro lado, um resíduo que depende de outras fontes de variação além da fonte de interesse é denominado resíduo com confundimento.

Considerando que o objetivo de utilizar modelos de normalização está relacionado com a retirada de fontes de variação que não são de interesse por meio da utilização dos resíduos desse modelo, têm-se que os resíduos podem ser considerados como novas observações, sem viés e acuradas.

Dessa forma, a obtenção dos resíduos condicionais e/ou marginais são de interesse neste trabalho.

\section{Resíduos condicionais}

Considerando o modelo apresentado em (4.58), tem-se que, ao considerar o modelo em sua forma matricial, os resíduos condicionais podem ser obtidos da seguinte maneira:

$$
\hat{\boldsymbol{\epsilon}}=\mathbf{y}-\mathbf{X} \hat{\boldsymbol{\beta}}-\mathbf{Z} \hat{\mathbf{u}},
$$

sendo $\hat{\boldsymbol{\beta}}$ o EBLUE de $\boldsymbol{\beta}$ e $\hat{\mathbf{u}}$ o EBLUP de $\mathbf{u}$. 


\section{Resíduos marginais}

Agora, para obter os resíduos marginais referentes ao modelo apresentado em (4.58), basta resolver a seguinte equação:

$$
\hat{\boldsymbol{\epsilon}}^{*}=\mathbf{y}-\mathbf{X} \hat{\boldsymbol{\beta}}
$$

sendo $\hat{\boldsymbol{\beta}}$ o EBLUE de $\boldsymbol{\beta}$.

\section{Ferramenta $\mathbf{R}$ - funções lme e lmer}

Os Modelos Lineares Mistos com um e dois níveis apresentados em (4.30) e (4.58) podem ser analisados e estimados por meio do software estatístico $\mathrm{R}$ com as seguintes funções lme e lmer dos pacotes nlme e lme4 respectivamente. [3, 29]

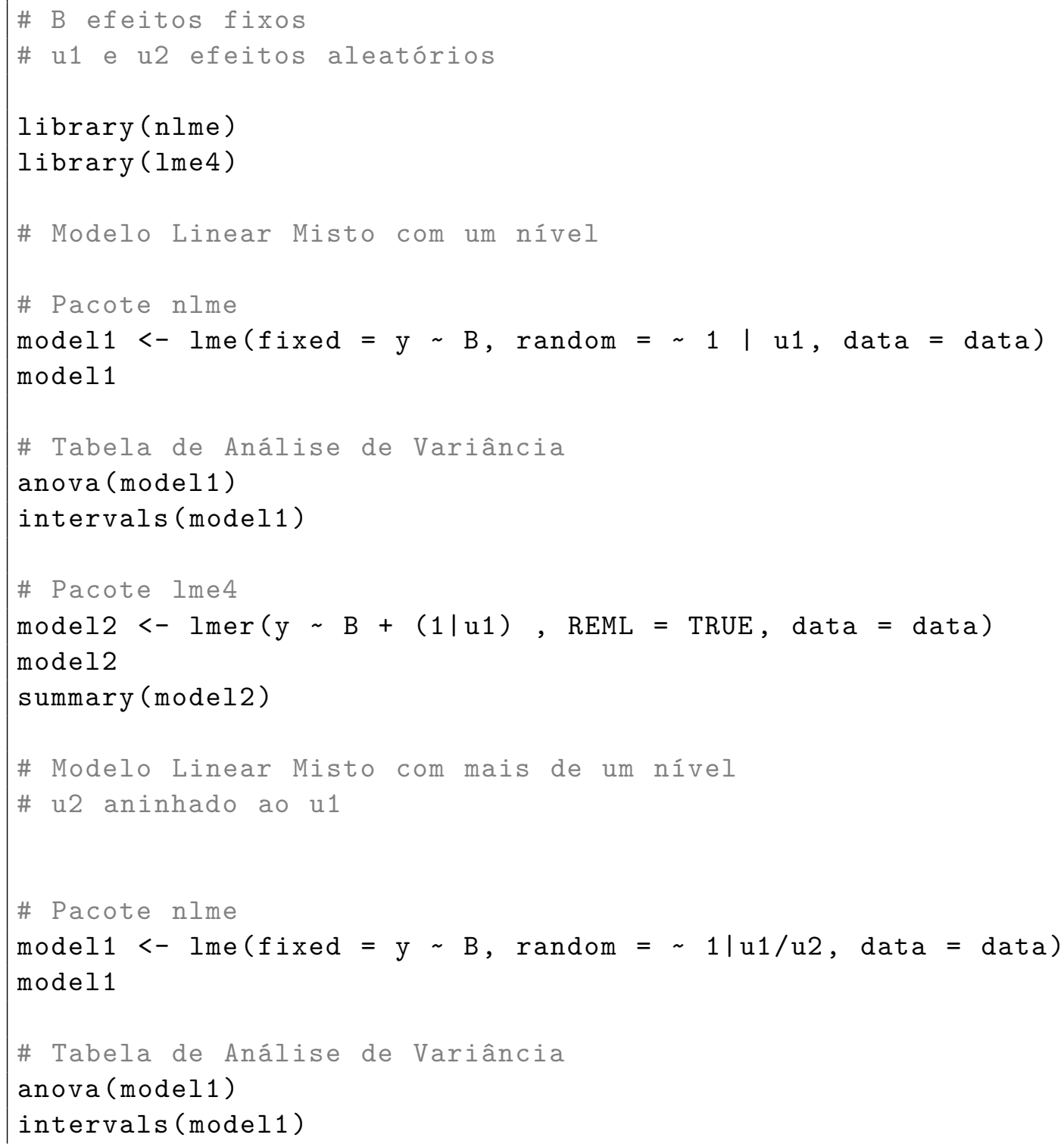




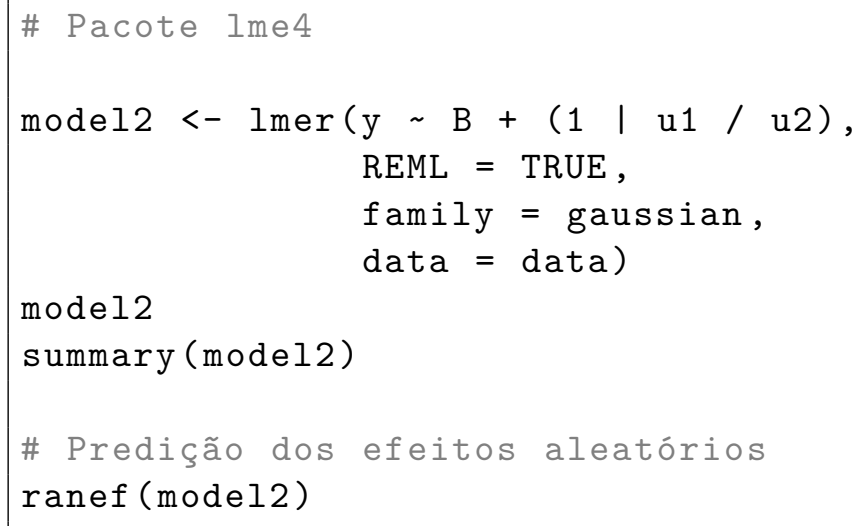

\subsubsection{Modelo Linear Misto com erros heterocedásticos}

Os modelos apresentados anteriormente restringem que o erro intragrupo $\epsilon_{i j}$ seja considerado independente e identicamente distribuído com média zero e variância constante. Nesta seção o Modelo Linear Misto com um e dois níveis será estendido para possibilitar erros heterocedásticos.

Primeiramente, considere o Modelo Linear Misto com um único nível apresentado em 4.1.2, porém sem a suposição da homocedasticidade dos erros. Pode-se definir tal modelo da seguinte maneira:

$$
\mathbf{y}_{i}=\mathbf{X}_{i} \boldsymbol{\beta}+\mathbf{Z}_{\mathbf{i}} \mathbf{u}_{i}+\boldsymbol{\epsilon}_{i} ; \quad i=1, \ldots, M
$$

na qual,

$$
\begin{aligned}
\boldsymbol{\epsilon}_{i} & \sim N\left(\mathbf{0} ; \sigma^{2} \boldsymbol{\Lambda}_{i}\right) \\
\mathbf{u}_{i} & \sim N(\mathbf{0} ; \boldsymbol{\Psi})
\end{aligned}
$$

e $\boldsymbol{\Psi}$ e $\boldsymbol{\Lambda}_{i}$ são positivas definidas.

Os erros $\boldsymbol{\epsilon}_{i}$ e os efeitos aleatórios $\mathbf{u}_{i}$ são independentes para diferentes grupos e entre si no mesmo grupo.

Por Thisted [35], como $\boldsymbol{\Lambda}_{i}$ é positiva definida pode-se obter $\boldsymbol{\Lambda}_{i}^{\frac{1}{2}}$, com inversa $\boldsymbol{\Lambda}_{i}^{-\frac{1}{2}}$. Portanto,

$$
\boldsymbol{\Lambda}_{i}=\left(\boldsymbol{\Lambda}_{i}^{\frac{1}{2}}\right)^{\mathrm{T}} \boldsymbol{\Lambda}_{i}^{\frac{1}{2}}
$$

$\mathrm{e}$

$$
\boldsymbol{\Lambda}_{i}^{-1}=\left(\boldsymbol{\Lambda}_{i}^{-\frac{1}{2}}\right)^{\mathrm{T}} \boldsymbol{\Lambda}_{i}^{-\frac{1}{2}}
$$


Define-se,

$$
\begin{aligned}
\mathbf{y}_{i}^{*} & =\left(\boldsymbol{\Lambda}_{i}^{-\frac{1}{2}}\right)^{\mathrm{T}} \mathbf{y}_{i}, \\
\boldsymbol{\epsilon}_{i}^{*} & =\left(\boldsymbol{\Lambda}_{i}^{-\frac{1}{2}}\right)^{\mathrm{T}} \boldsymbol{\epsilon}_{i}, \\
\mathbf{X}_{i}^{*} & =\left(\boldsymbol{\Lambda}_{i}^{-\frac{1}{2}}\right)^{\mathrm{T}} \mathbf{X}_{i},
\end{aligned}
$$

e

$$
\mathbf{Z}_{i}^{*}=\left(\boldsymbol{\Lambda}_{i}^{-\frac{1}{2}}\right)^{\mathrm{T}} \mathbf{Z}_{i}
$$

Considerando as transformações realizadas, temos que

$$
\boldsymbol{\epsilon}_{i}^{*} \sim N\left[\left(\boldsymbol{\Lambda}_{i}^{-\frac{1}{2}}\right)^{\mathrm{T}} \mathbf{0} ; \sigma^{2}\left(\boldsymbol{\Lambda}_{i}^{-\frac{1}{2}}\right)^{\mathrm{T}} \boldsymbol{\Lambda}_{i}\left(\boldsymbol{\Lambda}_{i}^{-\frac{1}{2}}\right)\right] .
$$

Logo,

$$
\boldsymbol{\epsilon}_{i}^{*} \sim N\left[\mathbf{0} ; \sigma^{2} I\right]
$$

Dessa forma, o modelo (4.75) pode ser reescrito como

$$
\mathbf{y}_{i}^{*}=\mathbf{X}_{i}^{*} \boldsymbol{\beta}+\mathbf{Z}_{i}^{*} \mathbf{u}_{i}+\boldsymbol{\epsilon}_{i}^{*} ; \quad i=1, \ldots, M
$$

no qual

$$
\boldsymbol{\epsilon}_{i}^{*} \sim N\left(\mathbf{0} ; \sigma^{2} I\right)
$$

e

$$
\mathbf{u}_{i} \sim N(\mathbf{0} ; \boldsymbol{\Psi})
$$

Tem-se que o modelo (4.86) é equivalente ao Modelo Linear Misto Homocedástico com apenas um nível apresentado na seção 4.1.2.

Devido à transformação $\mathbf{y}_{i}^{*}=\left(\boldsymbol{\Lambda}_{i}^{-\frac{1}{2}}\right)^{\mathrm{T}} \mathbf{y}_{i}$, tem-se que $\mathrm{d} \mathbf{y}_{i}^{*}=\left|\boldsymbol{\Lambda}_{i}^{-\frac{1}{2}}\right| \mathrm{d} \mathbf{y}_{i}$. Portanto, a função de verossimilhança $L\left(\boldsymbol{\beta}, \sigma^{2}, \lambda, \boldsymbol{\Sigma}_{i}\right)$ para o modelo é dada por,

$$
L\left(\boldsymbol{\beta}, \sigma^{2}, \lambda, \boldsymbol{\Sigma}_{i} \mid \mathbf{y}\right)=L\left(\boldsymbol{\beta}, \sigma^{2}, \lambda, \boldsymbol{\Sigma}_{i} \mid \mathbf{y}^{*}\right) \prod_{i=1}^{M}\left|\boldsymbol{\Lambda}_{i}^{-\frac{1}{2}}\right|,
$$

em que $\lambda$ representa os parâmetros que definem $\boldsymbol{\Lambda}_{i}$.

Dessa forma, $L\left(\boldsymbol{\beta}, \sigma^{2}, \lambda, \boldsymbol{\Sigma}_{i} \mid \mathbf{y}^{*}\right)$ corresponde à verossimilhança do Modelo Linear Misto Homocedástico, podendo assim considerar os resultados obtidos na seção 4.1.2 como equivalentes para o modelo apresentado em 4.86 . 
Além disso, pode-se ainda obter a função de verossimilhança restrita para o modelo, bastando integrar a verossimilhança em relação à $\boldsymbol{\beta}$, obtendo

$$
\begin{aligned}
L_{R}\left(\sigma^{2}, \boldsymbol{\Sigma}_{i}, \lambda \mid \mathbf{y}\right) & =\int_{\boldsymbol{\beta} \in \mathbb{R}^{p}} L\left(\boldsymbol{\beta}, \sigma^{2}, \boldsymbol{\Sigma}_{i}, \lambda \mid \mathbf{y}\right) \mathrm{d} \boldsymbol{\beta} \\
& =L_{R}\left(\boldsymbol{\beta}, \sigma^{2}, \boldsymbol{\Sigma}_{i}, \lambda \mid \mathbf{y}^{*}\right) \prod_{i=1}^{M}\left|\boldsymbol{\Lambda}_{i}^{-\frac{1}{2}}\right|
\end{aligned}
$$

sendo que, $L_{R}\left(\boldsymbol{\beta}, \sigma^{2}, \boldsymbol{\Sigma}_{i}, \lambda \mid \mathbf{y}^{*}\right)$ equivale à verossimilhança restrita obtida para o Modelo Linear Misto Homocedástico na seção 4.1.2.

Analogamente, pode-se obter as funções de verossimilhança para o caso em que se tem mais de um nível no modelo.

Sendo assim, a função de verossimilhança para o Modelo Linear Misto com dois níveis e erros heterocedásticos é dada por:

$$
L\left(\boldsymbol{\beta}, \sigma^{2}, \lambda, \boldsymbol{\Sigma}_{i j} \mid \mathbf{y}\right)=L\left(\boldsymbol{\beta}, \sigma^{2}, \lambda, \boldsymbol{\Sigma}_{i j} \mid \mathbf{y}^{*}\right) \prod_{i=1}^{M} \prod_{j=1}^{M_{i}}\left|\boldsymbol{\Lambda}_{i j}^{-\frac{1}{2}}\right|
$$

em que, $L\left(\boldsymbol{\beta}, \sigma^{2}, \lambda, \boldsymbol{\Sigma}_{i j} \mid \mathbf{y}^{*}\right)$ corresponde à verossimilhança do Modelo Linear Misto Homocedástico com dois níveis.

Assim, pode-se obter a função de verossimilhança restrita para o modelo, sendo dada por

$$
\begin{aligned}
L_{R}\left(\sigma^{2}, \boldsymbol{\Sigma}_{i j}, \lambda \mid \mathbf{y}\right) & =\int_{\boldsymbol{\beta} \in \mathbb{R}^{p}} L\left(\boldsymbol{\beta}, \sigma^{2}, \boldsymbol{\Sigma}_{i j}, \lambda \mid \mathbf{y}\right) \mathrm{d} \boldsymbol{\beta} \\
& =L_{R}\left(\boldsymbol{\beta}, \sigma^{2}, \boldsymbol{\Sigma}_{i j}, \lambda \mid \mathbf{y}^{*}\right) \prod_{i=1}^{M} \prod_{j=1}^{M_{i}}\left|\boldsymbol{\Lambda}_{i j}^{-\frac{1}{2}}\right|
\end{aligned}
$$

Portanto, pode-se considerar os resultados obtidos para o Modelo Homocedástico, porém considerando o termo $\left|\boldsymbol{\Lambda}_{i j}^{-\frac{1}{2}}\right|$ a mais, sendo que, $\boldsymbol{\Lambda}_{i j}$ deve ser estimada, assim como $\boldsymbol{\Sigma}_{i j}$, por meio de ferramentas computacionais.

\section{Resíduos condicionais}

Os resíduos condicionais para o modelo heterocedástico podem ser obtidos seguindo a mesma lógica que os do modelo homocedástico, sendo possível obter os resíduos condicionais a partir da equação a seguir:

$$
\hat{\boldsymbol{\epsilon}}=\mathbf{y}-\mathbf{X} \hat{\boldsymbol{\beta}}-\mathbf{Z} \hat{\mathbf{u}},
$$


em que $\hat{\boldsymbol{\beta}}$ é o EBLUE de $\boldsymbol{\beta}$ e $\hat{\mathbf{u}}$ é o EBLUP de $\mathbf{u}$.

\section{Resíduos marginais}

A obtenção dos resíduos marginais do modelo heterocedástico é dada pela solução da seguinte equação:

$$
\hat{\boldsymbol{\epsilon}}^{*}=\mathbf{y}-\mathbf{X} \hat{\boldsymbol{\beta}}
$$

sendo $\hat{\boldsymbol{\beta}}$ o EBLUE de $\boldsymbol{\beta}$.

\section{Ferramenta R - função lme}

Os Modelos Lineares Mistos Heterocedásticos com um e mais níveis podem ser estimados e avaliados por meio da função lme do pacote nlme.

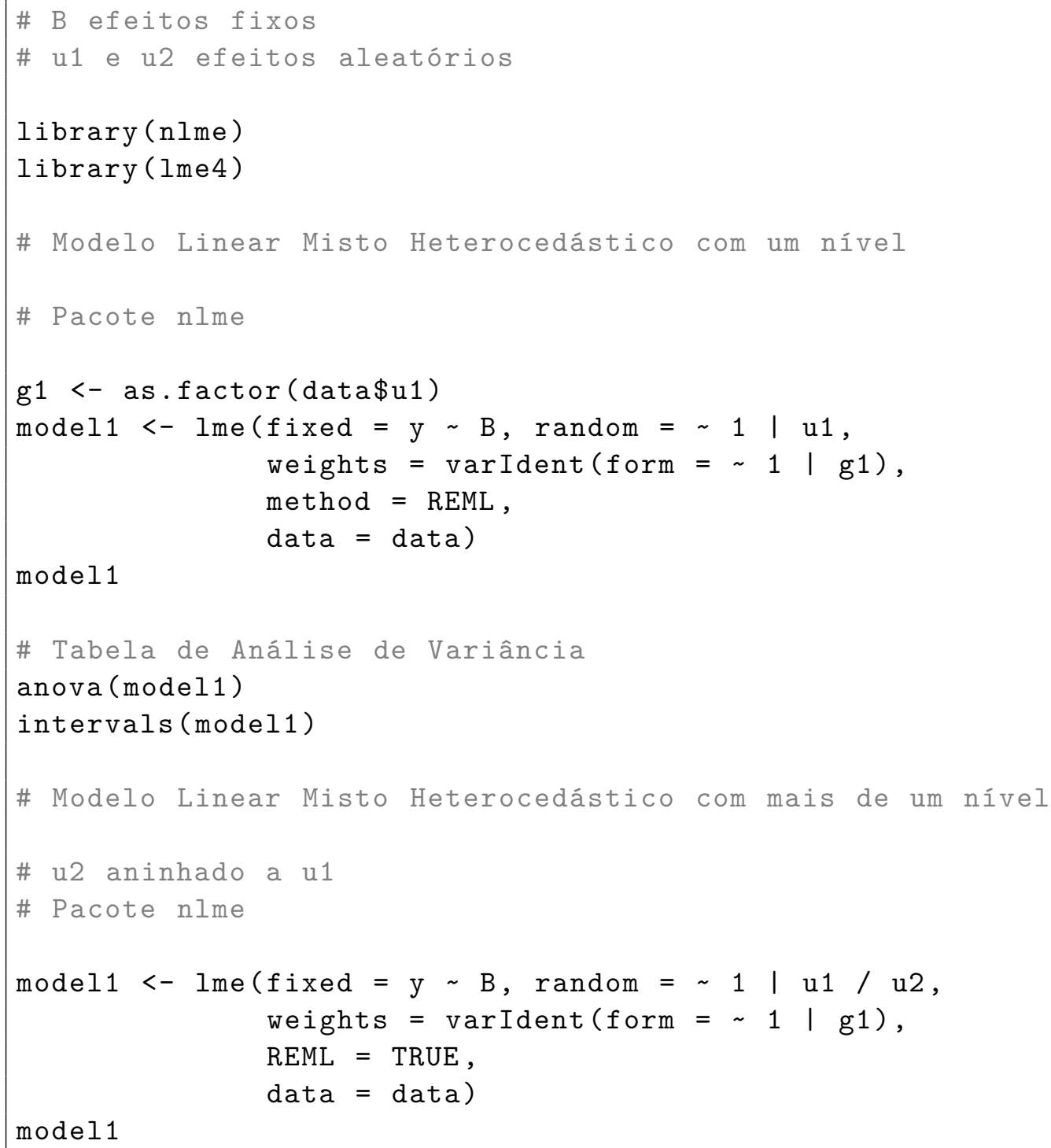




\subsection{Modelo de significância}

Um dos objetivos principais em estudos proteômicos consiste em detectar mudanças significantes na abundância de proteínas de acordo com diferentes condições que estão sendo avaliadas. A análise de significância nesses estudos é comumente realizada por meio de Testes $t$ em que são comparadas as médias das abundâncias das proteínas entre pares de condições de interesse, em geral, seguidos com correções para múltiplos testes no valor de $p$, o nível descritivo global. Todavia, é comum ter um número pequeno de amostras, o que pode gerar imprecisão nas estimativas da variabilidade amostral, influenciando na detecção das proteínas com verdadeira expressão diferencial. Muitas vezes proteínas com um alto valor de fold-change, ou seja, que apresentam uma grande variação entre diferentes condições (ex. controle e tratamento) são consideradas não significantes. Enquanto, proteínas com baixo foldchange são consideradas significantes por apresentarem baixa variabilidade amostral.

Neste trabalho é proposto a utilização de um modelo linear heterocedástico, considerando os resíduos estimados pelo modelo de normalização apresentado na seção 4.1, para avaliar a significância das variáveis (proteínas e peptídeos) na comparação de grupos de interesse.

Dessa forma, para cada variável (peptídeo e proteína) será estimado o modelo a seguir e, por meio dos resultados obtidos, serão realizadas comparações múltiplas com correção da taxa de falsos positivos pelo método FDR [34] dos fatores de interesse (método dentro de tecido) para identificação das variáveis sensíveis aos diferentes métodos de preparação de amostras utilizados. A visualização dos resultados será realizada por meio dos gráficos de vulcão [20], possibilitando a identificação de variáveis com alto valor de fold-change e significantes estatisticamente.

A análise de significância será avaliada por meio da estimação do seguinte modelo linear heterocedástico.

$$
\hat{r}_{i j k l}=\mu_{l}+M_{j}+M(T)_{j(i)}+\epsilon_{i j k l},
$$

em que,

$$
\epsilon_{i j k l} \sim N_{1}\left(0 ; \sigma_{i j l}^{2}\right) .
$$

Nesta análise os resíduos condicionais obtidos pelo modelo de normalização, referentes a cada variável (peptídeo ou proteína), são modelados separadamente, com o intuito de identificar os peptídeos e proteínas com comportamento diferencial biológico e estatístico ao se avaliar os diferentes métodos de preparação de amostras estudados.

\subsection{Racional da Análise}

Considerando a metodologia apresentada temos a seguir o Racional da Análise para cada recorte dos dados fornecido pelo método de espectrometria de massa. Este 
trabalho tem como objetivo geral comparar os diferentes métodos de preparação de amostras em busca de identificar o mais preciso. Os modelos de normalização a serem ajustados já partirão da suposição de presença de efeitos aleatórios, uma vez que o experimento foi construído seguindo uma estrutura Split-Split-Plot, em que as observações avaliadas são correlacionadas de forma hierárquica entre si.

1. Ajustar o modelo de normalização M2: Modelo Linear Misto homocedástico.

2. Ajustar o modelo de normalização M3: Modelo Linear Misto heterocedástico devido ao efeito do tecido.

3. Ajustar o modelo de normalização M4: Modelo Linear Misto heterocedástico devido ao efeito do método dentro do tecido.

4. Comparar a performance dos modelos ajustados e escolher o "melhor" ajuste.

5. Ajustar o modelo de significância para cada variável considerando os resíduos do modelo de normalização.

6. Realizar comparações múltiplas com controle de falsos positivos (FDR) para identificar proteínas e peptídeos com comportamento diferencial entre os fatores sob estudo e visualizar os resultados por meio dos gráficos vulcão.

Além disso, serão comparados os resultados obtidos pela metodologia de normalização proposta neste trabalho com os resultados da metodologia TIC.

\subsection{Análises Multivariadas}

É importante ressaltar que a Análise de Normalização é realizada considerando todas as proteínas (ou peptídeos) quantificadas simultaneamente em cada ajuste. Isto tem sido recomendado (ver, por exemplo, Wolfinger et al. [38]) para finalidade de tornar comparáveis as quantificações das variáveis (proteínas, bem como peptídeos). Já a Análise de Significância é realizada para os dados de cada variável (proteína e peptídeo) separadamente. No entanto, em estudos ômicos, em particular os baseados em espectrometria de massa, é comum o interesse em trabalhar com os dados de forma a estruturar as quantificações das proteínas (ou peptídeos) como respostas multivariadas, avaliadas de acordo com o delineamento sob estudo.

Neste contexto, o trabalho desenvolvido por Smilde et al. [32] permite decompor a matriz de dados multivariados em componentes que respeitam as fontes de variação adotadas no delineamento experimental. O método proposto, denominado ASCA (Anova-Simultaneous Component Analysis), permite realizar análises multivariadas, como Componentes Principais, em partições específicas da matriz original de dados multivariados. Essa teoria, devotada à matriz retangular de dados multivariados, segue um paralelo com a decomposição imposta às Somas de Quadrados e Produtos Cruzados da MANOVA, equivalentemente, às Somas de Quadrados Total em uma ANOVA, dividindo as variações e covariações em componentes ortogonais e independentes. 
Deste modo, por meio da ASCA os autores introduziram uma estratégia flexível e criaram algoritmos para decomposição dos dados multivariados em componentes ortogonais, sendo possível assim estudar efeitos específicos de interesse e comparar as variáveis coletadas de forma simultânea.

Aplicando esta teoria ao experimento em proteômica analisado neste trabalho, temos que a ASCA pode ser utilizada para decompor os dados nas componentes de variação relacionadas aos efeitos de tecido e de método aninhado ao tecido.

Para isso, em cada experimento MS, serão consideradas como variáveis respostas multivariadas as quantificações não correlacionadas e homocedásticas que passaram pelos modelos de normalização e de significância.

Neste estudo é de interesse avaliar a matriz correspondente ao efeito do método dentro do tecido, denotada por $\mathbf{M}(\mathbf{T})_{80 \times F}$, sendo $F$ o número de proteínas e peptídeos (features) quantificadas. Considerando essa componente dos dados totais, serão avaliadas duas técnicas multivariadas, quais sejam Análise de Componentes Principais e Grafos Probabilísticos para representação e entendimento do efeito do método dentro do tecido.

$$
\begin{gathered}
y_{f t m r}=\bar{y}_{f}+\left(\bar{y}_{f t}-\bar{y}_{f}\right)+\left(\bar{y}_{f t m}-\bar{y}_{f t}\right)+\left(y_{f t m r}-\bar{y}_{f t m}\right), \\
\mathbf{Y}_{t m r}=\overline{\mathbf{Y}}+\left(\overline{\mathbf{Y}}_{t}-\overline{\mathbf{Y}}\right)+\left(\overline{\mathbf{Y}}_{t m}-\overline{\mathbf{Y}}_{t}\right)+\left(\mathbf{Y}_{t m r}-\overline{\mathbf{Y}}_{t m}\right), \\
\mathbf{Y}_{80 \times F}=\mathbf{M}_{80 \times F}+\mathbf{T}_{80 \times F}+\mathbf{M}(\mathbf{T})_{80 \times F}+\mathbf{E}_{80 \times F},
\end{gathered}
$$

\subsubsection{Análise de Componentes Principais}

Considerando a decomposição dos dados realizada pela ASCA é possível avaliar por meio da utilização de Componentes Principais e do correspondente Biplot, tanto a representação do efeito de método dentro do tecido (definido em termos das linhas da matriz de dados) quanto dos peptídeos ou proteínas quantificados (variáveis nas colunas da matriz). Dessa forma, podem ser identificadas observações atípicas e até mesmo agrupamento nos dados.

A Análise de Componentes Principais (ACP) é uma técnica multivariada, que tem como objetivo explicar a variabilidade de um conjunto de variáveis com menor perda de informação possível por meio da utilização de combinações lineares dessas variáveis. Essas combinações são denominadas componentes, sendo não correlacionadas entre si e são estimadas por grau de maior explicação, ou seja, a primeira componente é a que possui a maior proporção da variabilidade explicada em relação a variação total presente nos dados, seguida da segunda com maior grau e assim por diante.

Considere $\mathbf{X}_{1}, \ldots, \mathbf{X}_{p}$ em $\mathbb{R}^{n}$, um conjunto de $p$ variáveis aleatórias sendo avaliadas em $n$ unidades amostrais. Em nossa aplicação, exceto o experimento SRM, $n$ é muito menor que $p$ tratando-se portanto de dados de alta dimensão (big-p). Neste caso, há diferentes alternativas para se obter os componentes principais, por exemplo, via soluções esparsas [40]. De maneira geral, restringe-se à obtenção das duas primeiras componentes principais, que possibilitam visualizar a estrutura dos dados por meio de gráficos bidimensionais. 
Algebricamente as componentes principais representam um novo sistema de coordenadas ortogonal e não correlacionado. As novas coordenadas representam as direções em que a variabilidade é máxima, sendo possível obter uma estrutura de covariâncias e variâncias mais simples e parcimoniosa. A obtenção das componentes principais pode ser realizada por meio da utilização da matriz de covariâncias ou de correlações das variáveis originais. Suponha que $\mathbf{X}^{\prime}=\left[\mathbf{X}_{1}, \ldots, \mathbf{X}_{p}\right]$ possui a matriz de covariâncias $\boldsymbol{\Sigma}$ com autovalores $\lambda_{1} \geq \lambda_{2} \geq \cdots \geq \lambda_{p} \geq 0$. Sendo que para cada autovalor, existe um autovetor $\mathbf{a}_{1}, \ldots, \mathbf{a}_{p}$ associado.

Utilizando os autovetores é possível obter as seguintes combinações lineares, conhecidas como componentes principais:

$$
\begin{aligned}
\mathbf{Y}_{1} & =\mathbf{a}_{1}^{\prime} \mathbf{X}=a_{11} \mathbf{X}_{1}+\cdots+a_{1 p} \mathbf{X}_{p} \\
\mathbf{Y}_{2} & =\mathbf{a}_{2}^{\prime} \mathbf{X}=a_{21} \mathbf{X}_{1}+\cdots+a_{2 p} \mathbf{X}_{p} \\
\vdots & \\
\mathbf{Y}_{p} & =\mathbf{a}_{p}^{\prime} \mathbf{X}=a_{p 1} \mathbf{X}_{1}+\cdots+a_{p p} \mathbf{X}_{p}
\end{aligned}
$$

Sendo que,

$$
\begin{aligned}
\operatorname{Var}\left(\mathbf{Y}_{i}\right) & =\mathbf{a}_{i}^{\prime} \boldsymbol{\Sigma} \mathbf{a}_{i}, \quad i=1, \ldots, p \\
\operatorname{Cov}\left(\mathbf{Y}_{i}, \mathbf{Y}_{k}\right) & =\mathbf{a}_{i}^{\prime} \boldsymbol{\Sigma} \mathbf{a}_{k}, \quad i \neq k, \quad i, k=1, \ldots, p
\end{aligned}
$$

Pode ser estabelecida equivalência entre ACP e Análise de Coordenadas Principais, ACoP, ou Escalonamento Multidimensional [7]. Este resultado é bastante importante e útil na redução de dimensionalidade em big-data, como uma alternativa inicial às soluções esparsas. Se $n \ll p$ é mais conveniente obter as Coordenadas Principais cuja decomposição espectral envolvida está baseada na forma quadrática de dimensão $n$, isto é na matriz de distâncias Euclidianas entre as observações. Se $n \gg p$ é mais conveniente obter os Componentes Principais, já que estão baseados na decomposição espectral da forma quadrática na dimensão $p$. Esses dois caminhos conduzem a soluções equivalentes. Outras informações sobre a Análise de Componentes Principais podem ser encontradas em Johnson \& Wichern. [15]

\subsubsection{Análise de Grafos Probabilísticos}

Em estudos ômicos é comum ser de interesse avaliar as relações existentes entre fenótipos, proteínas e peptídeos estudados para entender melhor os fatores associados às doenças e para auxiliar na identificação de possíveis tratamentos. Durante muito tempo esse tipo de análise era realizado pela estimação da correlação de Pearson entre as variáveis, identificando associações e não causalidades.

Portanto, foram desenvolvidos métodos probabilísticos para estimar as classes de equivalência da estrutura direcionada entre as variáveis, possibilitando a identificação de possíveis fatores associados às doenças. No qual, a utilização de estudos experimentais controlados e aleatoriezados é considerado o padrão ouro para inferir causalidade entre variáveis.

Dessa forma, este trabalho tem como objetivo avaliar de forma descritiva a estimação da estrutura de causalidade entre as variáveis estudadas, com foco na utilização 
de grafos acíclicos direcionados (DAG) considerando os resultados dos experimentos descritos no Capítulo 3, especificamente entre as proteínas, bem como entre os peptídeos.

As definições e conceitos referentes as suposições dos modelos para análise de grafos probabilisticos podem ser encontrados em Spirtes et al. [33] e Pearl [26].

A estimação das classes de equivalência é muitas vezes dada pela utilização de algoritmos automatizados. O algoritmo de PC (Peter-Clark) foi um dos primeiros algoritmos desenvolvidos, bastante utilizado por ser capaz de inferir a estrutura causal em um grande volume de dados esparsos, porém possui como suposição a suficiência causal, que nem sempre é satisfeita. Este trabalho, para efeito de exemplificação e por conveniência, utilizará o algoritmo PC para avaliar a causalidade entre as proteínas e os peptídeos quantificados e identificados nos experimentos. No entanto, existem diversos algoritmos que relaxam a suposição de suficiência causal e que podem ser utilizados para avaliar causalidade em estudos proteômicos.

O algoritmo PC inicia com um grafo completamente não direcionado. Então, todos os pares de variáveis são testados para avaliar independência marginal, caso sejam identificados como marginalmente independentes, a aresta entre eles é removida. Após esse primeiro estágio, todos os pares adjacentes restantes são escolhidos e o algoritmo realiza uma busca por um conjunto em que o par seja condicionalmente independente, caso exista, a aresta que conecta o par de variáveis é removida. Após passar por todos os pares de variáveis, o algoritmo tem como resultado o esqueleto do grafo, porém ainda não direcionado. Sendo que o próximo passo consiste em orientar o esqueleto obtido, para isso o algoritmo tenta identificar a orientação do grafo sem criar ciclos ou colisores. Muitas vezes o resultado do algoritmo é um grafo direcionado acíclico parcialmente completo (CPDAG).

Além disso, se o grafo verdadeiro não for esparso, o tempo de processamento é exponencial, chegando a $p^{k+2}$, sendo $p$ o número de vértices e $k$ o grau máximo associado a um vértice.

Vale ressaltar ainda a necessidade de considerar correção para múltiplos testes, pois para identificar a estrutura de causalidade entre variáveis, é necessário a realização de múltiplos testes que acabam aumentando a probabilidade de ocorrência do erro tipo I.

Mais detalhes sobre modelos de grafos probabilísticos podem ser encontrados em Spirtes et al. [33] e Pearl \& Verma [27]. 


\section{Capítulo 5}

\section{Análise}

As metodologias apresentadas no Capítulo 4 serão aplicadas aos experimentos SRM e DDA, com o intuito de comparar os diferentes métodos de preparação de amostras por meio da utilização dos modelos de normalização e significância propostos neste trabalho. Os modelos de normalização ajustados serão comparados em termos da estimação dos efeitos fixos relacionados ao método de digestão e a acurácia de suas estimativas. Além disso, será estimado o modelo de significância para cada peptídeo e proteína com base nos resíduos obtidos pelo modelo de normalização escolhido. A identificação dos peptídeos e proteínas significantes será dada pelos gráficos de vulcão apresentando os níveis descritivos e estimativas dos contrastes entre os métodos dentro de tecido. Por fim, serão comparados os resultados do modelo de significância ao se utilizar a técnica de normalização proposta neste trabalho com os obtidos por meio da utilização da técnica TIC utilizada na Bioinformática.

\subsection{Experimento SRM}

O experimento SRM consistiu em identificar e quantificar 64 peptídeos previamente estabelecidos em cada uma das cinco amostras dadas para cada combinação de tecido (NP, P, T, e Pool) e método de preparação (IGD, ISD, OFD e OPD). Dentre os 64 peptídeos 46 foram identificados e quantificados em todas as amostras avaliadas. Para comparar os métodos de preparação utilizados no experimento serão ajustados os modelos de normalização e significância propostos neste trabalho e pela Tabela 5.1 é possível observar parte dos dados gerados pelo experimento SRM.

A lista com os 64 peptídeos quantificados no experimento SRM está apresentada na Tabela 5.2.

Nas Figuras 5.1, 5.2 e 5.3 estão apresentados alguns gráficos descritivos referentes aos dados obtidos pelo experimento SRM. O primeiro gráfico faz referência à distribuição da abundância de peptídeos na escala logarítmica para cada um dos peptídeos quantificados, sendo possível perceber heterocedasticidade quanto à variabilidade $\mathrm{e}$ diferença na medida central para cada peptídeo.

Nas demais figuras estão apresentadas as distribuições das quantificações dos peptídeos separadas pelos efeitos de método dentro de tecido e os perfis na escala original e logarítmica, podendo notar que o método IGD, de modo geral, possui os menores valores quanto à abundância de peptídeos. 
Tabela 5.1 - Estrutura dos dados SRM

\begin{tabular}{llllr}
\hline Tecido & Método & Réplica & Peptídeo & $y$ \\
\hline NP & ISD & 1 & IPVGPETLGR & 19,65 \\
NP & IGD & 1 & IPVGPETLGR & 11,55 \\
NP & OPD & 1 & IPVGPETLGR & 33,56 \\
NP & OFD & 1 & IPVGPETLGR & 42,52 \\
$\vdots$ & $\vdots$ & $\vdots$ & $\vdots$ & $\vdots$ \\
$\mathrm{P}$ & ISD & 2 & IIDAALR & 27,70 \\
$\mathrm{P}$ & IGD & 2 & IIDAALR & 1,14 \\
$\mathrm{P}$ & OPD & 2 & IIDAALR & 22,90 \\
$\mathrm{P}$ & OFD & 2 & IIDAALR & 32,56 \\
$\vdots$ & $\vdots$ & $\vdots$ & $\vdots$ & $\vdots$ \\
$\mathrm{T}$ & ISD & 3 & VVDLLAPYAK & 47,31 \\
$\mathrm{~T}$ & IGD & 3 & VVDLLAPYAK & 14,40 \\
$\mathrm{~T}$ & OPD & 3 & VVDLLAPYAK & 44,18 \\
$\mathrm{~T}$ & OFD & 3 & VVDLLAPYAK & 55,93 \\
$\vdots$ & $\vdots$ & $\vdots$ & $\vdots$ & $\vdots$ \\
Pool & IGD & 1 & ADLIAYLK & 6,43 \\
Pool & ISD & 1 & ADLIAYLK & 37,64 \\
Pool & OFD & 1 & ADLIAYLK & 49,11 \\
Pool & OPD & 1 & ADLIAYLK & 32,39 \\
\hline & & & &
\end{tabular}

Figura 5.1 - SRM - Quantificação de Peptídeos

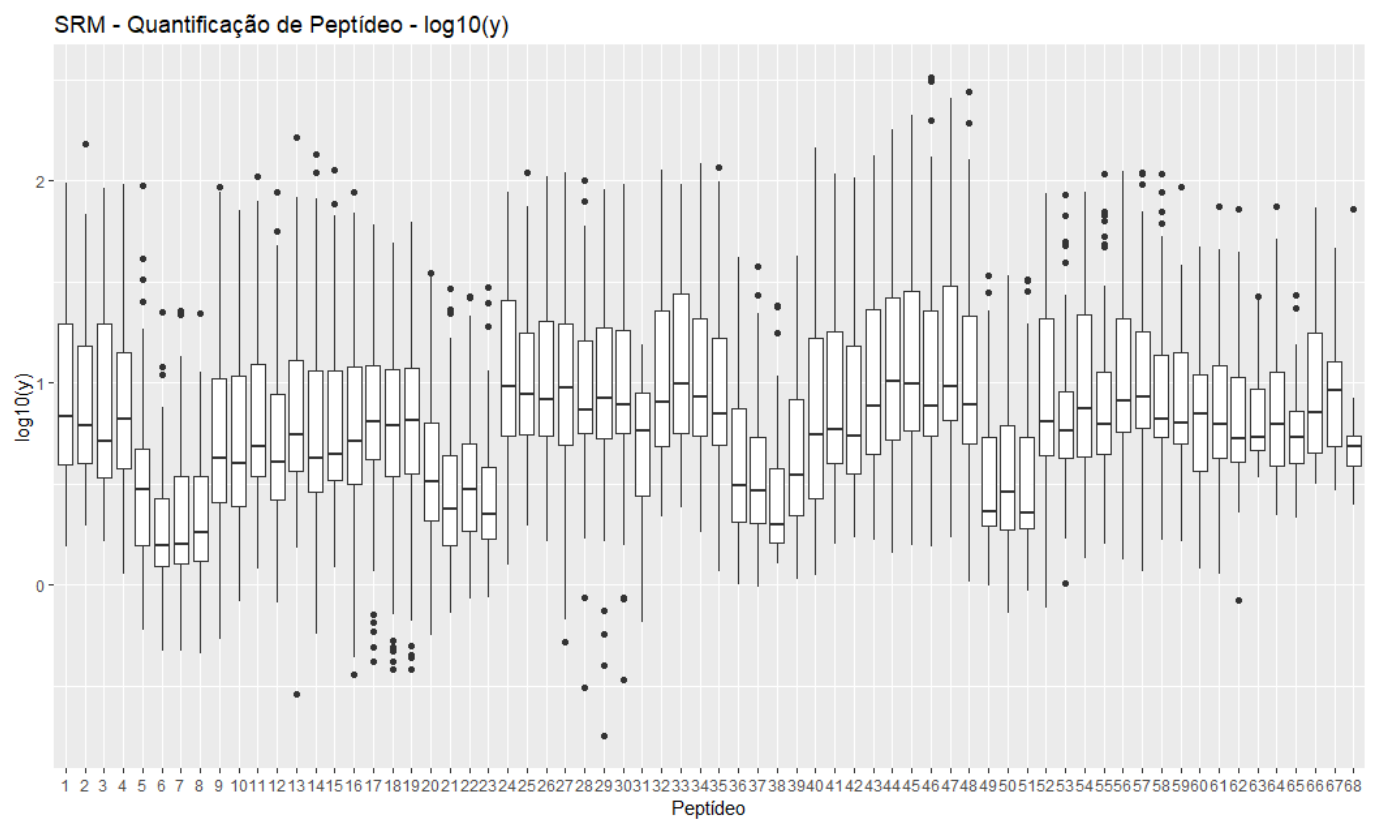


Tabela 5.2 - Lista de Peptídeos

\begin{tabular}{|c|c|c|c|}
\hline \# & Peptídeo & \# & Peptídeo \\
\hline 1 & IPVGPETLGR $^{1}$ & 2 & VVDLLAPYAK $^{1}$ \\
\hline 3 & IIDAALR $^{1}$ & 4 & ADLIAYLK ${ }^{1}$ \\
\hline 5 & TGPNLHGLFGR $^{1}$ & 6 & EFAAGYNVK $^{1}$ \\
\hline 7 & YAECGLR & 8 & TISQHQISTSIITSTQK $^{1}$ \\
\hline 9 & FASEIAGVDDLGTTGR $^{1}$ & 10 & GLLTYTSWEDALSR ${ }^{1}$ \\
\hline 11 & DFTPVCTTELGR $^{1}$ & 12 & GEGGILINSQGER $^{1}$ \\
\hline 13 & LGANSLLDLVVFGR $^{1}$ & 14 & DLVPDLSNFYAQYK $^{1}$ \\
\hline 15 & FGFYEVFK $^{1}$ & 16 & EQGFLSFWR \\
\hline 17 & AAYFGIYDTAK $^{1}$ & 18 & AIWNVINWENVTER $^{1}$ \\
\hline 19 & MVAQEGPTAFYK & 20 & NALANPLYCPDYR $^{1}$ \\
\hline 21 & NAVTQEFGPVPDTAR $^{1}$ & 22 & VAGILTVK $^{1}$ \\
\hline 23 & LVAQLYK $^{1}$ & 24 & ILVPEGTR $^{1}$ \\
\hline 25 & VPEANSSWMDTVIR $^{1}$ & 26 & ALTGGIAHLFK \\
\hline 27 & GLVVPVIR $^{1}$ & 28 & IYELAAGGTAVGTGLNTR $^{1}$ \\
\hline 29 & SGLGELILPENEPGSSIMPGK & 30 & LNEHFLNTTDFLDTIK $^{1}$ \\
\hline 31 & TIEAEAAHGTVTR & 32 & TPYTDVNIVTIR $^{1}$ \\
\hline 33 & VAVLGASGGIGQPLSLLLK $^{1}$ & 34 & VNVPVIGGHAGK $^{1}$ \\
\hline 35 & AHGFTFTR & 36 & LPCIFICENNR \\
\hline 37 & GLSSLLYGSIPK $^{1}$ & 38 & GTYQGLTATVLK $^{1}$ \\
\hline 39 & AVVVNAAQLASYSQSK $^{1}$ & 40 & GIYTGLSAGLLR $^{1}$ \\
\hline 41 & ALIADSGLK $^{1}$ & 42 & IVFSPEEAK $^{1}$ \\
\hline 43 & LIGPNCPGVINPGECK $^{1}$ & 44 & IDATQVEVNPFGETPEGQVVCFDAK ${ }^{1}$ \\
\hline 45 & VPLVVR & 46 & DFTATDLSEFAAK $^{1}$ \\
\hline 47 & FFEEVNDPAK $^{1}$ & 48 & ATAEQISSQTGNK $^{1}$ \\
\hline 49 & VAFITGGGTGLGK $^{1}$ & 50 & LLYDLADQLHAAVGASR $^{1}$ \\
\hline 51 & TIYAGNALCTVK $^{1}$ & 52 & LGPLQVAR $^{1}$ \\
\hline 53 & LSVISVEDPPQR $^{1}$ & 54 & LGAGYPMGPFELLDYVGLDTTK \\
\hline 55 & LVEVIK & 56 & DGPGFYTTR $^{1}$ \\
\hline 57 & DQLLLGPTYATPK ${ }^{1}$ & 58 & LAAAFAVSR \\
\hline 59 & QGTFHSQQALEYGTK & 60 & ITEIYEGTSEIQR \\
\hline 61 & QYGQTVATYESCSTAAFK & 62 & SEYNDQLTR \\
\hline 63 & LQINAQNCVHCK & 64 & TVEALYK \\
\hline 65 & LSVTATR & 66 & ALGVLAQLIWSR \\
\hline 67 & EISLLPDNLLR & 68 & ISAQDAK \\
\hline
\end{tabular}

${ }^{1}$ Peptídeo identificado em todas as amostras. 
Figura 5.2 - SRM - Quantificação de Peptídeos considerando Método(Tecido)
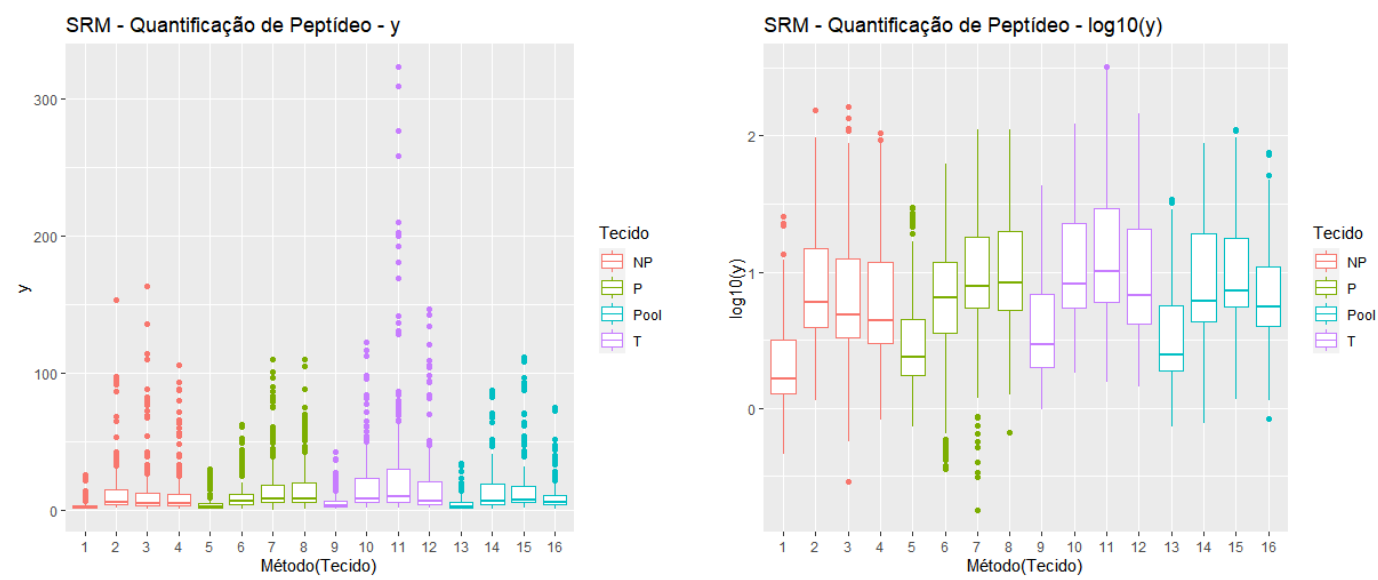

Figura 5.3 - SRM - Gráfico de Perfis
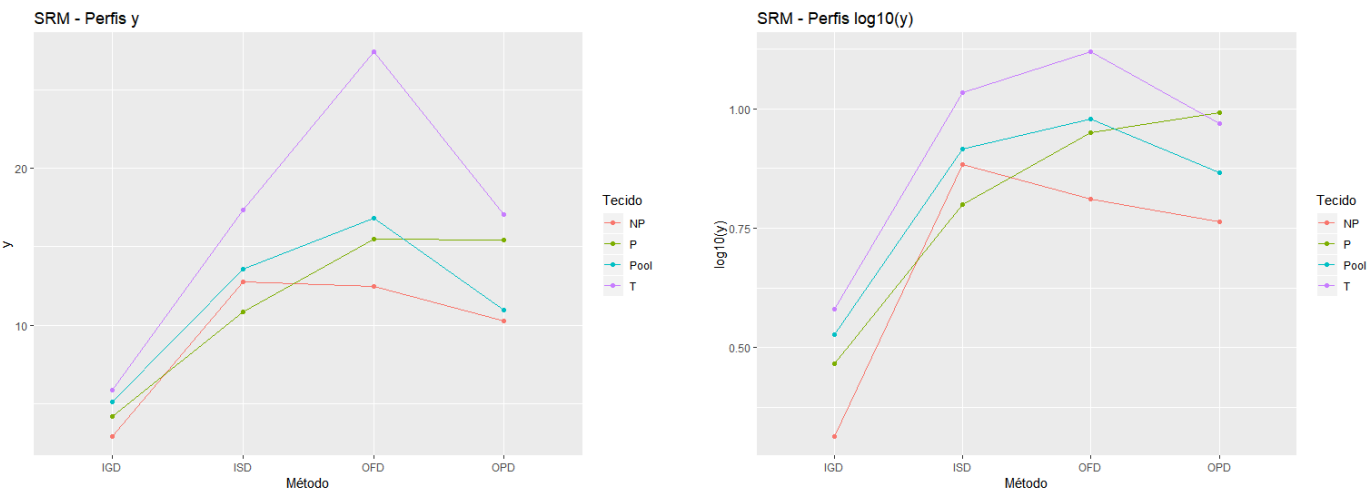


\subsubsection{Modelo de normalização}

Nesta seção serão apresentados os resultados obtidos por meio dos modelos de normalização propostos no Capítulo 4 para o experimento SRM e serão comparados os diversos métodos de preparação utilizados no experimento. A escolha do modelo que melhor se ajusta aos dados será dada pela precisão das estimativas, comportamento dos resíduos e pelo critério AIC-REML.

$\mathrm{Na}$ Tabela 5.3 estão apresentadas as principais medidas obtidas para os modelos estimados, quais sejam, as estimativas dos efeitos fixos e efeitos aleatórios preditos, as componentes de variância estimadas, assim como as medidas AIC - REML, AIC ML e $\log (L)$.

É possível perceber que, em geral, a precisão das estimativas dos efeitos fixos aumenta de acordo com a complexidade do modelo. De forma geral, observa-se que o método IGD (em gel) possuí as menores quantificações entre os demais métodos avaliados. Para os modelos M3 e M4, que consideram a presença de heterocedasticidade dos erros, é possível observar que o tecido Pool representa o tecido com menor a variabilidade e que ao se considerar o efeito do método dentro do tecido para explicar a variabilidade dos dados, o método de preparação IGD possui a menor variabilidade entre os demais métodos avaliados, sendo que esse comportamento é observado entre quase todos os tecidos, com exceção ao tecido Pool em que o método OPD apresenta uma variabilidade menor igual a 0,3552 .

Temos ainda, que a inclusão de efeitos aleatórios devido ao tecido e ao método dentro do tecido é relevante para explicar a variabilidade dos dados obtidos pelo experimento SRM. A escolha do melhor modelo se deu por meio dos critérios de seleção, quais sejam, AIC REML, AIC ML e $\log (L)$, foi considerado ainda a praticidade em identificar por meio das estimativas das componentes de variância desse modelo, o método de preparação com menor variabilidade.

Vale ressaltar que com a utilização dos resíduos dos modelos de normalização variações sistemáticas serão removidas para melhor identificar peptídeos com comportamento diferencial (análise de significância). Uma vez que o intuito nesse tipo de análise é considerar somente a variação biológica presente nas amostras, retirando qualquer tipo de variação devido à técnica utilizada no experimento (método de digestão, processo de randomização, etc.).

Além de comparar os modelos de normalização por meio da precisão das estimativas dos efeitos fixos e dos critérios de seleção de modelos, foram realizados testes estatísticos para comparar os modelos e foram gerados os gráficos dos resíduos para avaliar a qualidade do ajuste.

Foram estimados testes de Razão de Verossimilhança para comparar os modelos de normalização estimados e selecionar o com melhor ajuste. Por meio dos resultados obtidos tem-se que o modelo mais "complexo", M4, representado por um modelo linear misto com heterocedasticidade devido ao efeito do método dentro do tecido apresentou um melhor ajuste aos dados.

Na Figura 5.4 estão apresentados os resíduos condicionais com base na estimação dada pelo modelo M4 nota-se presença de alguns pontos discrepantes e uma pequena heterocedasticidade a ser considerada nas demais análises realizadas neste trabalho. Os resíduos condicionais, representados pelas observações sem os efeitos fixos e aleatórios estimados, serão utilizados para realização da análise de significância 
Tabela 5.3 - Estimativas com desvio padrão dos modelos ajustados

\begin{tabular}{|c|c|c|c|}
\hline Estimativas & Modelo M2 & Modelo M3 & Modelo M4 \\
\hline Intercepto (IGD) & $0,4714 \pm 0,1091$ & $0,4714 \pm 0,1089$ & $0,4711 \pm 0,1105$ \\
\hline ISD & $0,4369 \pm 0,1059$ & $0,4372 \pm 0,1056$ & $0,4386 \pm 0,1033$ \\
\hline OFD & $0,4933 \pm 0,1059$ & $0,4930 \pm 0,1064$ & $0,4932 \pm 0,1039$ \\
\hline OPD & $0,4263 \pm 0,1059$ & $0,4259 \pm 0,1056$ & $0,4264 \pm 0,1029$ \\
\hline$\hat{\sigma}_{1}^{2}\left(u_{1}\right)$ & 0,0080 & 0,0079 & 0,0086 \\
\hline$\hat{\sigma}_{2}^{2}\left(u_{2}\right)$ & 0,0038 & 0,0038 & 0,0036 \\
\hline$\hat{\sigma}^{2}$ & 0,1702 & - & - \\
\hline$\hat{\sigma}^{2}(\mathrm{NP})$ & - & 0,1601 & - \\
\hline$\hat{\sigma}^{2}(\mathrm{P})$ & - & 0,1837 & - \\
\hline$\hat{\sigma}^{2}(\mathrm{Pool})$ & - & 0,1517 & - \\
\hline$\hat{\sigma}^{2}(\mathrm{~T})$ & - & 0,1834 & - \\
\hline$\hat{\sigma}^{2}(\mathrm{NP}: \mathrm{IGD})$ & - & - & 0,1026 \\
\hline$\hat{\sigma}^{2}(\mathrm{NP}: \mathrm{ISD})$ & - & - & 0,1658 \\
\hline$\hat{\sigma}^{2}(\mathrm{NP}: \mathrm{OFD})$ & - & - & 0,1983 \\
\hline$\hat{\sigma}^{2}(\mathrm{NP}: \mathrm{OPD})$ & - & - & 0,1739 \\
\hline$\hat{\sigma}^{2}(\mathrm{P}: \mathrm{IGD})$ & - & - & 0,1100 \\
\hline$\hat{\sigma}^{2}(\mathrm{P}: \mathrm{ISD})$ & - & - & 0,2336 \\
\hline$\hat{\sigma}^{2}(\mathrm{P}: \mathrm{OFD})$ & - & - & 0,2237 \\
\hline$\hat{\sigma}^{2}(\mathrm{P}: \mathrm{OPD})$ & - & - & 0,1680 \\
\hline$\hat{\sigma}^{2}$ (Pool:IGD) & - & - & 0,1293 \\
\hline$\hat{\sigma}^{2}($ Pool:ISD) & - & - & 0,1660 \\
\hline$\hat{\sigma}^{2}$ (Pool:OFD) & - & - & 0,1855 \\
\hline$\hat{\sigma}^{2}$ (Pool:OPD) & - & - & 0,1262 \\
\hline$\hat{\sigma}^{2}(\mathrm{~T}: \mathrm{IGD})$ & - & - & 0,1391 \\
\hline$\hat{\sigma}^{2}(\mathrm{~T}: \mathrm{ISD})$ & - & - & 0,1607 \\
\hline$\hat{\sigma}^{2}(\mathrm{~T}: \mathrm{OFD})$ & - & - & 0,2374 \\
\hline$\hat{\sigma}^{2}(\mathrm{~T}: \mathrm{OPD})$ & - & - & 0,1961 \\
\hline $\mathrm{AIC}-\mathrm{ML}$ & 4915,04 & 4905,38 & 4810,41 \\
\hline AIC - REML & 4933,44 & 4988,04 & 4828,88 \\
\hline $\log (L)$ & $-2459,72$ & $-2451,90$ & $-2392,44$ \\
\hline
\end{tabular}


por representarem quantificações sem presença de variação técnica.

Figura 5.4 - M4: Modelo Linear Misto Heterocedástico devido ao efeito de Método dentro de Tecido
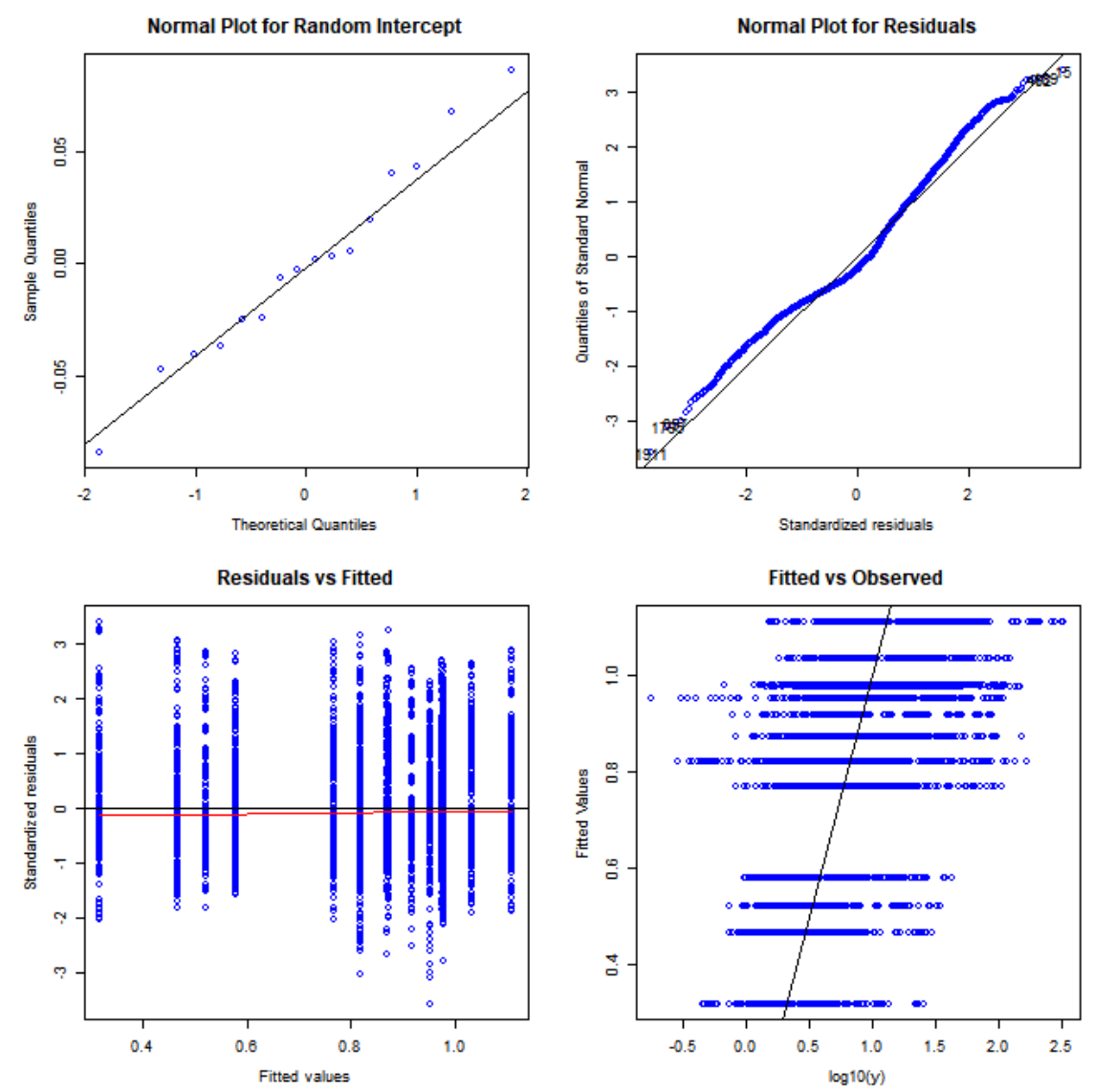

Por fim, foi comparado o comportamento das observações pré- e pós-normalização (observações originais na escala logarítmica e resíduos condicionais do Modelo M4), com o intuito de avaliar se foi possível retirar o efeito da técnica utilizada no experimento. Na Figura 5.5 têm-se as quantificações dos peptídeos para o experimento SRM pré- e pós-normalização, em que é possível perceber comportamentos diferentes entre os dois cenários. Considerando a normalização dada pelo ajuste do modelo estatístico M4, as observações se distribuem de forma semelhante (medida central), porém nota-se a presença de heterocedasticidade que deve ser considerada na realização das demais análises. 
Figura 5.5 - SRM - Quantificação de Peptídeos na escala logarítmica e normalizada

(a) SRM - Quantificação de Peptídeos na escala logarítmica

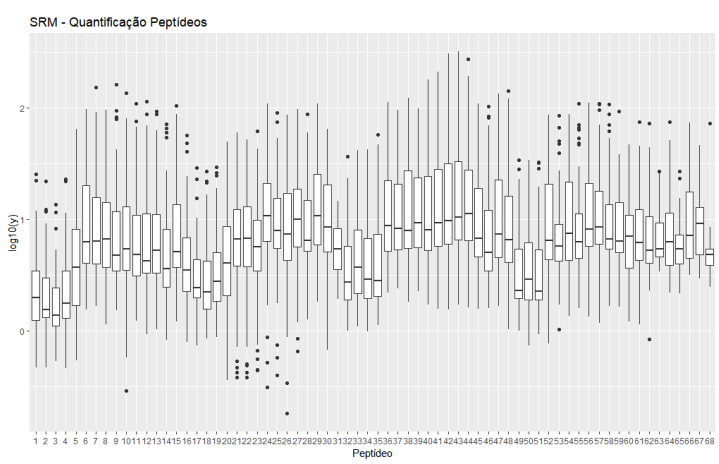

(c) SRM - Quantificação de Peptídeos considerando $\mathrm{M}(\mathrm{T})$ na escala logarítmica

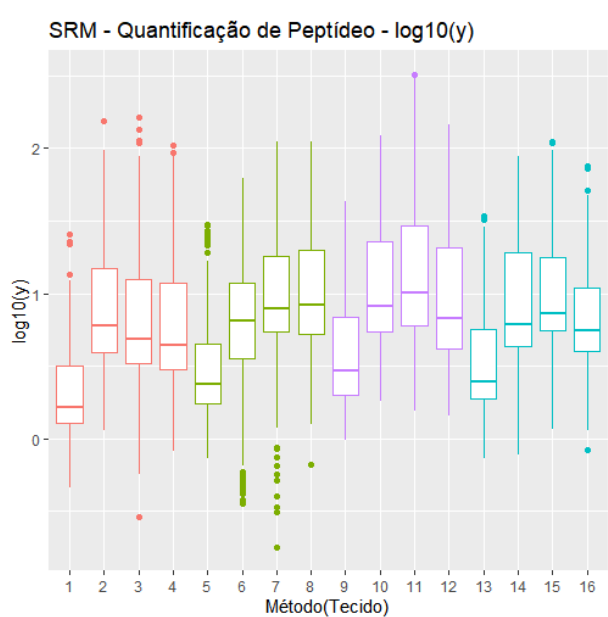

(b) SRM - Quantificação de Peptídeos após normalização

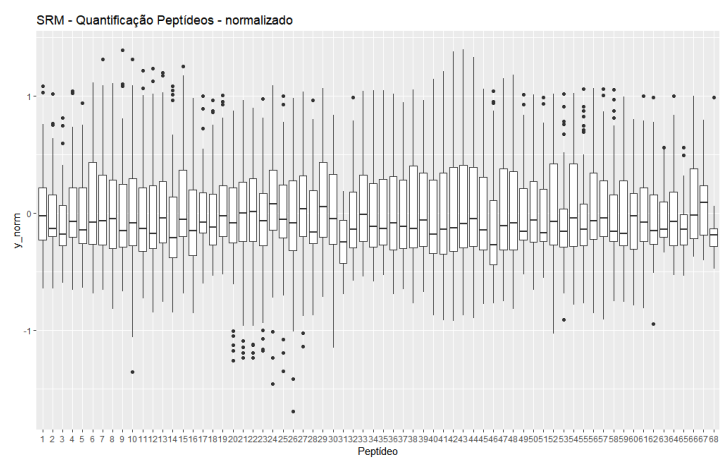

(d) SRM - Quantificação de Peptídeos considerando $\mathrm{M}(\mathrm{T})$ normalizada

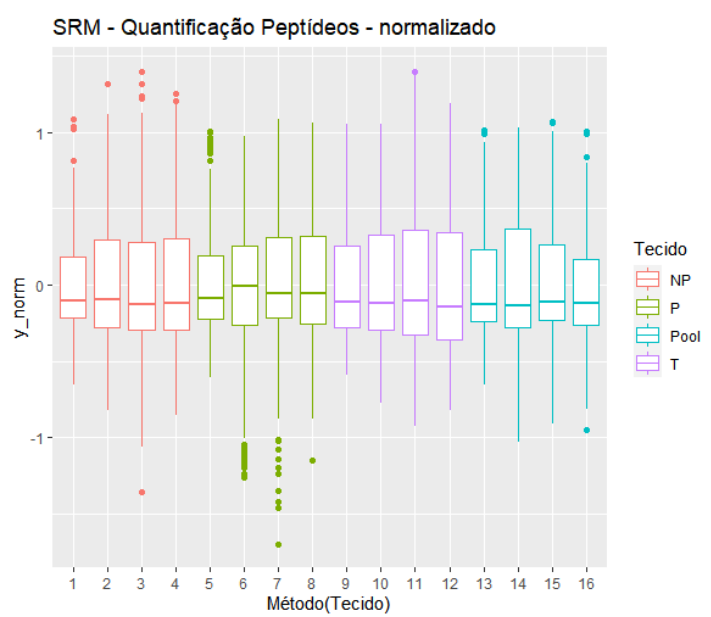




\subsubsection{Modelo de significância}

Nesta seção serão apresentados os resultados obtidos pelo modelo de significância para detecção de peptídeos com expressão diferencial entre os métodos e tecidos avaliados. Para tal, serão considerados os resíduos condicionais obtidos pelo modelo de normalização, como as variáveis respostas para realização dos testes de comparação múltipla entre métodos e tecidos. Os gráficos vulcão serão utilizados para visualização dos peptídeos com comportamento significativamente diferente.

Dessa forma, considerando o modelo de significância descrito na Seção 4.2, foram gerados os gráficos vulcão para todos os contrastes entre os pares de métodos dentro de tecido, a partir deles foram identificados os peptídeos significantes e com maiores magnitudes de mudança (fold-change $>2$ ), indicados pela cor vermelha nos gráficos.

Nota-se que para a maioria dos contrastes estimados não foi identificado nenhum peptídeo significante, com exceção para algumas comparações dentro do tecido da glândula parótica $(\mathrm{P})$. Mais precisamente, tem-se que para as seguintes comparações ISD - IGD, OFD - ISD e OPD - ISD, foi possível identificar um peptídeo significante (IPVGPETLGR).

Figura 5.6 - Gráficos vulcão para todos os contrastes entre pares de Métodos dentro de Tecido. Indicação de peptídeos significantes em vermelho ( $p$-valor $<p$-valor ajustado e |magnitude de mudança $\mid>2)$ )
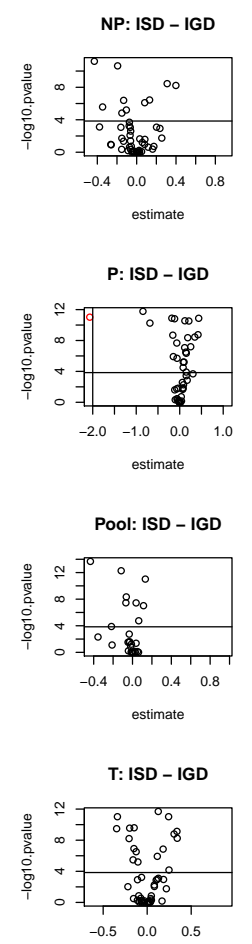

$\begin{array}{lll}-0.5 & 0.0 & 0.5\end{array}$

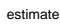

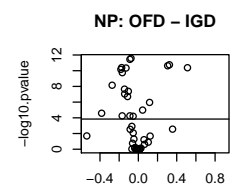
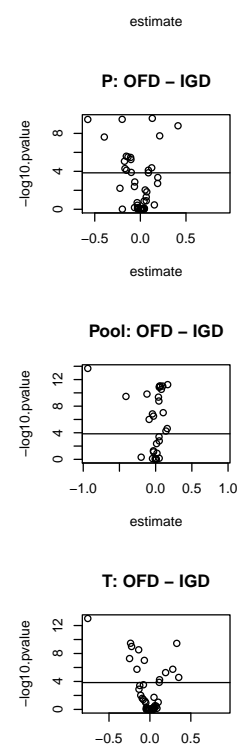

$-0.5 \quad 0.0 \quad 0.5$
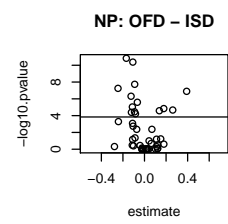

P: OFD - ISD
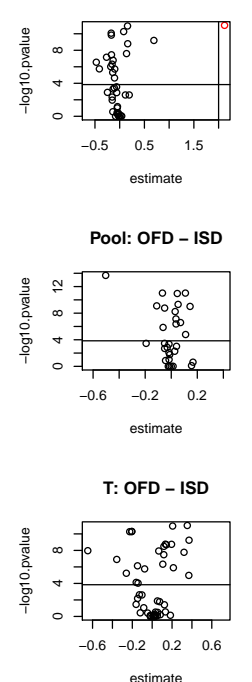
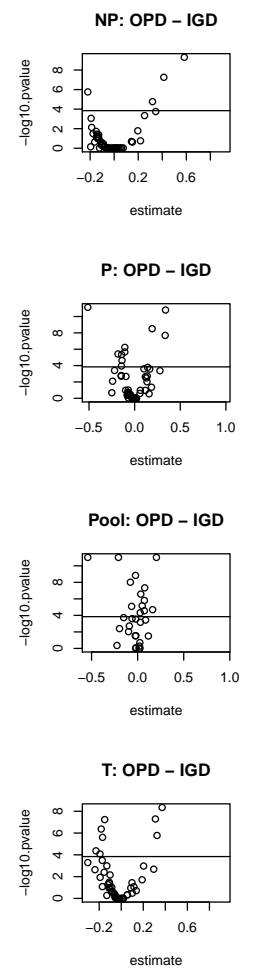
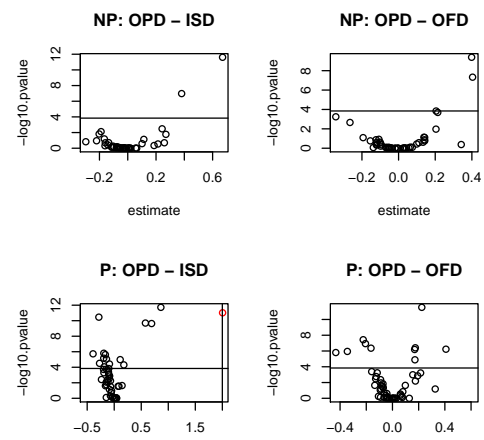

estimate
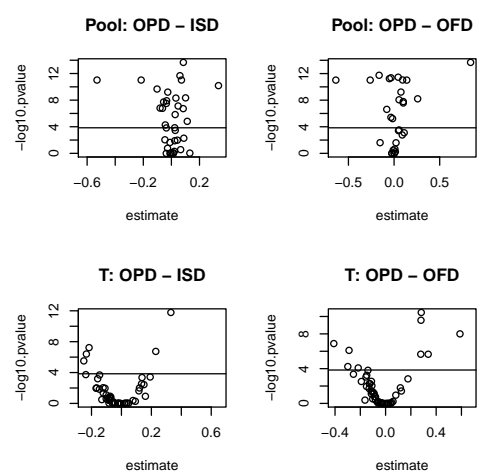


\subsection{Experimento DDA - noNorm Peptídeos}

Nesta seção serão apresentados os resultados obtidos pelos modelos de normalização e de significância para o experimento DDA noNorm Peptídeo. O experimento DDA noNorm Peptídeo compõe as quantificações de 41703 peptídeos, essas quantificações não passaram por nenhum processo de normalização, diferentemente dos experimentos TIC, que também serão avaliados para efeito de comparação.

Primeiramente serão estimados os modelos de normalização, que serão comparados quanto às estimativas dos efeitos fixos (métodos de preparação), à precisão dessas estimativas, e em relação aos critérios AIC REML e AIC ML. Após a seleção do modelo de normalização com o melhor ajuste, será estimado o modelo de significância para identificar os peptídeos com comportamento diferencial entre os efeitos do método dentro do tecido.

Vale ressaltar que o experimento DDA consiste em realizar uma varredura por peptídeos e proteínas nos tecidos analisados. Dessa forma, é comum ter como resultado bases com milhares de peptídeos e proteínas quantificadas. Entretanto, pode-se observar uma forte presença de valores faltantes, pois nem todos os peptídeos e proteínas foram identificados em todas as amostras.

Nas Figuras 5.7 e 5.8 estão apresentados os gráficos com as quantificações dos peptídeos na escala original e logarítmica, assim como os gráficos de perfis por método dentro de tecido.

Figura 5.7 - DDA noNorm - Quantificação de Peptídeos considerando Método(Tecido)
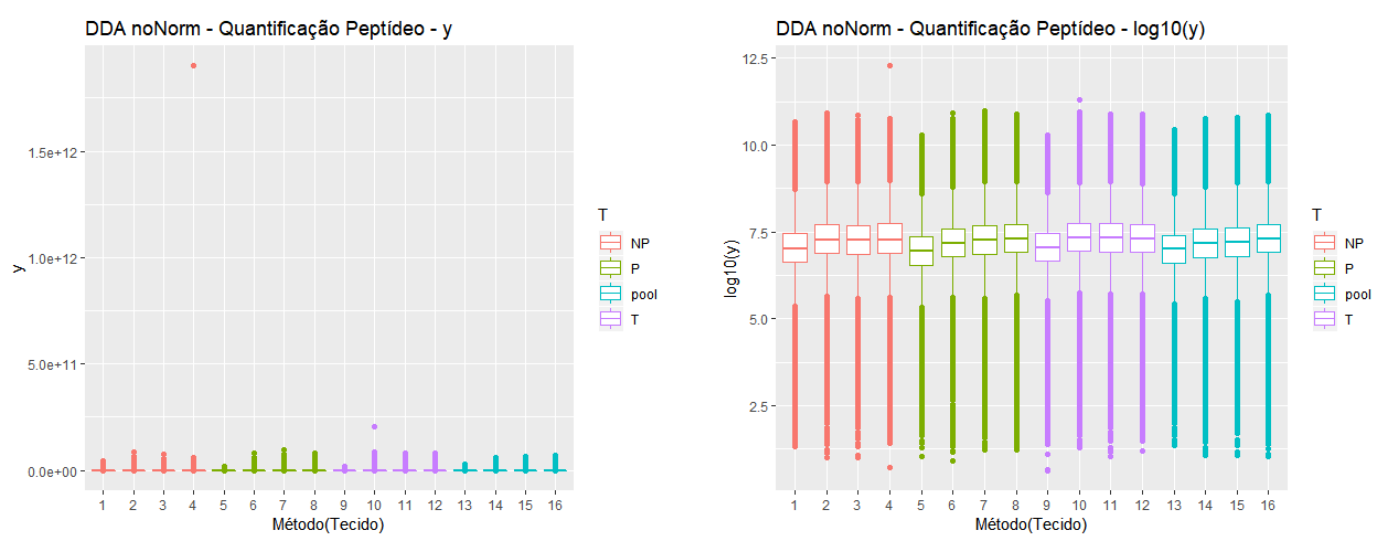

Assim como foi realizado no experimento SRM, serão ajustados primeiramente os modelos de normalização, com o intuito de retirar variações sistemáticas devido à técnica utilizada, tornando possível a identificação dos peptídeos que possuem comportamento diferente entre as condições de interesse.

\subsubsection{Modelo de normalização}

Nesta subseção serão apresentados os resultados obtidos pelos modelos M2, M3 e M4 apresentados no Capítulo 4. Esses modelos levam em consideração a estrutura dos dados por meio da inclusão de efeitos aleatórios devido ao tecido e ao método dentro de tecido. As estimativas dos modelos serão comparadas, assim como suas 
Figura 5.8 - DDA noNorm Peptídeos - Gráfico de Perfis
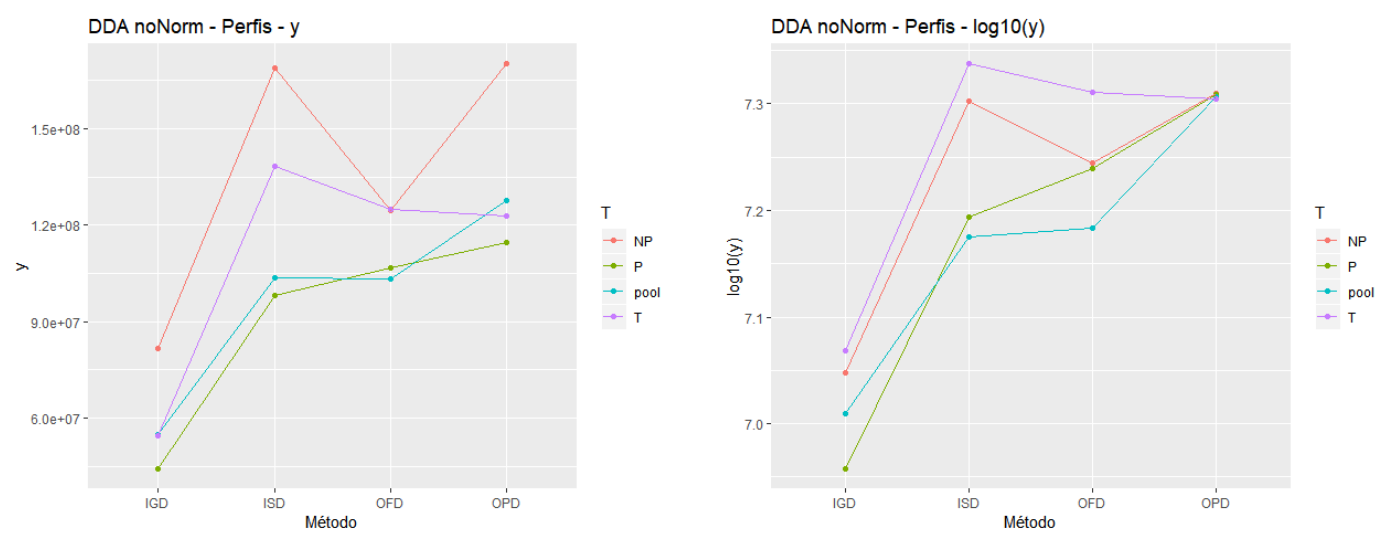

precisões. A seleção do modelo final para normalização dos dados será dada por meio da comparação das medidas AIC - REML e $\log (L)$ e por meio da realização de testes estatísticos.

Avaliando os resultados obtidos pelos modelos de normalização apresentados na Tabela 5.4, têm-se que as estimativas das componentes de variância dos modelos estimados indicam que o tecido das amídalas $(\mathrm{T})$ possui a menor variabilidade entre os demais tecidos. Além disso, têm-se que os métodos IGD e ISD possuem as menores variabilidades entre os métodos avaliados a depender do tecido estudado. Em relação à abundância de peptídeos foi possível observar que o método IGD, assim como no experimento SRM, possui as menores quantificações e, em contraste com esse resultado, o método OPD possui as maiores quantificações médias dentre os métodos de preparação avaliados.

Baseando-se nos resultados obtidos pelos testes de Razão de Máxima verossimilhanças e nas medidas de AIC e $\log (L)$, tem-se que o modelo M4 é o que melhor se ajusta aos dados, em que sua complexidade permite identificar o método de preparação com as quantificações mais precisas.

Ainda, por meio do gráfico de resíduo do modelo M4 apresentado na Figura 5.9, observa-se que devido à presença de valores discrepantes, os resíduos condicionais não se aproximam de uma distribuição normal, porém possuem um comportamento próximo de ser homocedástico.

Na Figura 5.10 foram comparadas as quantificações dos peptídeos na escala logarítmica e normalizada. Sendo possível perceber que após a normalização das quantificações os tecidos avaliados possuem comportamento semelhante quanto à media e variabilidade.

\subsubsection{Modelo de significância}

Nesta subseção serão apresentados os resultados da análise de significância por meio da utilização dos resíduos condicionais obtidos pelo modelo de normalização M4. Portanto, serão identificados os peptídeos que possuem comportamento diferencial entre os efeitos de método dentro de tecido. Para isso serão realizados testes de comparações múltiplas com ajuste no nível descritivo por FDR e serão utilizados gráficos vulcão para visualização dos resultados. 
Tabela 5.4 - Estimativas com desvio padrão dos modelos ajustados

\begin{tabular}{|c|c|c|c|}
\hline Estimativas & Modelo M2 & Modelo M3 & Modelo M4 \\
\hline Intercepto (IGD) & $7,0209 \pm 0,0523$ & $7,0209 \pm 0,0541$ & $7.0209 \pm 0,0536$ \\
\hline ISD & $0,2312 \pm 0,0619$ & $0,2312 \pm 0,0642$ & $0.2312 \pm 0,0635$ \\
\hline OFD & $0,2233 \pm 0,0619$ & $0,2233 \pm 0,0642$ & $0.2233 \pm 0,0635$ \\
\hline OPD & $0,2865 \pm 0,0619$ & $0,2865 \pm 0,0642$ & $0.2865 \pm 0,0635$ \\
\hline$\hat{\sigma}_{1}^{2}\left(u_{1}\right)$ & 0,0013 & 0,0014 & 0,0014 \\
\hline$\hat{\sigma}_{2}^{2}\left(u_{2}\right)$ & 0,0015 & 0,0016 & 0,0016 \\
\hline$\hat{\sigma}^{2}$ & 0,5166 & - & - \\
\hline$\hat{\sigma}^{2}(\mathrm{NP})$ & - & 0,5542 & - \\
\hline$\hat{\sigma}^{2}(\mathrm{P})$ & - & 0,5093 & - \\
\hline$\hat{\sigma}^{2}(\mathrm{Pool})$ & - & 0,4915 & - \\
\hline$\hat{\sigma}^{2}(\mathrm{~T})$ & - & 0,5163 & - \\
\hline$\hat{\sigma}^{2}(\mathrm{NP}: \mathrm{IGD})$ & - & - & 0,5434 \\
\hline$\hat{\sigma}^{2}(\mathrm{NP}: \mathrm{ISD})$ & - & - & 0,5274 \\
\hline$\hat{\sigma}^{2}(\mathrm{NP}: \mathrm{OFD})$ & - & - & 0,6045 \\
\hline$\hat{\sigma}^{2}(\mathrm{NP}: \mathrm{OPD})$ & - & - & 0,5419 \\
\hline$\hat{\sigma}^{2}(\mathrm{P}: \mathrm{IGD})$ & - & - & 0,4833 \\
\hline$\hat{\sigma}^{2}(\mathrm{P}: \mathrm{ISD})$ & - & - & 0,4815 \\
\hline$\hat{\sigma}^{2}(\mathrm{P}: \mathrm{OFD})$ & - & - & 0,5717 \\
\hline$\hat{\sigma}^{2}(\mathrm{P}: \mathrm{OPD})$ & - & - & 0,5037 \\
\hline$\hat{\sigma}^{2}$ (Pool:IGD) & - & - & 0,4787 \\
\hline$\hat{\sigma}^{2}($ Pool:ISD) & - & - & 0,4918 \\
\hline$\hat{\sigma}^{2}$ (Pool:OFD) & - & - & 0,5852 \\
\hline$\hat{\sigma}^{2}$ (Pool:OPD) & - & - & 0,5110 \\
\hline$\hat{\sigma}^{2}(\mathrm{~T}: \mathrm{IGD})$ & - & - & 0,4576 \\
\hline$\hat{\sigma}^{2}(\mathrm{~T}: \mathrm{ISD})$ & - & - & 0,4779 \\
\hline$\hat{\sigma}^{2}(\mathrm{~T}: \mathrm{OFD})$ & - & - & 0,5506 \\
\hline$\hat{\sigma}^{2}(\mathrm{~T}: \mathrm{OPD})$ & - & - & 0,4858 \\
\hline $\mathrm{AIC}-\mathrm{ML}$ & 3733147 & 3731624 & 3727615 \\
\hline AIC - REML & 3733170 & 3731648 & 3727638 \\
\hline $\log (L)$ & -1866578 & -1865814 & -1863797 \\
\hline
\end{tabular}


Figura 5.9 - M4: Modelo Linear Misto Heterocedástico devido ao efeito de Método dentro de Tecido
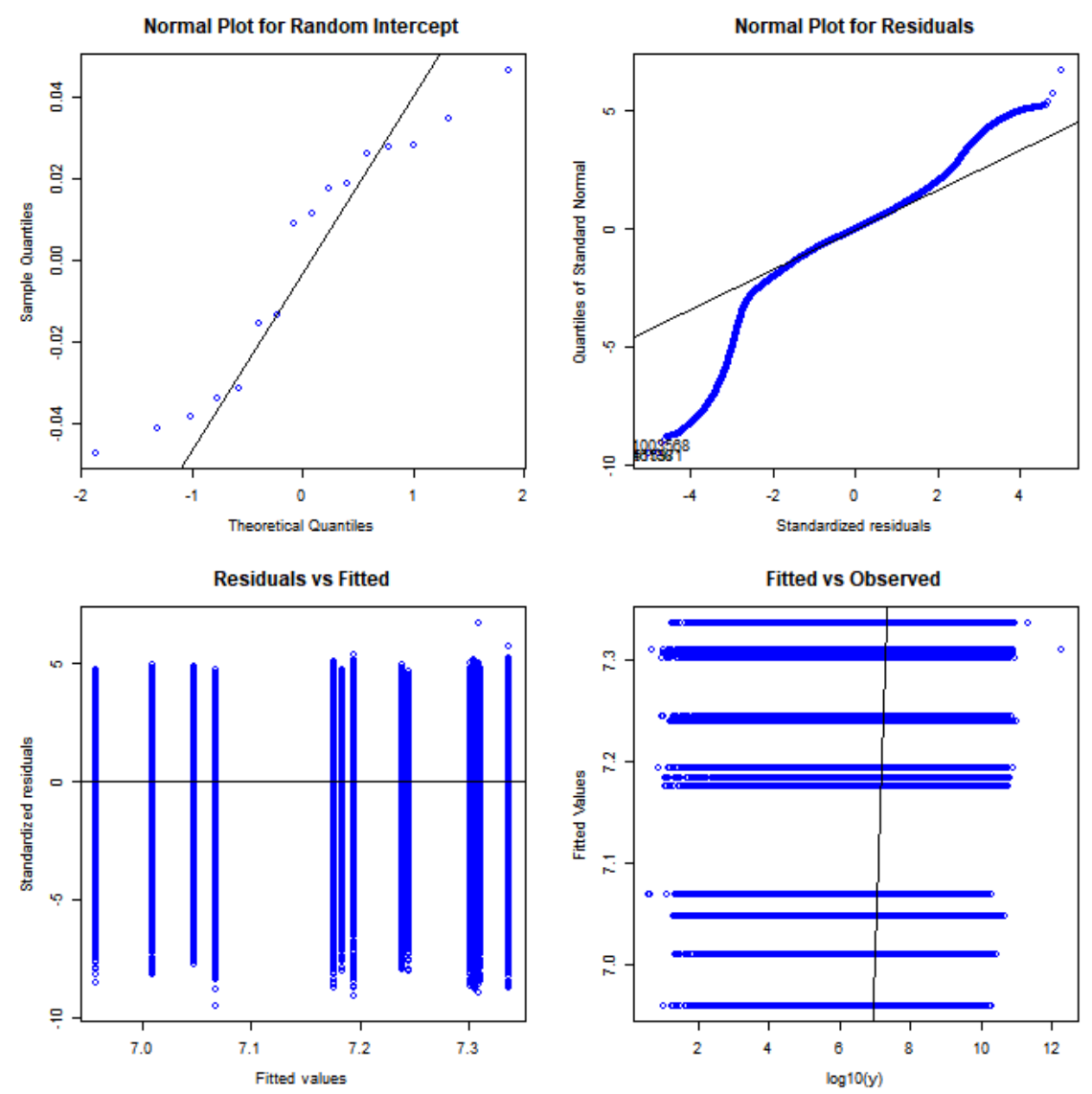

Figura 5.10 - DDA noNorm - Quantificação de Peptídeos na escala logarítmica e normalizada

(a) DDA noNorm - Quantificação de

Peptídeos considerando $\mathrm{M}(\mathrm{T})$ na escala logarítmica

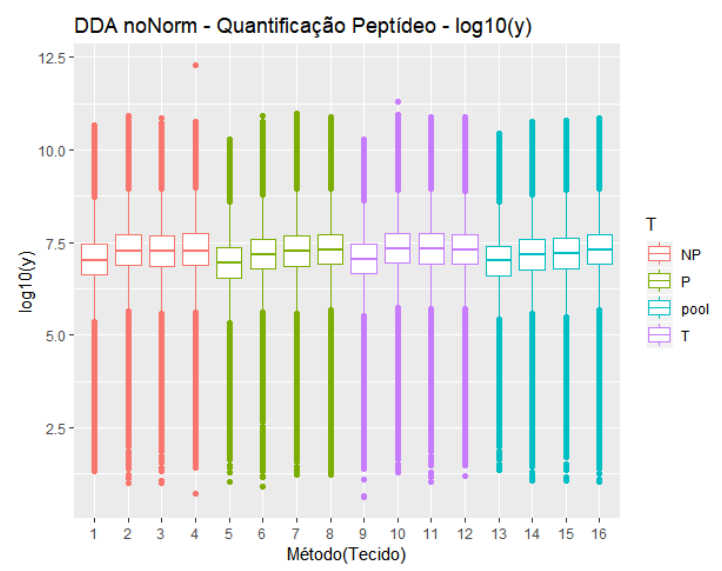

(b) DDA noNorm - Quantificação de Peptídeos considerando $\mathrm{M}(\mathrm{T})$ normalizada

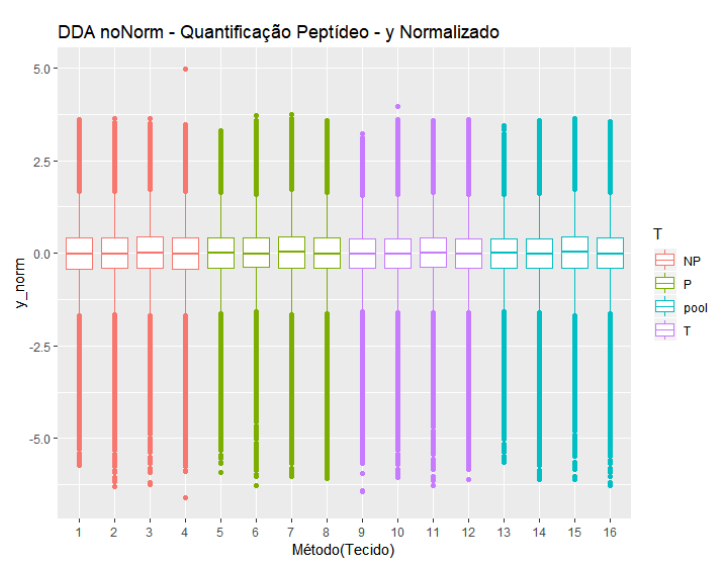


Primeiramente, o modelo de significância apresentado no Capítulo 4 foi ajustado para cada peptídeo quantificado no experimento. Vale mencionar que as amostras com valores faltantes, ou seja, em que não foi possível quantificar todos os peptídeos, foram retiradas para estimação do modelo de significância, resultando no recorte de 12612 do total de 41703 peptídeos quantificados. Na Figura 5.11 estão apresentados os gráficos de vulcão gerados para o experimento.

Figura 5.11 - Gráficos vulcão para todos os contrastes entre pares de Métodos dentro de Tecido. Indicação de peptídeos significantes em vermelho ( $p$-valor $<$ $p$-valor ajustado e $\mid$ magnitude de mudança $\mid>2)$ )
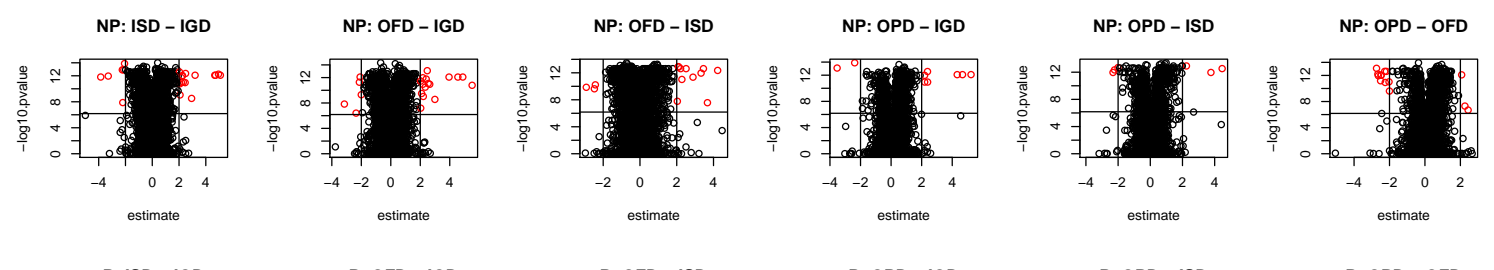

P: ISD - IGD

P: OFD - IGD
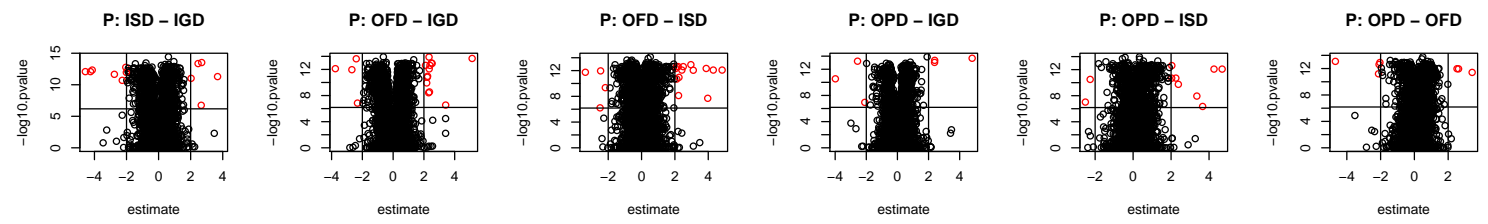

pool: ISD - IGD
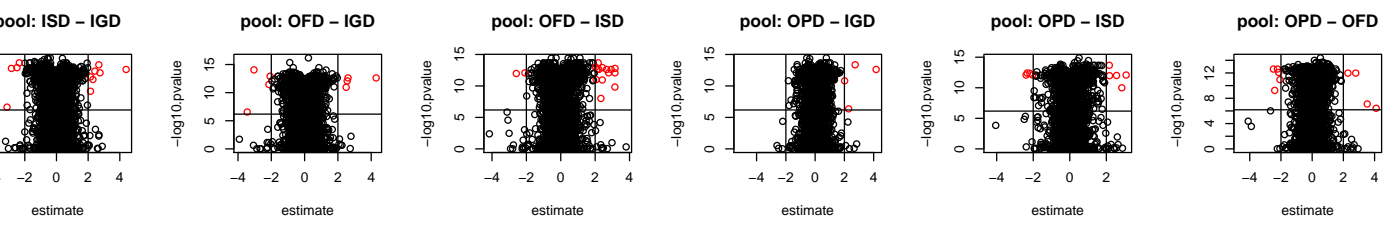

T: ISD - IGD
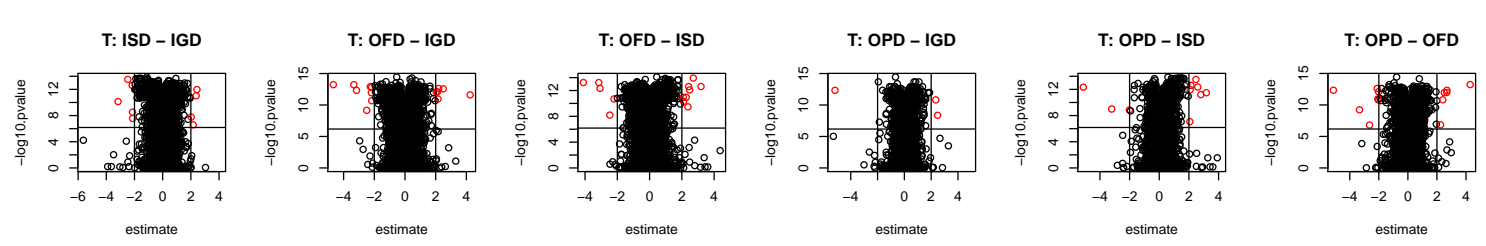

Com base nos resultados apresentados, foi possível identificar 164 peptídeos considerados significantes entre os efeitos de método dentro de tecido. Tem-se como destaque o contraste NP: ISD - IGD em que foram identificados 37 dos 164 peptídeos com comportamento diferencial no experimento.

Os peptídeos QGFFPDSVNK e QSLQVIESAMER foram considerados significantes em quase todos os contrastes avaliados, com exceção dos contrastes que comparam os métodos ISD e IGD para todos os tecidos. Além disso, têm-se que 48 dos 164 peptídeos foram significantes somente em um contraste, 46 em dois e 53 em três.

Os resultados obtidos pelo modelo de significância para o experimento DDA noNorm Peptídeos serão comparados na próxima seção com os resultados do experimento DDA - TIC Peptídeos, com o intuito de se avaliar possíveis diferenças devido ao método de normalização utilizado. 


\subsection{Experimento DDA - TIC Peptídeos}

Uma prática comum na Bioinformática consiste em realizar a normalização dos dados obtidos pelos experimentos SRM e DDA por meio da técnica TIC, porém, conforme mencionado anteriormente, essa técnica possui fragilidades, como por exemplo, não se sabe ao certo quais fatores estão sendo retirados dos dados ao se realizar a normalização.

Assim, nesta seção serão comparados os resultados do modelo de significância, considerando a normalização TIC e a normalização proposta neste trabalho, com o intuito de se avaliar a semelhança com os resultados apresentados na subseção 5.2.

Por meio dos gráficos apresentados na Figura 5.12 tem-se que na média o método IGD possui as maiores quantificações dentro dos tecidos, com exceção do tecido dos pólipos nasais (NP). Esse resultado contradiz com o que vem sendo observado nos experimentos SRM e DDA noNorm Peptídeos.

Baseando-se nos dados normalizados pela técnica TIC foi ajustado o modelo de significância com o intuito de identificar peptídeos com comportamento diferencial entre os métodos dentro dos tecidos e comparar os resultados com os que foram obtidos na seção anterior. Na Figura 5.14 estão apresentados os gráficos vulcão para todos os contrastes entre pares do efeito de método dentro de tecido. Sendo que, os peptídeos indicados pela cor vermelha representam peptídeos significantes e com maiores magnitudes de mudança.

Figura 5.12 - DDA TIC Peptídeos - Quantificação de Peptídeos considerando Método(Tecido)
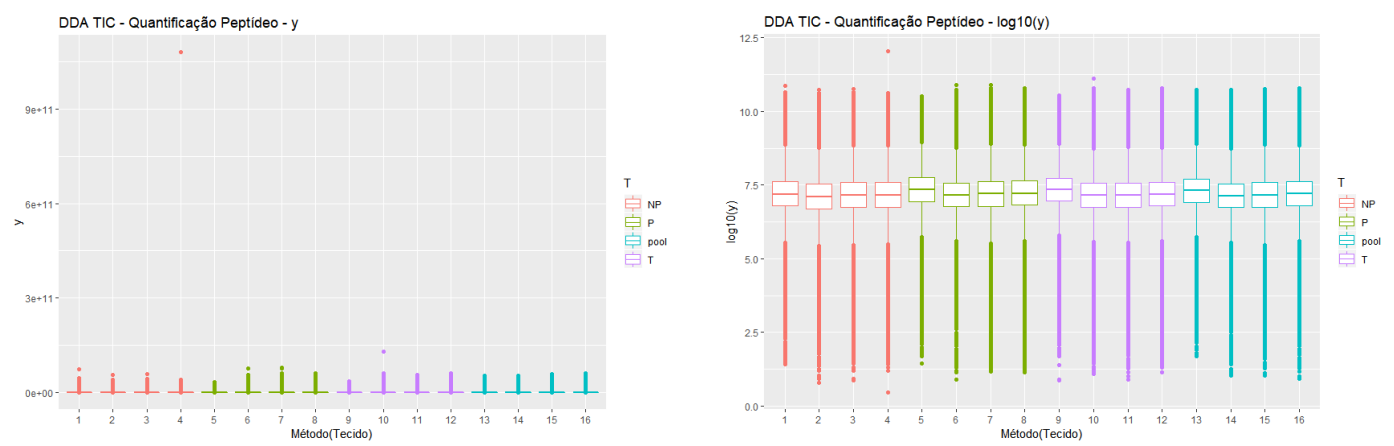

Figura 5.13 - DDA TIC Peptídeos - Gráfico de Perfis
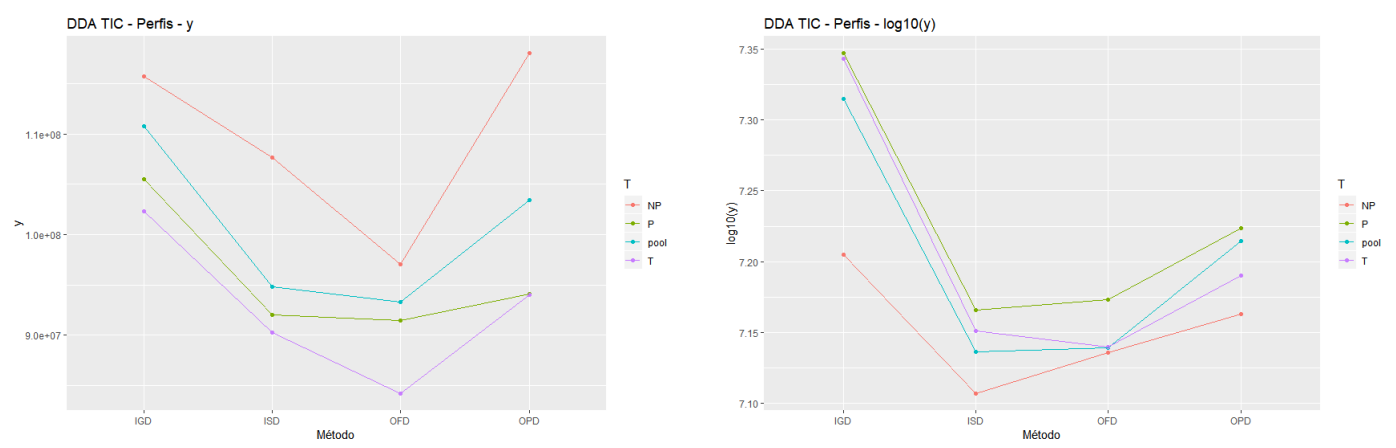


\subsubsection{Modelo de significância}

Foram identificados 188 peptídeos significantes. Assim como foi observado para o experimento DDA noNorm o peptídeo QSLQVIESAMER foi considerado significante em quase todos os contrastes avaliados com exceção dos contrastes que comparam os métodos ISD e IGD. Já para o peptídeos QGFFPDSVNK, além da não significância nos mesmos contrastes que o peptídeo QSLQVIESAMER, também não foi significante no contraste P: OPD - OFD. No demais, 66 peptídeos foram considerados significantes somente em um contraste, 47 em dois e 53 em três.

Assim como foi observado na seção anterior, tem-se que no contraste NP: ISD - IGD foram identificados o maior número de peptídeos significantes. No geral, os contrastes que comparam os métodos OPD e IGD apresentaram o menor número de peptídeos com comportamento diferencial.

Figura 5.14 - Gráficos vulcão para todos os contrastes entre pares de Métodos dentro de Tecido. Indicação de peptídeos significantes em vermelho ( $p$-valor $<$ $p$-valor ajustado e $\mid$ magnitude de mudança $\mid>2)$ )
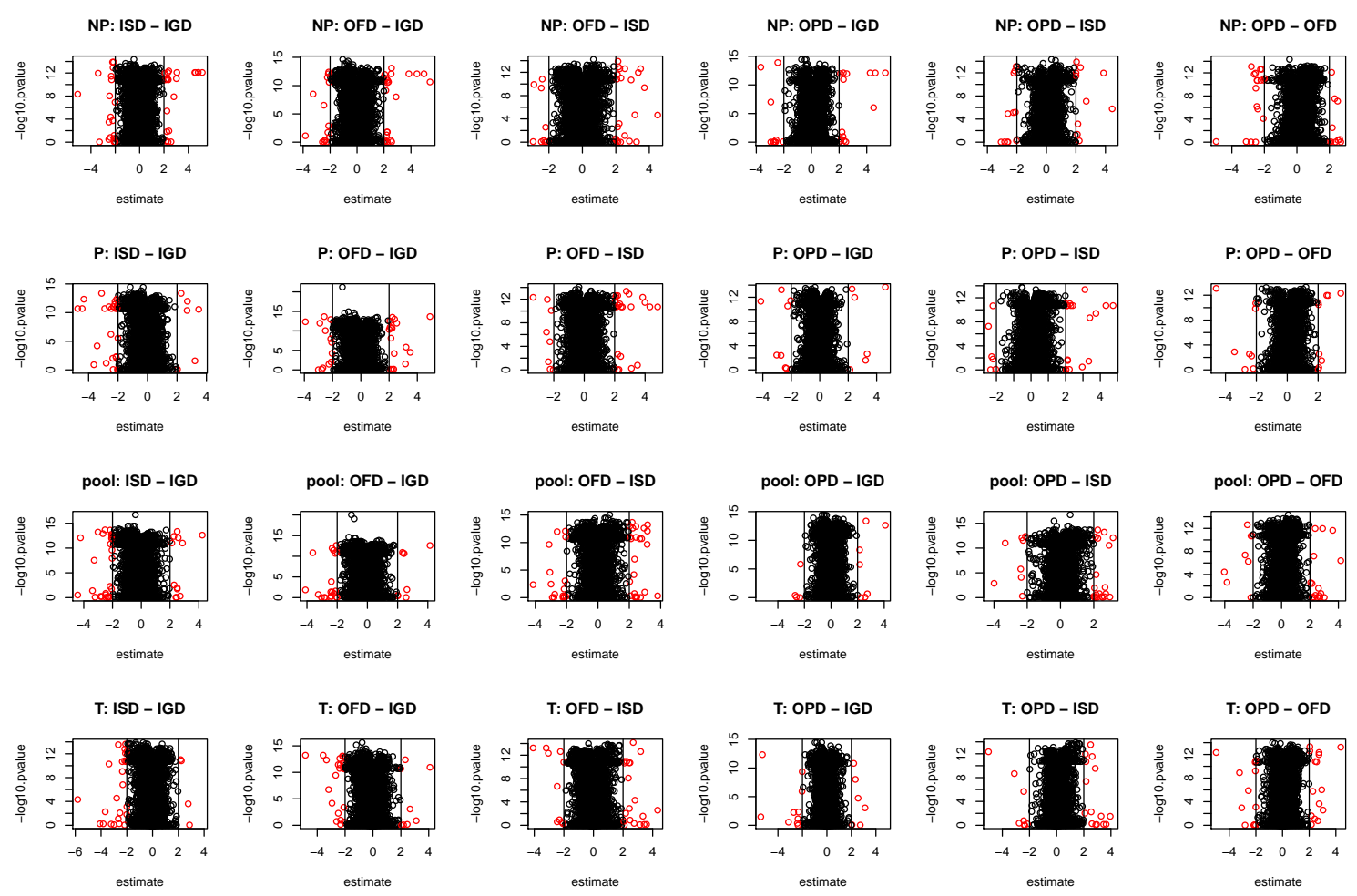

Vale ressaltar que o grupo de peptídeos identificados como significantes não é o mesmo para os experimentos DDA noNorm e DDA TIC. Sendo que, 153 dos 164 peptídeos identificados no experimento DDA noNorm também fazem parte do grupo de peptídeos identificados como significantes no experimento DDA TIC, destacando assim que o modelo de normalização escolhido influencia nos resultados obtidos. 
Tabela 5.5 - Peptídeos considerados significantes somente no experimento DDA TIC

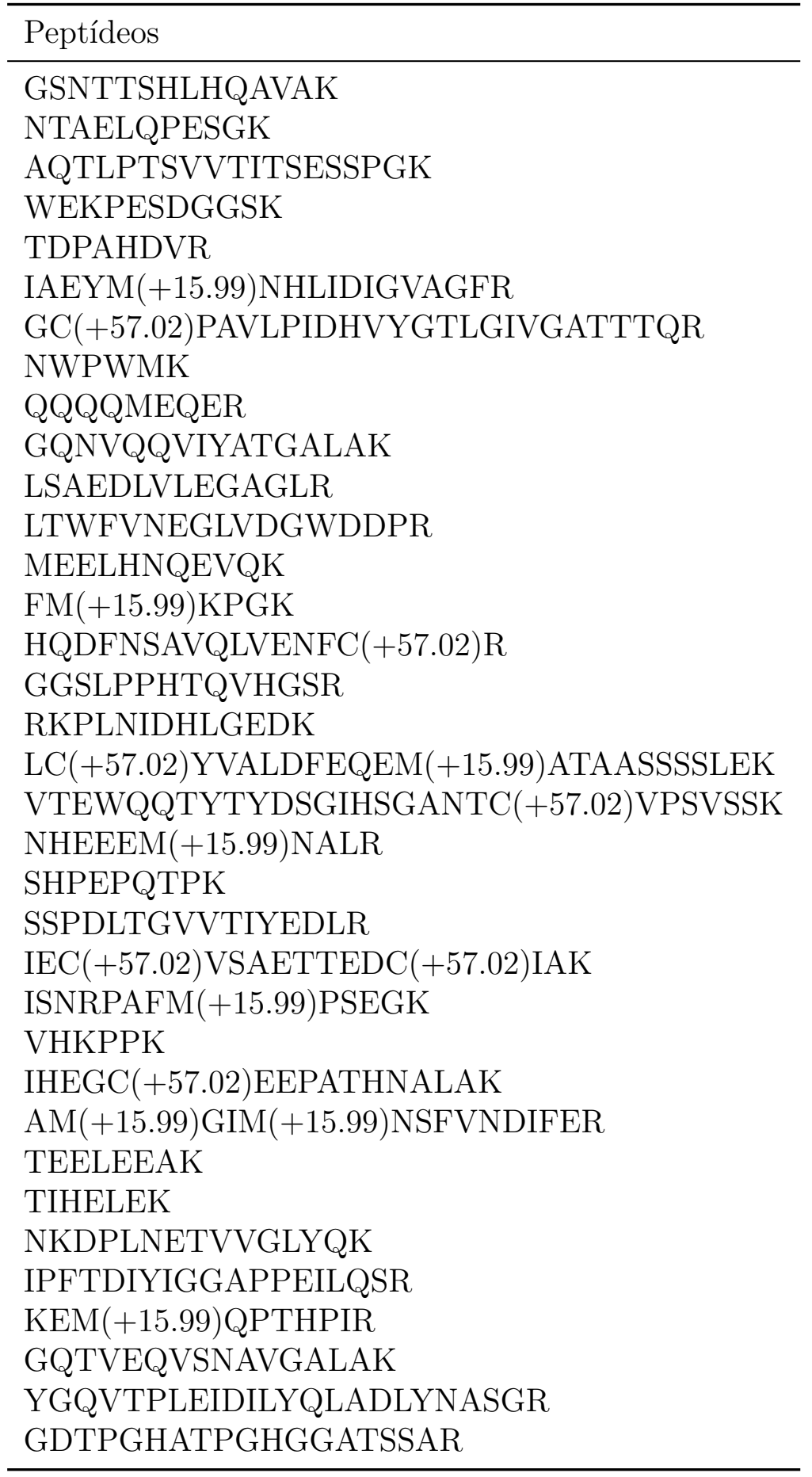




\subsection{Experimento DDA - noNorm Proteínas}

O último experimento que será avaliado neste trabalho corresponde ao experimento DDA noNorm e TIC para proteínas. Nesta seção serão apresentados os resultados do experimento noNorm, em que os dados obtidos não passaram por nenhum processo de normalização. Dessa forma, serão estimados primeiramente os modelos de normalização propostos neste trabalho e os resultados obtidos por eles serão comparados para que seja possível escolher aquele que melhor se ajusta aos dados. Por meio dos resíduos condicionais do modelo escolhido será estimado o modelo de significância, com o intuito de identificar proteínas com comportamento diferencial entre os efeitos de método dentro de tecido.

Figura 5.15 - DDA noNorm - Quantificação de Proteínas considerando Método(Tecido)
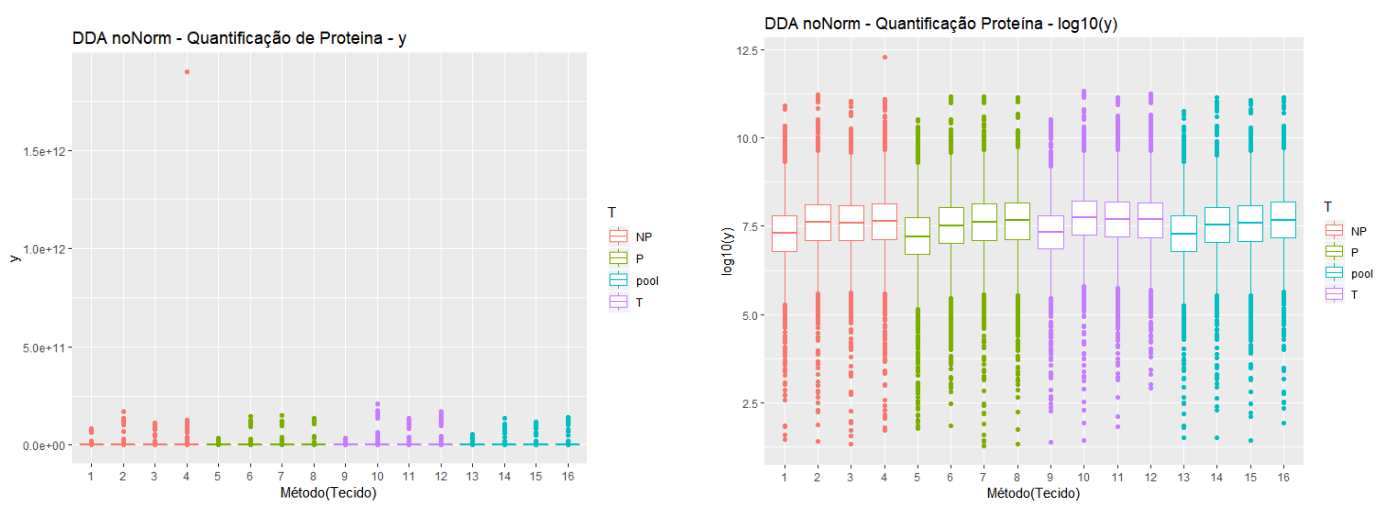

Figura 5.16 - DDA noNorm Proteína - Gráfico de Perfis
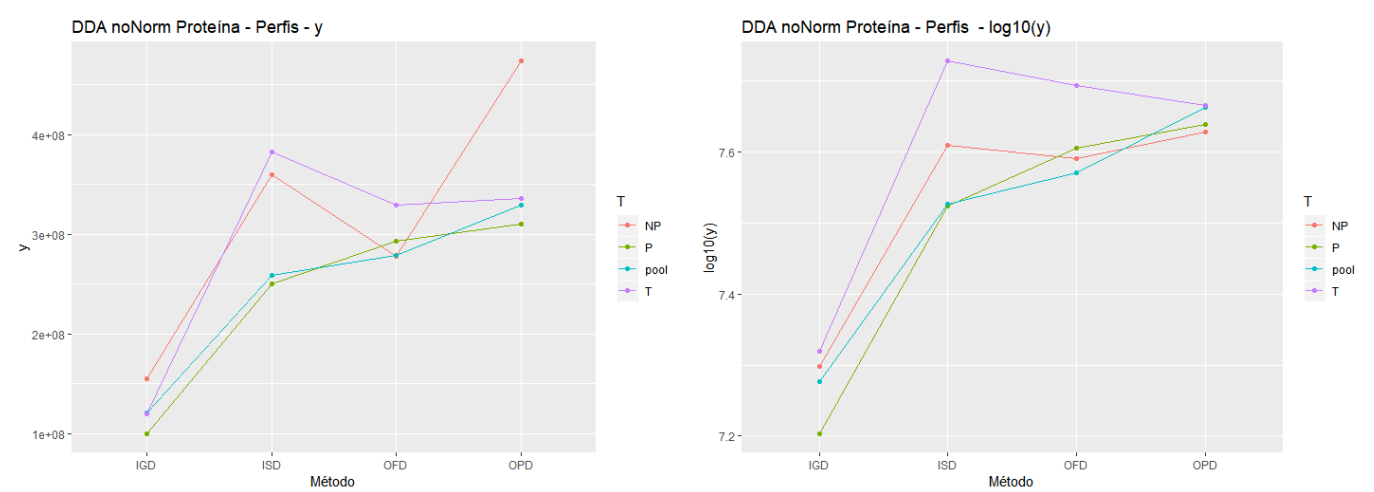

\subsubsection{Modelo de normalização}

Na Tabela 5.6 estão apresentadas as estimativas dos modelos M2, M3 e M4. Sendo possível perceber que os efeitos aleatórios são relevantes para a análise do experimento e devem ser considerados na normalização dos dados. Além disso, tem-se pelos resultados do modelo M4 que o tecido T possui a menor variabilidade entre os demais tecidos avaliados e o mesmo é observado para o método de preparação ISD. 
Nota-se ainda que o método IGD possui as menores quantificações médias, assim como foi observado nos experimentos SRM e DDA noNorm. No entanto, para o experimento DDA TIC foi observado que as quantificação para esse método foram as maiores.

Comparando os modelos por meio dos teste de razão de máxima verossimilhanças e pelos valores dos critérios AIC e $\log (L)$, tem-se que o modelo M4 apresentou o melhor ajuste por meio da consideração da heterocedasticidade dos dados dada pelo efeito de método dentro do tecido e permite comparar os métodos de preparação avaliados em relação a variabilidade de suas quantificações.

Tabela 5.6 - Estimativas com desvio padrão dos modelos estimados

\begin{tabular}{|c|c|c|c|}
\hline Estimativas & Modelo M2 & Modelo M3 & Modelo M4 \\
\hline Intercepto (IGD) & $7,2744 \pm 0,0605$ & $7.2744 \pm 0,0630$ & $7,2744 \pm 0,0622$ \\
\hline ISD & $0,3225 \pm 0,0709$ & $0.3225 \pm 0,0686$ & $0,3225 \pm 0,0729$ \\
\hline OFD & $0,3407 \pm 0,0709$ & $0.3407 \pm 0,0686$ & $0,3407 \pm 0,0729$ \\
\hline OPD & $0,3743 \pm 0,0709$ & $0.3743 \pm 0,0686$ & $0,3743 \pm 0,0729$ \\
\hline$\hat{\sigma}_{1}^{2}\left(u_{1}\right)$ & 0,0018 & 0,0023 & 0,0020 \\
\hline$\hat{\sigma}_{2}^{2}\left(u_{2}\right)$ & 0,0019 & 0,0018 & 0,0021 \\
\hline$\hat{\sigma}^{2}$ & 0,6281 & - & - \\
\hline$\hat{\sigma}^{2}(\mathrm{NP})$ & - & 0,6573 & - \\
\hline$\hat{\sigma}^{2}(\mathrm{P})$ & - & 0,6418 & - \\
\hline$\hat{\sigma}^{2}(\mathrm{Pool})$ & - & 0,6358 & - \\
\hline$\hat{\sigma}^{2}(\mathrm{~T})$ & - & 0,5809 & - \\
\hline$\hat{\sigma}^{2}(\mathrm{NP}: \mathrm{IGD})$ & - & - & 0,6673 \\
\hline$\hat{\sigma}^{2}(\mathrm{NP}: \mathrm{ISD})$ & - & - & 0,6428 \\
\hline$\hat{\sigma}^{2}(\mathrm{NP}: \mathrm{OFD})$ & - & - & 0,6515 \\
\hline$\hat{\sigma}^{2}(\mathrm{NP}: \mathrm{OPD})$ & - & - & 0,6684 \\
\hline$\hat{\sigma}^{2}(\mathrm{P}: \mathrm{IGD})$ & - & - & 0,6500 \\
\hline$\hat{\sigma}^{2}(\mathrm{P}: \mathrm{ISD})$ & - & - & 0,6068 \\
\hline$\hat{\sigma}^{2}(\mathrm{P}: \mathrm{OFD})$ & - & - & 0,6652 \\
\hline$\hat{\sigma}^{2}(\mathrm{P}: \mathrm{OPD})$ & - & - & 0,6490 \\
\hline$\hat{\sigma}^{2}($ Pool:IGD $)$ & - & - & 0,6259 \\
\hline$\hat{\sigma}^{2}($ Pool:ISD $)$ & - & - & 0,6118 \\
\hline$\hat{\sigma}^{2}(\mathrm{Pool}: \mathrm{OFD})$ & - & - & 0,6762 \\
\hline$\hat{\sigma}^{2}$ (Pool:OPD) & - & - & 0,6314 \\
\hline$\hat{\sigma}^{2}(\mathrm{~T}: \mathrm{IGD})$ & - & - & 0,5645 \\
\hline$\hat{\sigma}^{2}(\mathrm{~T}: \mathrm{ISD})$ & - & - & 0,5581 \\
\hline$\hat{\sigma}^{2}(\mathrm{~T}: \mathrm{OFD})$ & - & - & 0,5945 \\
\hline$\hat{\sigma}^{2}(\mathrm{~T}: \mathrm{OPD})$ & - & - & 0,6012 \\
\hline $\mathrm{AIC}-\mathrm{ML}$ & 533237 & 532995 & 532924 \\
\hline AIC - REML & 533259 & 533017 & 532946 \\
\hline $\log (L)$ & -266623 & -266499 & -266451 \\
\hline
\end{tabular}

Avaliando a qualidade do modelo M4 ajustado, tem-se pela Figura 5.17 que os resí- 
duos apresentam observações discrepantes, impactando na normalidade dos resíduos. No entanto, pode-se notar resíduos com comportamento próximo da homocedasticidade.

Portanto, para avaliação da significância do experimento serão utilizados os resíduos condicionais do modelo escolhido e os resultados obtidos serão apresentados na próxima subseção.

Figura 5.17 - M4: Modelo Linear Misto Heterocedástico devido ao efeito do Método dentro do Tecido
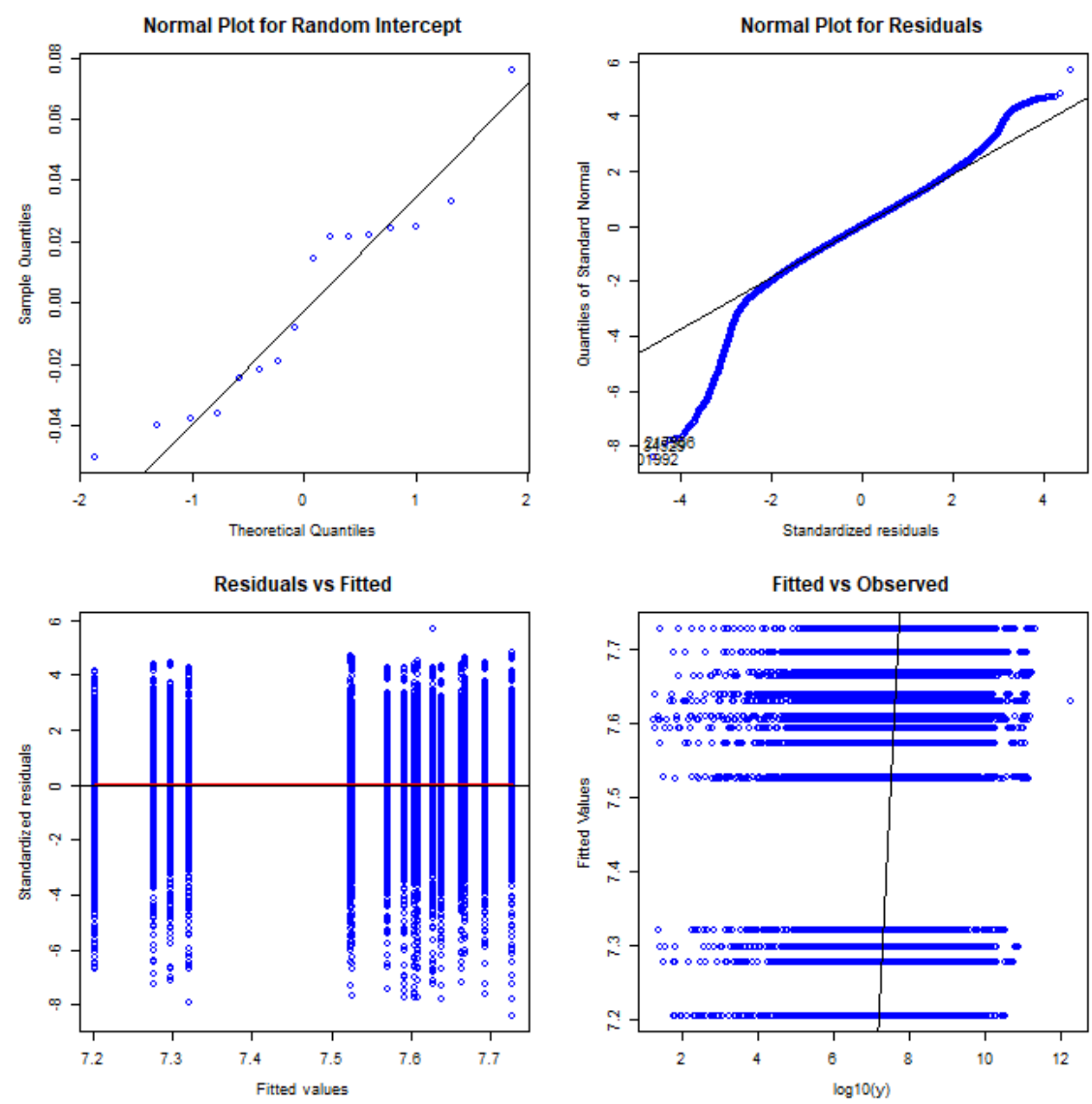

\subsubsection{Modelo de significância}

Na Figura 5.18 estão sendo comparadas as quantificações de proteínas considerando os dados pré- e pós-normalização dos dados. Nota-se que em média o comportamento das quantificações considerando o efeito de método dentro de tecido é semelhante, porém com um pequeno desvio quando à variabilidade.

Portanto, para identificação das proteínas significantes neste experimento, foi estimado o modelo de significância proposto neste trabalho e por meio dos resultados obtidos foram gerados os gráficos vulcão apresentados na Figura 5.19.

Considerando os resultados obtidos pelos gráficos, têm-se que 53 das 2289 proteínas quantificadas foram identificadas como significantes. A proteína identificada pelo 
número 3126 foi considerada significante para quase todos os contrastes com exceção dos contrastes que comparam os métodos ISD e IGD entre os tecidos avaliados.

Figura 5.18 - DDA noNorm - Quantificação de Proteínas na escala logarítmica e normalizada

(a) DDA noNorm - Quantificação de

Proteínas considerando $\mathrm{M}(\mathrm{T})$ na escala logarítmica

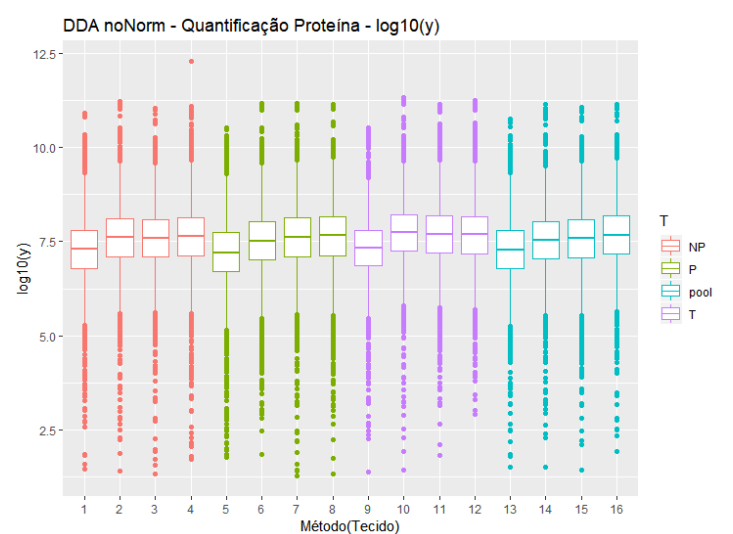

(b) DDA noNorm - Quantificação de Proteínas considerando $\mathrm{M}(\mathrm{T})$ normalizada

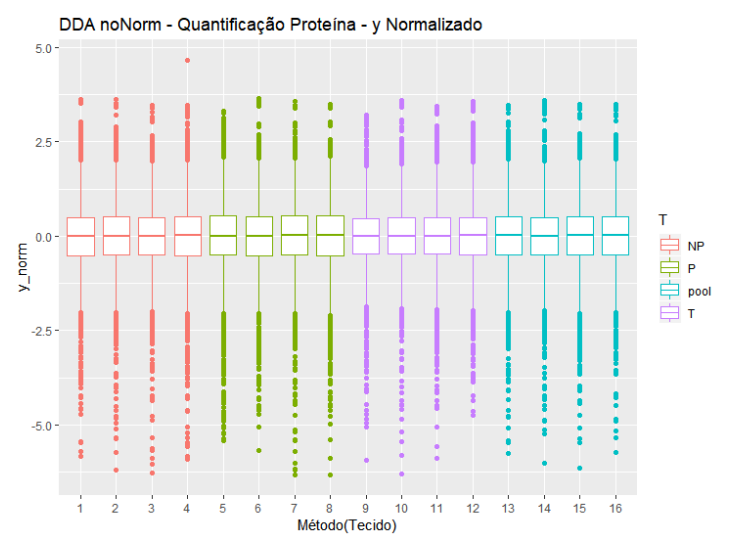


Figura 5.19 - Gráficos vulcão para todos os contrastes entre pares de Métodos dentro de Tecido. Indicação de peptídeos significantes em vermelho ( $p$-valor $<$ $p$-valor ajustado e $\mid$ magnitude de mudança $\mid>2)$ )
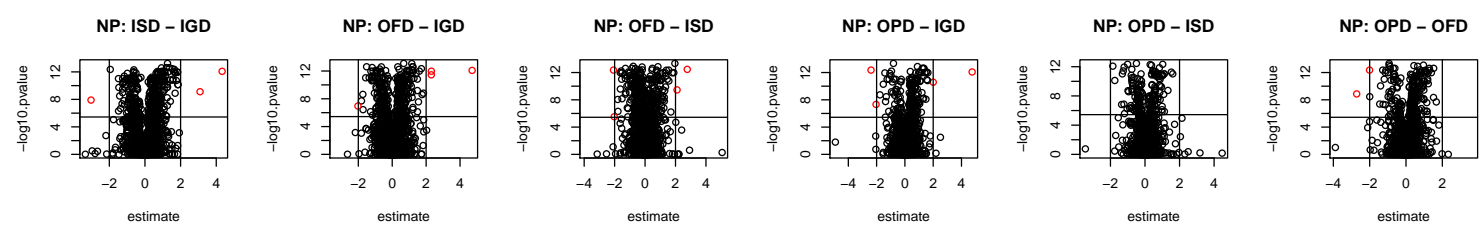

P: ISD - IGD

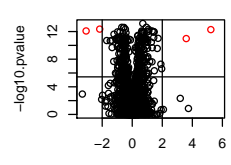

P: OFD - IGD
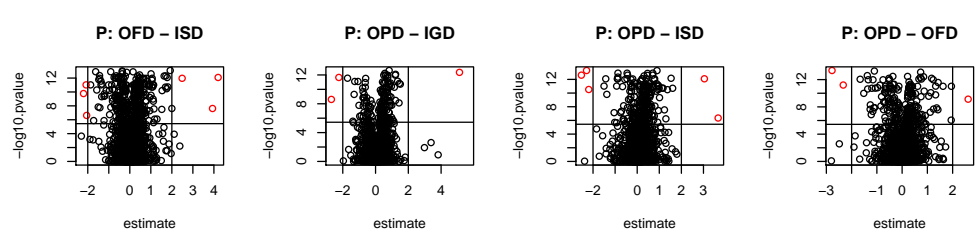

pool: ISD - IGD
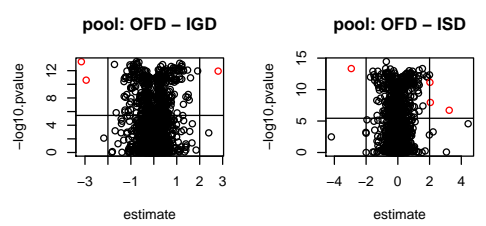

pool: OPD - IGD
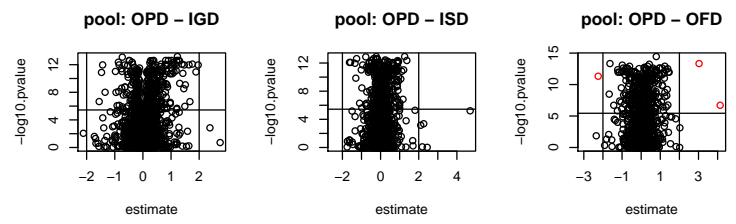

T: ISD - IGD

T: OFD - IGD

T: OFD - ISD

T: OPD - IGD

T: OPD - ISD

T: OPD - OFD
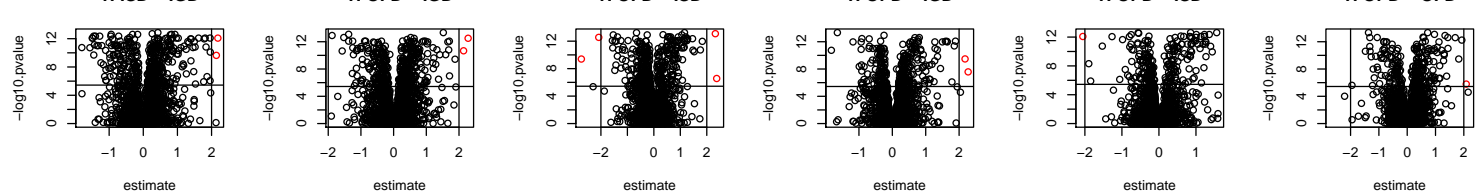


\subsection{Experimento DDA - TIC Proteínas}

Para efeito de comparação, nesta seção serão apresentados os resultados obtidos pela análise de normalização para o experimento DDA TIC Proteínas considerando os dados normalizados pela técnica TIC utilizada na Bioinformática. Portanto, será ajustado somente o modelo de significância e serão obtidos os gráficos vulcões para identificação das proteínas significantes.

Nas Figuras 5.20 e 5.21 estão comparadas as quantificações das proteínas pré- e pós-normalização dos dados pela técnica TIC. De modo geral, o método IGD possui em média as maiores quantificações de proteínas, assim como foi observado no experimento DDA peptídeos com a técnica TIC, porém oposto ao que foi encontrado nos experimentos DDA noNorm peptídeos e proteínas e no experimento SRM.

Figura 5.20 - DDA TIC - Quantificação de Proteínas considerando Método(Tecido)
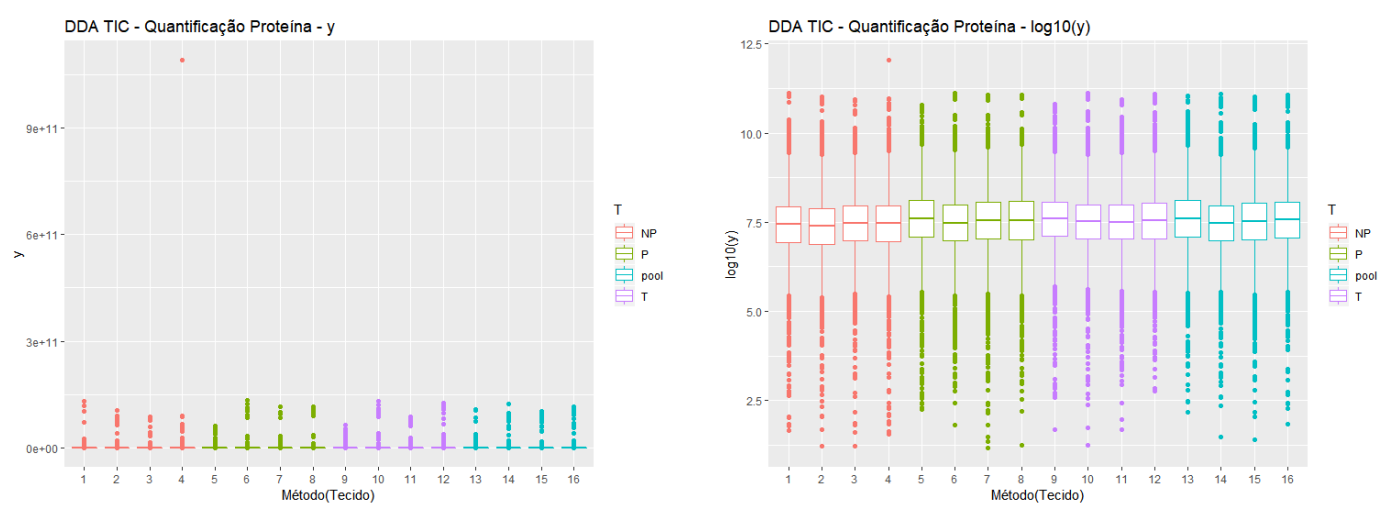

Figura 5.21 - DDA TIC Proteína - Gráfico de Perfis
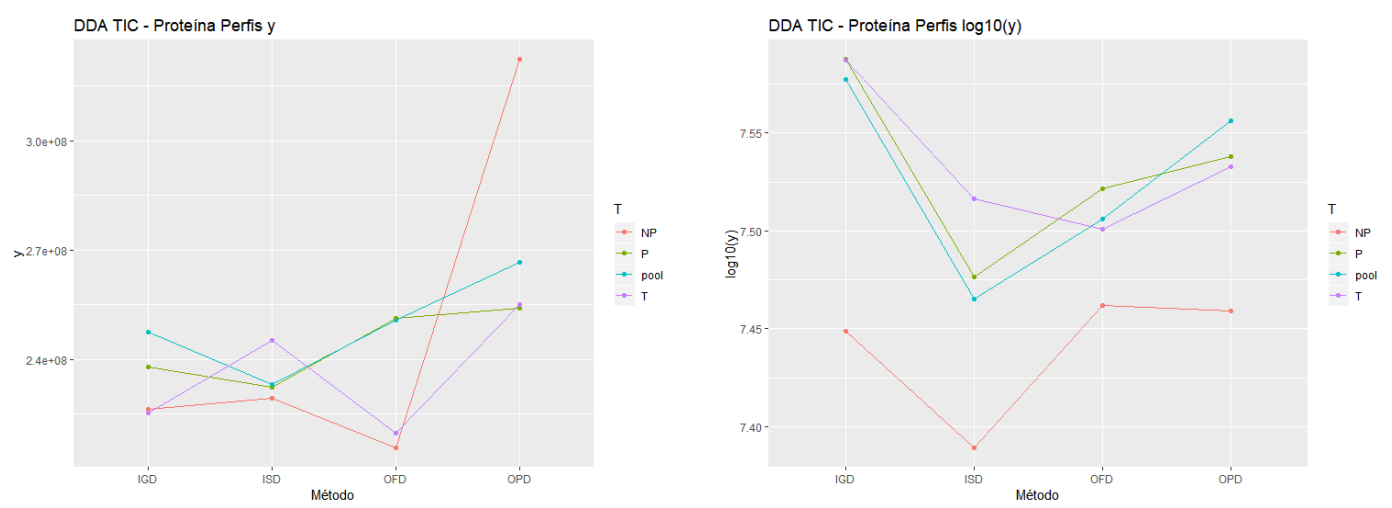


\subsubsection{Modelo de significância}

Para identificação das proteínas com comportamento diferencial foram gerados os gráficos vulcão apresentados na Figura 5.22 para todos os contrastes existentes entre a combinação dos fatores método dentro de tecido. Foram avaliadas 2156 das 3180 proteínas que compõem o recorte total quantificado pelo experimento, pois houve necessidade de retirar proteínas que não foram quantificadas em pelo menos uma das amostras para cada contraste de interesse.

Como resultado, 50 das 2156 proteínas avaliadas foram identificadas como significantes. De modo geral, foi observado que nos contrastes que comparam os métodos OFD e ISD foram identificadas o maior número de proteínas com comportamento diferencial significante. Assim como foi observado no experimento DDA noNorm, a proteína identificada pelo número 3126 foi considerada significante em quase todos os contrastes avaliados, com exceção do contraste que compara os métodos IGD e ISD em todos os tecidos. No geral, têm-se que das 50 proteínas com comportamento diferencial, 15 só foram consideradas significantes em um contraste, 16 em dois e 10 em três.

O grupo de proteínas identificadas como significantes nos experimentos DDA noNorm e DDA TIC não é exatamente o mesmo, as proteínas identificadas pelos números 36, 1535, 2908, 3377 e 1339 foram consideradas com comportamento diferencial somente para o experimento DDA TIC. Sendo que para o experimento DDA noNorm apresentado na seção anterior, as proteínas 12651, 2328, 2983, 2172, 2369, 2852, 532 e 1404 são significantes somente para o experimento DDA noNorm. Dessa forma, 45 proteínas foram consideradas com comportamento diferencial em ambos os experimentos, porém não necessariamente nos mesmos contrastes. 
Figura 5.22 - Gráficos vulcão para todos os contrastes entre pares de Métodos dentro de Tecido. Indicação de peptídeos significantes em vermelho ( $p$-valor $<$ $p$-valor ajustado e $\mid$ magnitude de mudança $\mid>2$ ))
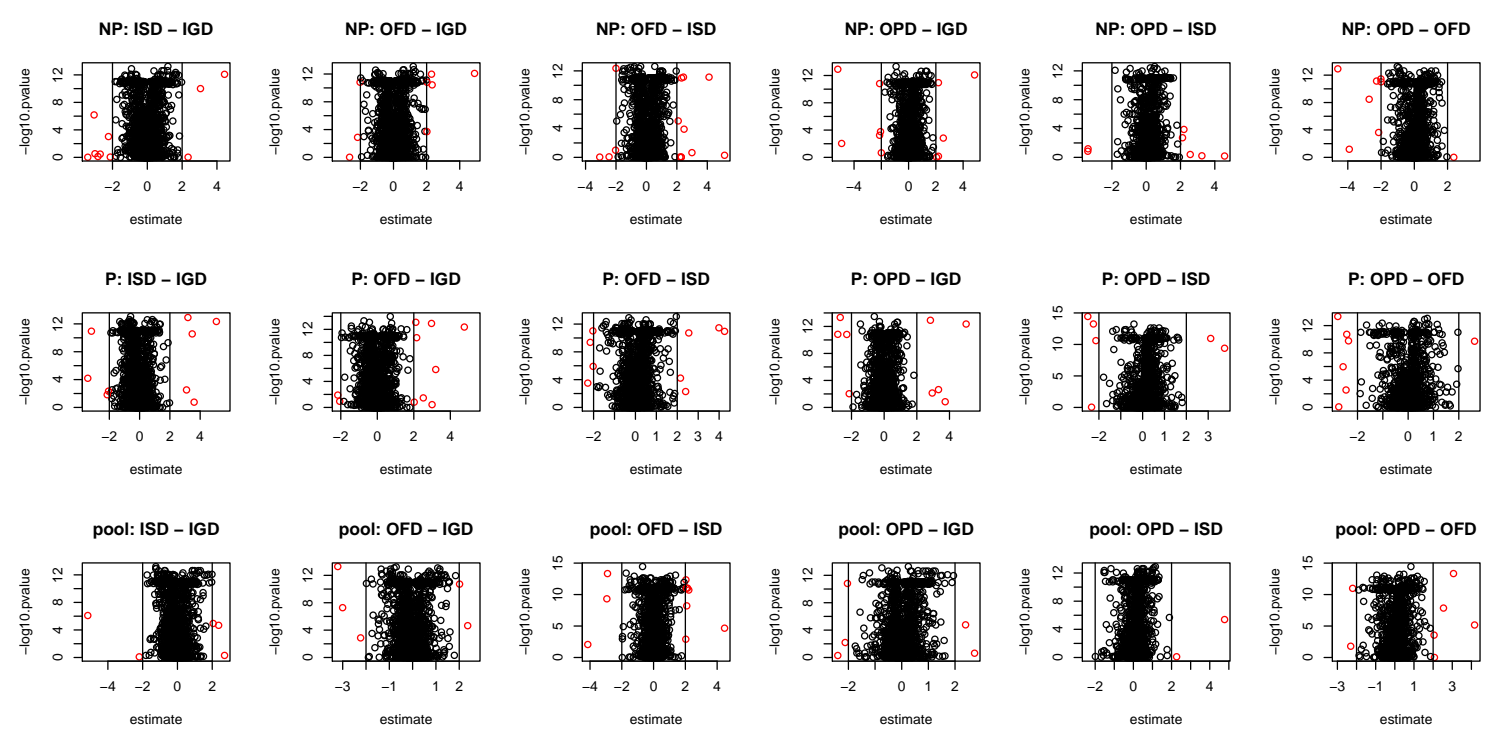

T: ISD - IGD

T: OFD - IGD
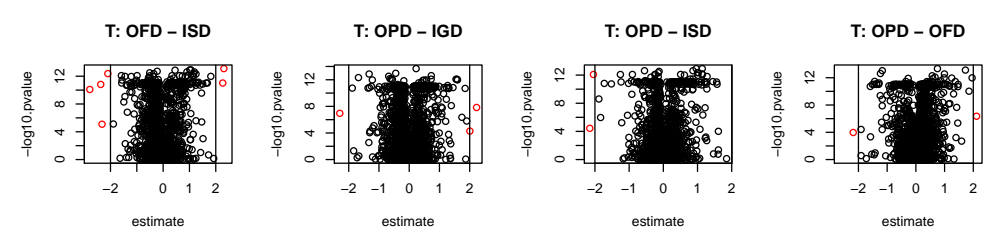


\subsection{Análise dos experimentos}

Pelos resultados obtidos em cada experimento avaliado foi observado que o método IGD possui as menores quantificações nos experimentos SRM e DDA noNorm, porém com os dados normalizados pela técnica proposta neste trabalho, notou-se que os diferentes métodos utilizados nos experimentos tiveram suas quantificações corrigidas, de tal maneira que passaram a apresentar comportamentos semelhantes entre si. Todavia, a normalização realizada nos experimentos DDA pela técnica TIC não conseguiu retirar o efeito da estrutura do delineamento presente nos dados. Mesmo após realizar o processo de normalização, as quantificações entre os métodos não ficaram tão similares, especialmente quanto às quantificações do método IGD que, após a normalização, eram as maiores entre os demais métodos avaliados.

No demais, observou-se que parte das proteínas e peptídeos identificados com comportamento diferencial na análise de significância é comum entre os experimentos noNorm e TIC, porém não necessariamente identificadas nos mesmos contrastes. Além disso, com base na normalização proposta neste trabalho foi possível notar que as quantificações das proteínas e peptídeos foram ajustadas, retirando a variação sistemática devido ao delineamento dos experimentos.

Portanto, com base no que foi apresentado tem-se que a metodologia proposta neste trabalho, tanto para normalização quanto para análise de significância, se mostraram como boas técnicas que podem ser utilizadas para retirar variação sistemática dos dados e para identificar variáveis com comportamento diferencial entre contrastes de interesse. Além disso, notou-se que a técnica de normalização influencia bastante nos resultados obtidos na análise de significância, sinalizando a importância de se escolher uma metodologia eficaz para realização da normalização dos dados. 


\subsection{Análises Multivariadas}

Nesta seção serão apresentados os resultados de duas técnicas bastante utilizadas para avaliar a estrutura de dados, a Análise de Componentes Principais e a Análise de Grafos Probabilísticos. Para os experimentos DDA noNorm (normalização dada pela metodologia proposta neste trabalho) e SRM foram considerados os resíduos padronizados do modelo de normalização estimado. Enquanto que para os experimentos DDA TIC só foi realizada a padronização das quantificações já normalizadas previamente pela técnica TIC. Vale mencionar que as análises foram realizadas somente sobre os dados completos, isto é, foram consideradas somente as variáveis quantificadas em todas as 80 amostras. Com base na técnica de decomposição de dados ASCA apresentada na Seção 4.4, os resíduos padronizados e não correlacionados foram decompostos segundo as componentes de variação correspondentes aos efeitos avaliados no estudo, dando foco ao efeito do método dentro do tecido. Dessa forma, considerando a matriz relacionada ao efeito de interesse foram geradas componentes principais e grafos probabilísticos para representar e melhor entender a estrutura dos dados avaliados. Os resultados obtidos estão apresentados a seguir para cada recorte avaliado.

\subsubsection{Análise de Componentes Principais}

As componentes principais obtidas para o experimento SRM estão apresentadas na Figura 5.23. Nota-se pela representação do efeito método dentro de tecido que o tecido Pool, que representa uma combinação de todos os tecidos avaliados, possui uma maior variabilidade, destacando-se em relação aos demais. Além disso, pela representação dos peptídeos quantificados é possível perceber que alguns peptídeos (1 - IPVGPETLGR; 14 - FGFYEVFK; 15 - AAYFGIYDTAK; 16 - AIWNVINWENVTER) possuem uma maior variabilidade e acabam se destacando no gráfico apresentado, porém grande parte dos peptídeos estão concentrados na região em que as duas componentes possuem valor nulo.

Figura 5.23 - Análise de Componentes Principais - Experimento SRM

(a) SRM: Representação do efeito aninhado $\mathrm{M}(\mathrm{T})$

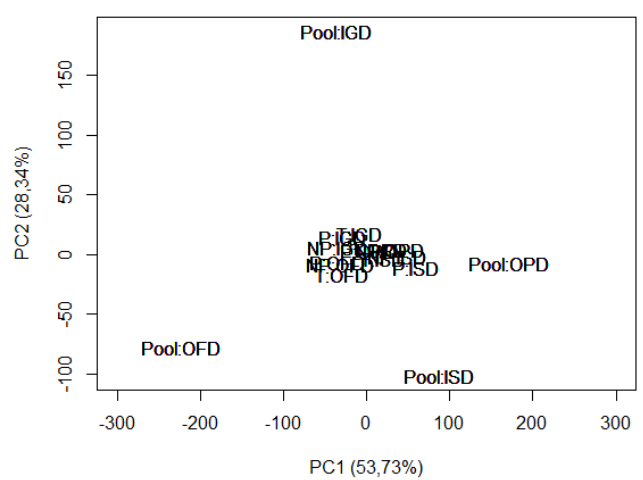

(b) SRM: Representação dos peptídeos quantificados

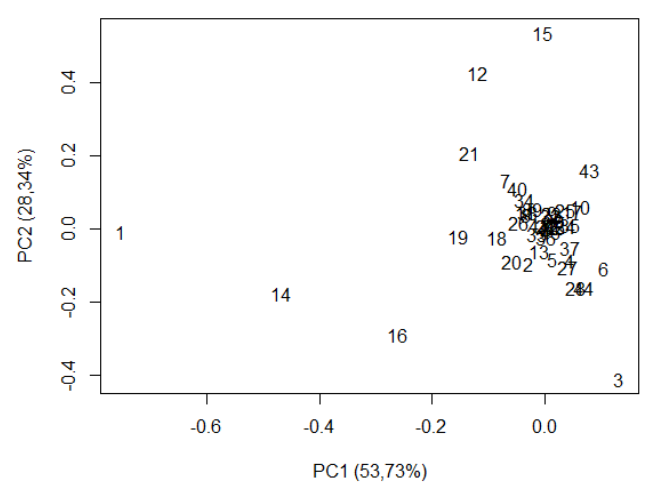


Nas Figuras 5.24 e 5.25 estão apresentados os resultados obtidos pela Análise de Componentes Principais para o experimento DDA - Peptídeos considerando os dados normalizados pela metodologia proposta neste trabalho e pela técnica TIC. Nota-se que os gráficos gerados são bem distintos, a representação do efeito do método dentro de tecido, para os dados baseados no modelo de normalização, destaca a discrepância existente entre os tecidos Pool e NP em relação aos demais. Enquanto que para os dados baseados na técnica TIC o destaque fica somente em torno do tecido Pool.

Em relação à representação dos peptídeos identificados tem-se que grande parte está concentrada na região próxima do zero em ambas as componentes. Porém nota-se que para os dados normalizados pelo modelo estatístico, o peptídeo AVTEQGHELSNEER (4821) se destaca dos demais.

Figura 5.24 - Análise de Componentes Principais - Experimento DDA noNorm Peptídeos

(a) DDA noNorm: Representação do efeito aninhado $\mathrm{M}(\mathrm{T})$

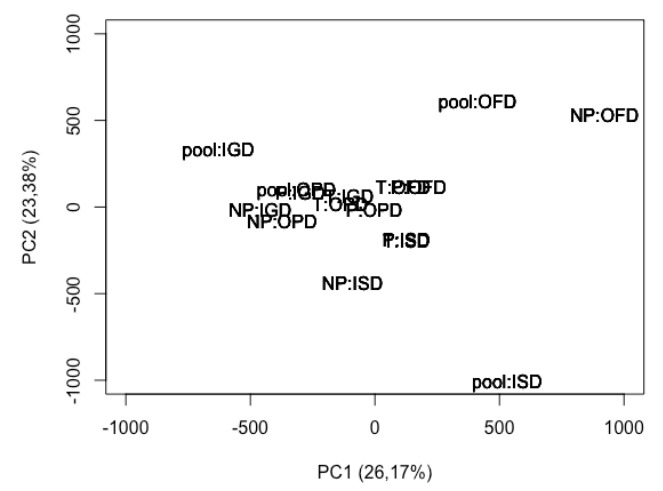

(b) DDA noNorm: Representação dos peptídeos identificados

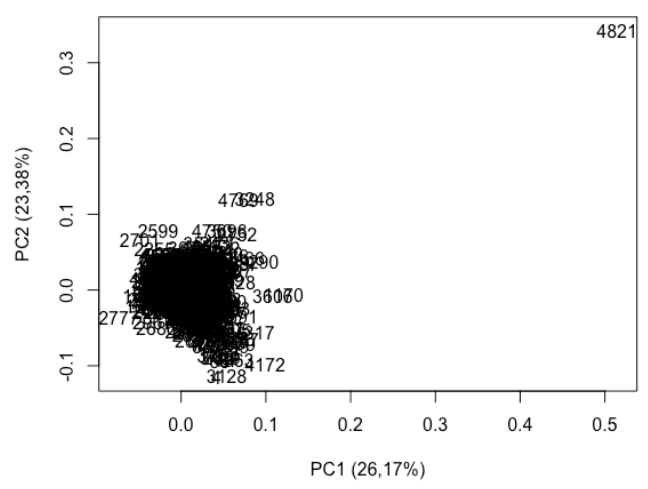

Por fim, nas Figuras 5.26 e 5.27 estão apresentados os gráficos com as componentes principais obtidas para os experimentos DDA - Proteínas (noNorm e TIC). Para os dados normalizados pela metodologia descrita neste trabalho destaca-se que na primeira componente somente o tecido NP apresenta comportamento distinto dos demais. No entanto, para a segunda componente o tecido Pool é o que maior se destaca dos demais.

Agora para os dados normalizados pela técnica TIC, observa-se um comportamento semelhante ao que foi encontrado para o experimento DDA - Peptídeos (TIC), em que o tecido Pool se destaca dos demais tecidos avaliados.

Em relação à representação das proteínas, tem-se que (Figura 5.26) grande parte está concentrada próximo do zero na primeira componente, com exceção para a proteína 12461. No entanto para os dados obtidos pela técnica TIC (Figura 5.27) observa-se que as proteínas no geral estão concentradas próxima do zero em ambas as componentes, com pequenos desvios (proteínas 595 e 2163).

No geral, foram observadas poucas variáveis (peptídeos ou proteínas) com comportamento bem distante dos demais. Em relação à representação do efeito do método dentro de tecido, somente os tecidos Pool e NP tiveram um destaque a depender 
Figura 5.25 - Análise de Componentes Principais - Experimento DDA TIC Peptídeos

(a) DDA TIC: Representação do efeito aninhado $\mathrm{M}(\mathrm{T})$

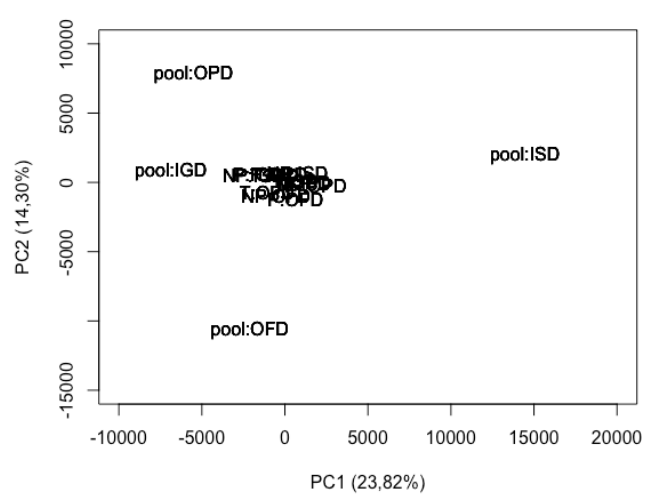

(b) DDA TIC: Representação dos peptídeos identificados

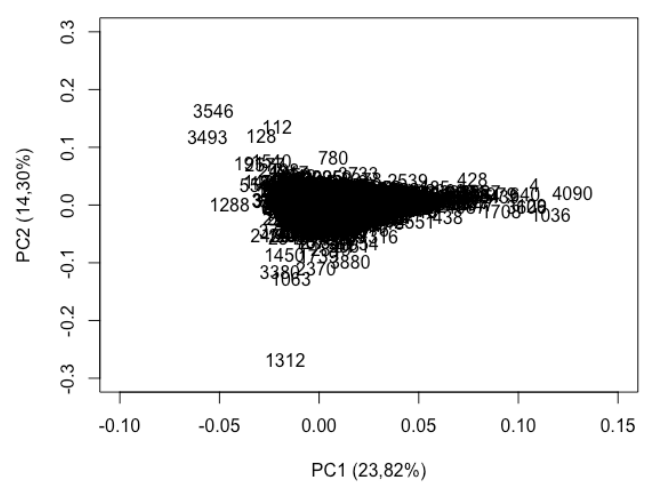

Figura 5.26 - Análise de Componentes Principais - Experimento DDA noNorm Proteínas

(a) DDA noNorm: Representação do efeito aninhado $\mathrm{M}(\mathrm{T})$

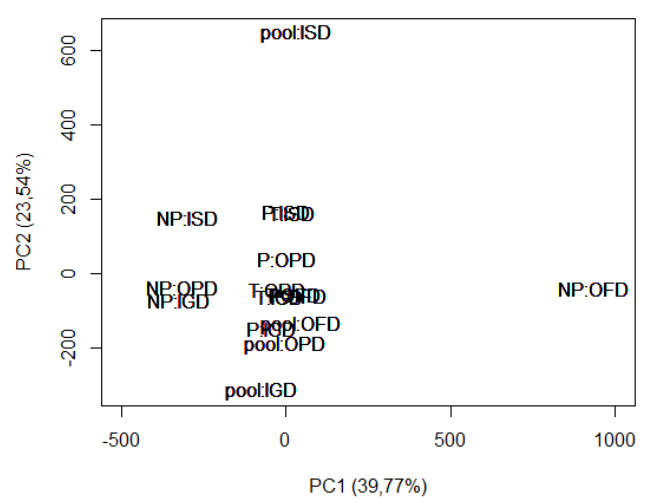

(b) DDA noNorm: Representação das proteínas identificadas

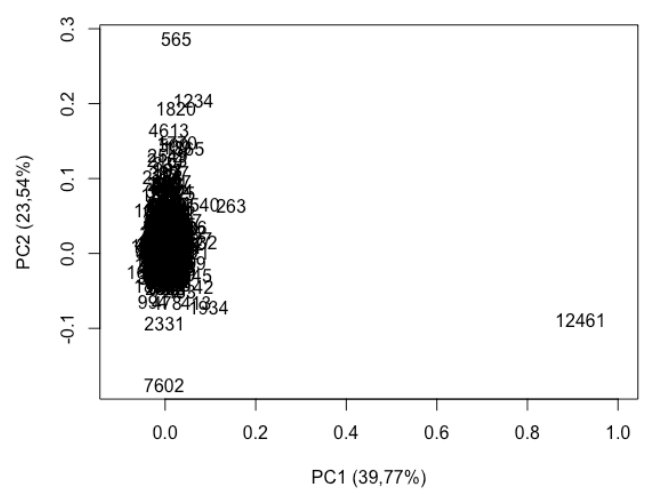


Figura 5.27 - Análise de Componentes Principais - Experimento DDA TIC Proteínas

(a) DDA TIC: Representação do efeito aninhado $\mathrm{M}(\mathrm{T})$

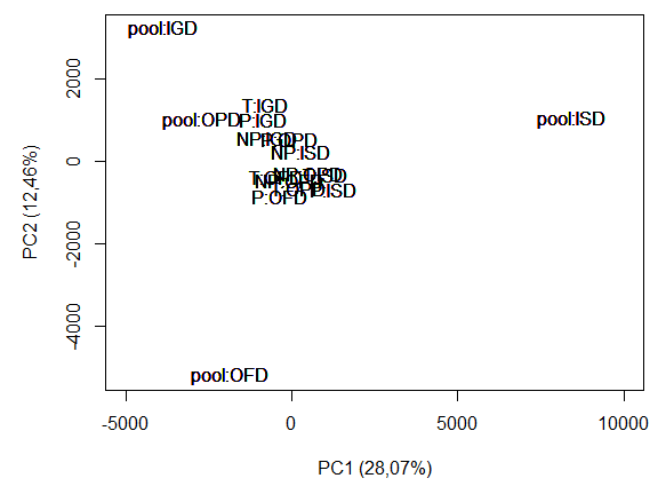

(b) DDA TIC: Representação das proteínas identificadas

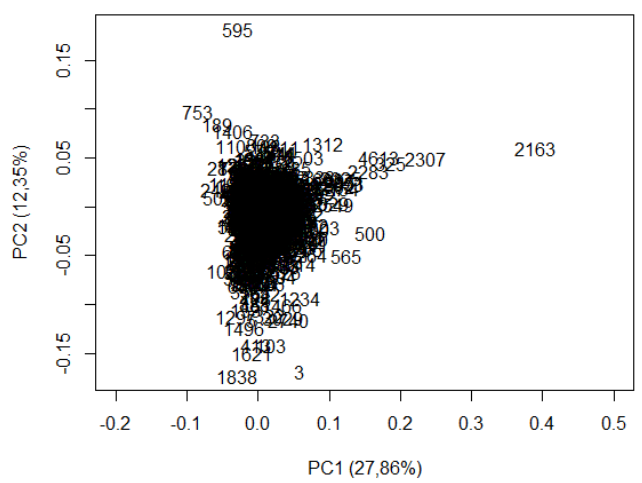

ainda do experimento avaliado.

Vale mencionar que a descrição dos peptídeos apresentados nas análises multivariadas estão apresentados no Apêndice, podendo ser consultados quando se achar necessário. 


\subsubsection{Análise de Grafos Probabilísticos}

Nesta seção serão apresentados os resultados obtidos pela Análise de Grafos Probabilísticos com o intuito de se iniciar o estudo da estrutura de causalidade entre as variáveis (peptídeos ou proteínas) quantificadas nos diferentes experimentos. Vale ressaltar que os resultados aqui expostos são de caráter descritivo, obtidos com o intuito de exemplificar a utilização da técnica em estudos proteômicos. Os resultados foram obtidos por meio dos pacotes pcalg e igraph presentes no software estatístico R. $[16,12,5]$

Nas Figuras 5.28 e 5.29 estão apresentados os dois maiores grafos conexos obtidos para o experimento SRM, compostos por 33 e 8 peptídeos respectivamente. Vale mencionar que o recorte utilizado para geração dos grafos considerou somente os peptídeos quantificados em todas as (80) amostras, avaliando assim 46 do total de 68 peptídeos quantificados pelo experimento originalmente.

Nota-se que os grafos obtidos possuem setas bi-direcionadas, que implica que o algoritmo utilizado não conseguiu prever, de forma única, a real estrutura de relação entre os peptídeos avaliados.

Além dos dois grafos apresentados nas Figuras 5.28 e 5.29, foi observado ainda que o peptídeo IIDAALR (3) é marginalmente independente dos demais e os peptídeos IVFSPEEAK (34) e ATAEQISSQTGNK (39) estão correlacionados entre si, porém não foi possível identificar a direção dessa associação. Esse mesmo fato foi observado entre os peptídeos DFTATDLSEFAAK (37) e DGPGFYTTR (45).

Vale ressaltar que a descrição dos peptídeos apresentados nas análises multivariadas estão apresentados no Apêndice, podendo ser consultados em detalhe. 
Figura 5.28 - Maior grafo conexo estimado para o experimento SRM: Grafo1-SRM

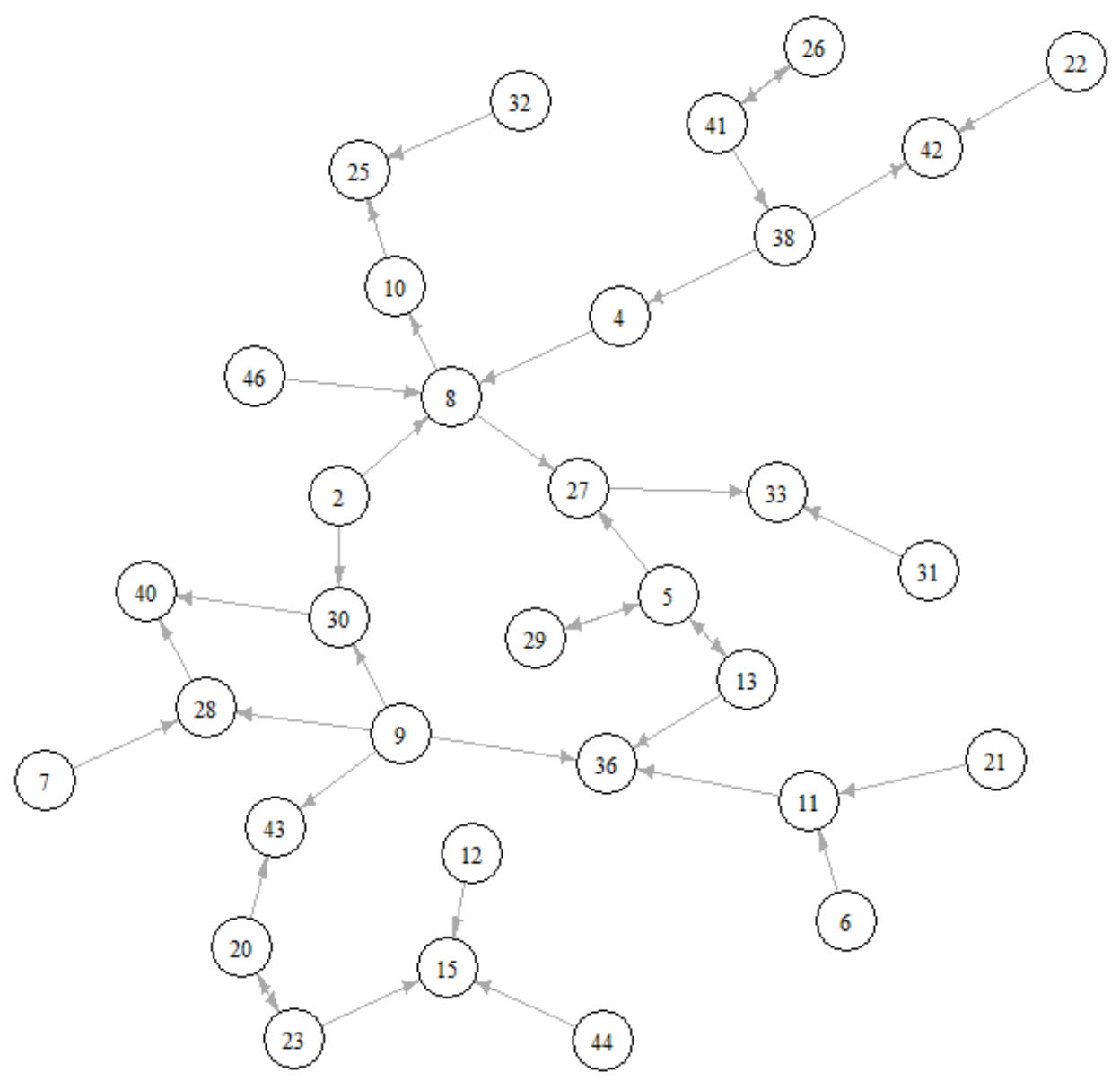


Figura 5.29 - Segundo maior grafo conexo estimado para o experimento SRM:

Grafo2-SRM

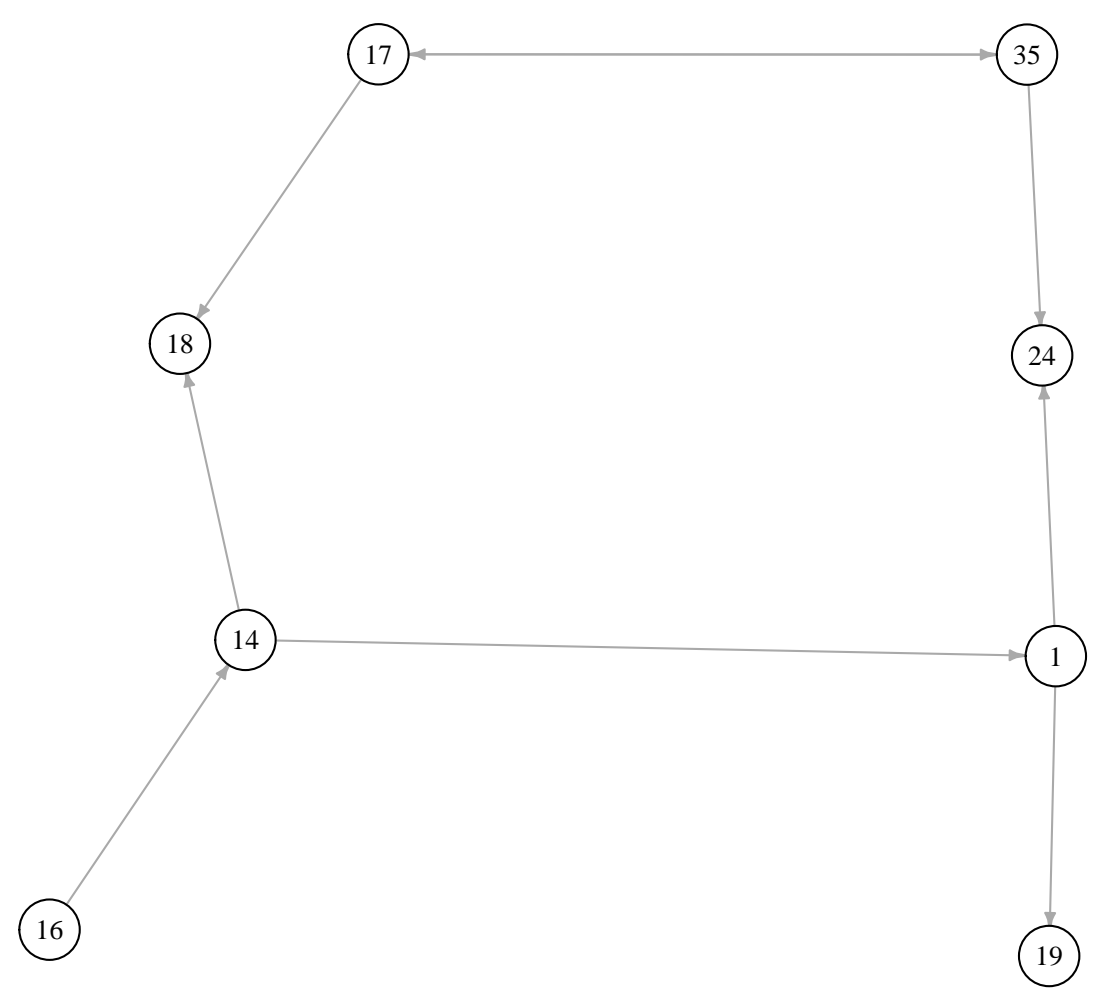


Considerando agora o experimento DDA - Peptídeos, o recorte utilizado para estimação da estrutura de causalidade entre os peptídeos considerou 4824 dos 37485 peptídeos identificados em ambos os experimento noNorm e TIC, para obtenção desse recorte foram considerados somente os peptídeos quantificados em todas as amostras avaliadas.

Para os dados normalizados com base na metodologia apresentada neste trabalho (noNorm) foram identificados 137 grafos conexos compostos por pelo menos quatro peptídeos. Identificou-se ainda que 2670 dos 4824 peptídeos avaliados foram considerados marginalmente independentes. Nas Figuras 5.30 e 5.31 estão apresentados os maiores grafos conexos estimados, contendo entre 12 à 17 peptídeos.

Figura 5.30 - Maior grafo conexo estimado para o experimento DDA noNorm Peptídeos: Grafo 1 - DDA noNorm

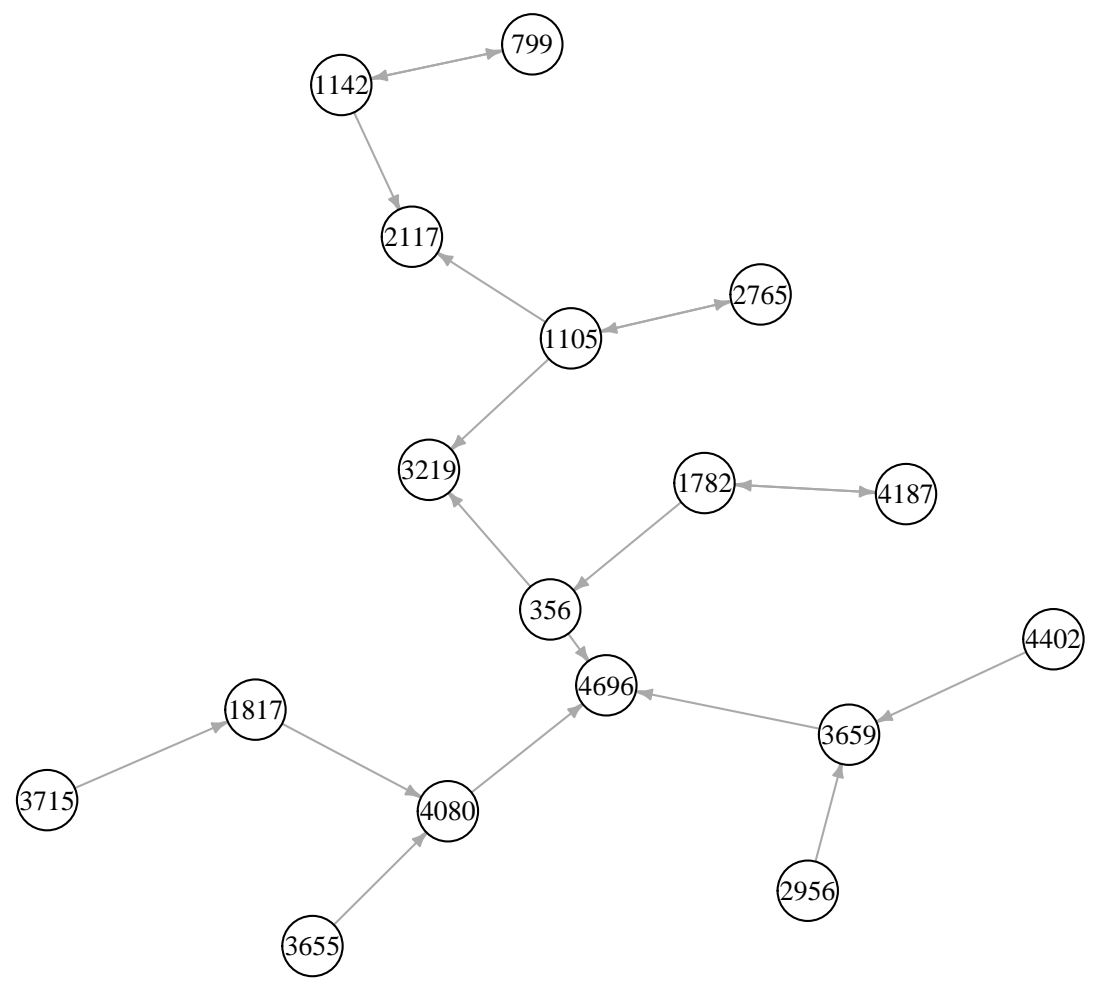


Figura 5.31 - Grafos conexos estimados com o maior número de peptídeos para o experimento DDA noNorm - Peptídeos

(a) Grafo 2 - DDA noNorm Peptídeos

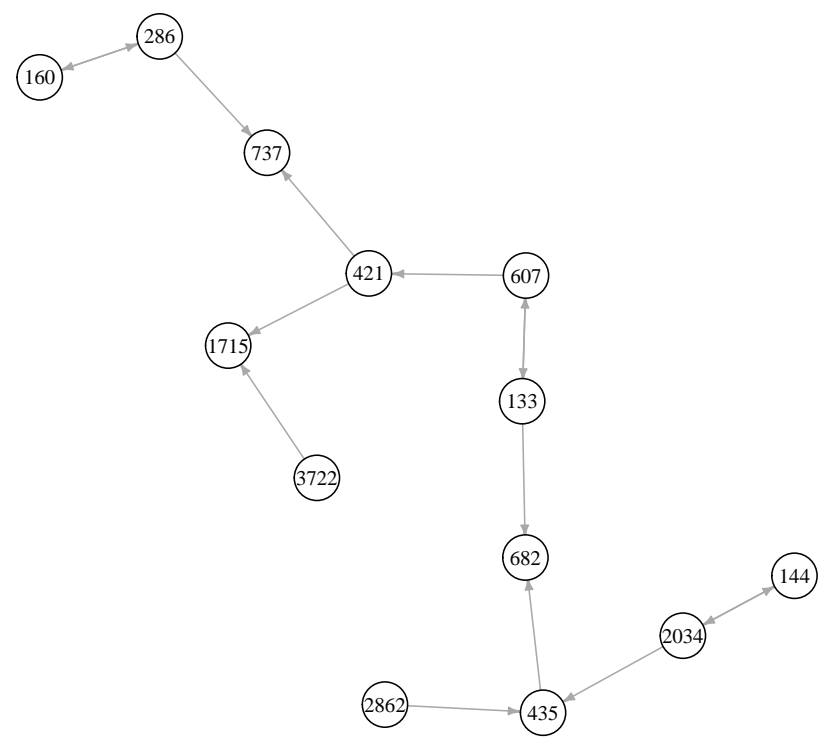

(b) Grafo 3 - DDA noNorm Peptídeos

(c) Grafo 4 - DDA noNorm Peptídeos
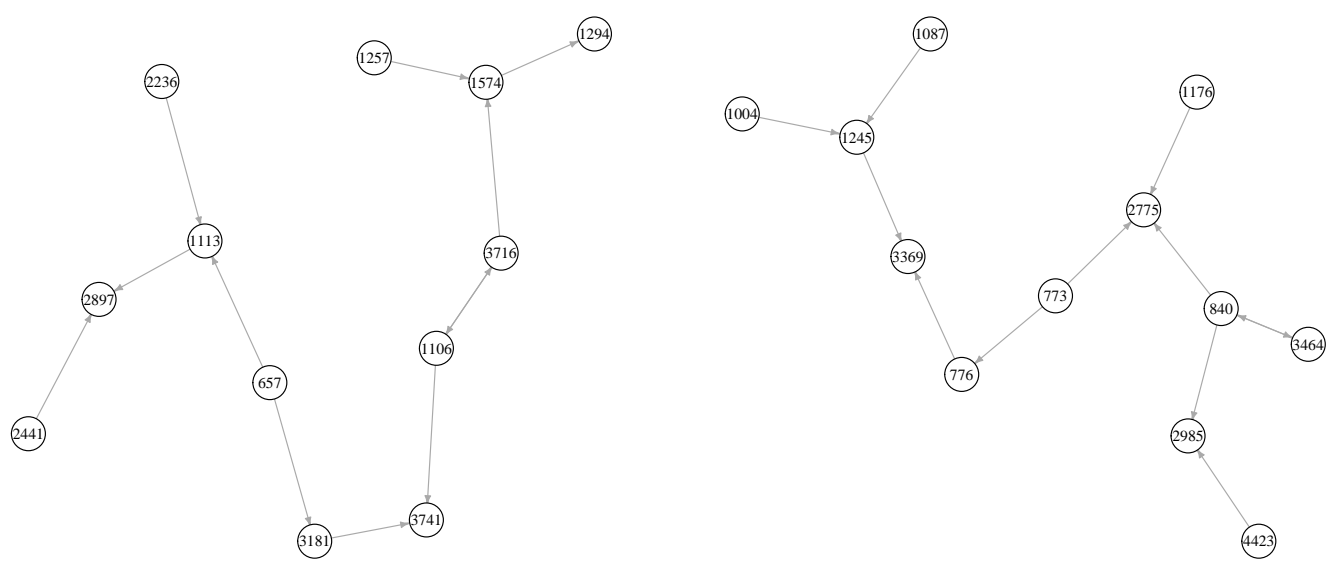
Para o experimento DDA - Peptídeos com normalização baseada na técnica TIC foram estimados 100 grafos conexos contendo pelo menos quatro peptídeos e identificouse 2845 peptídeos como marginalmente independentes. Nas Figuras 5.32 e 5.33 estão apresentados os maiores grafos conexos estimados, contendo entre 10 à 12 peptídeos.

Comparando os resultados obtidos nos experimentos noNorm e TIC observa-se que a estrutura de causalidade estimada entre os recortes não é igual. Sendo que as dissimilaridades entre os resultados podem ser observadas desde no número de peptídeos marginalmente independentes quanto nos principais grafos estimados, enfatizando que dependendo da técnica de normalização escolhida os resultados obtidos podem ser bem distintos.

Figura 5.32 - Maior grafo conexo estimado para o experimento DDA TIC Peptídeos: Grafo 1 - DDA TIC

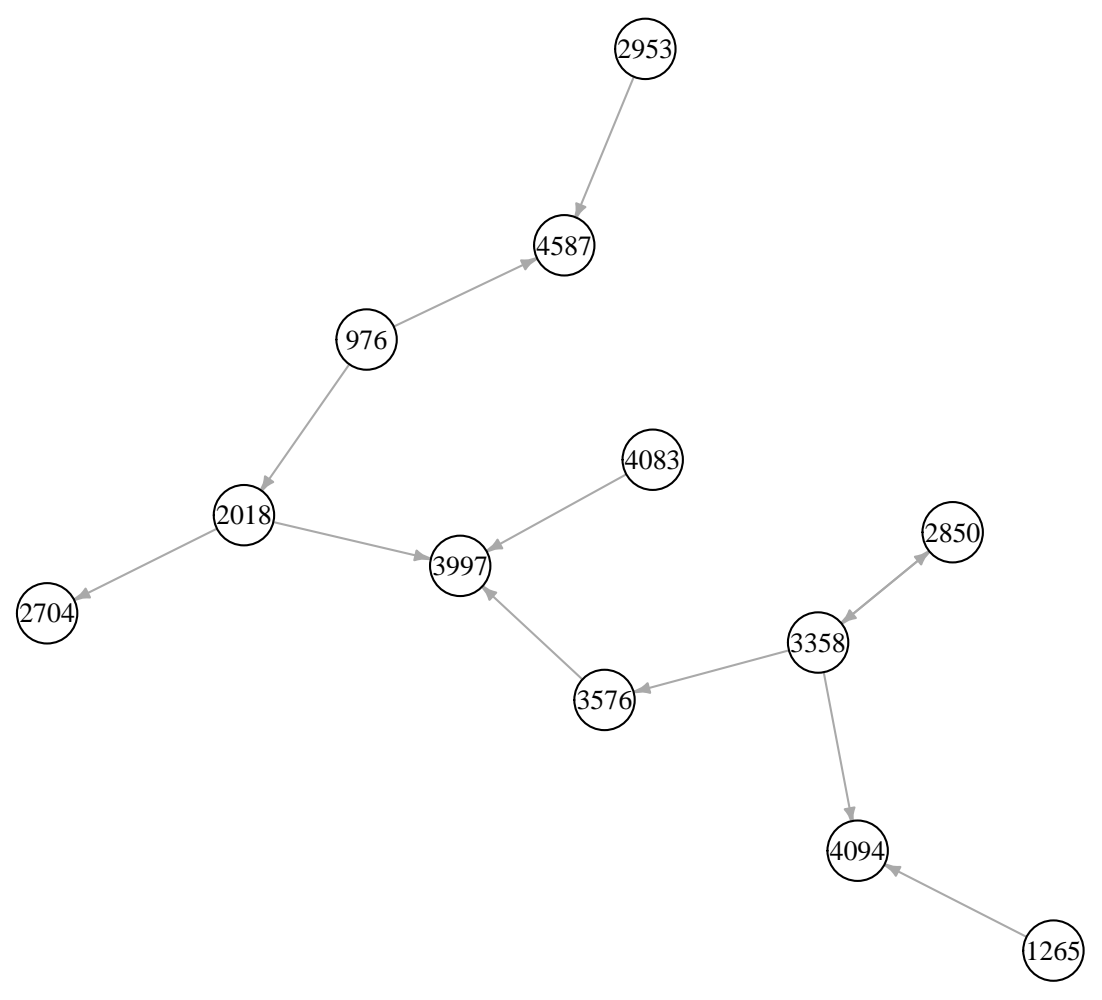


Figura 5.33 - Grafos conexos estimados com o maior número de peptídeos para o experimento DDA TIC - Peptídeos

(a) Grafo 2 - DDA TIC Peptídeos

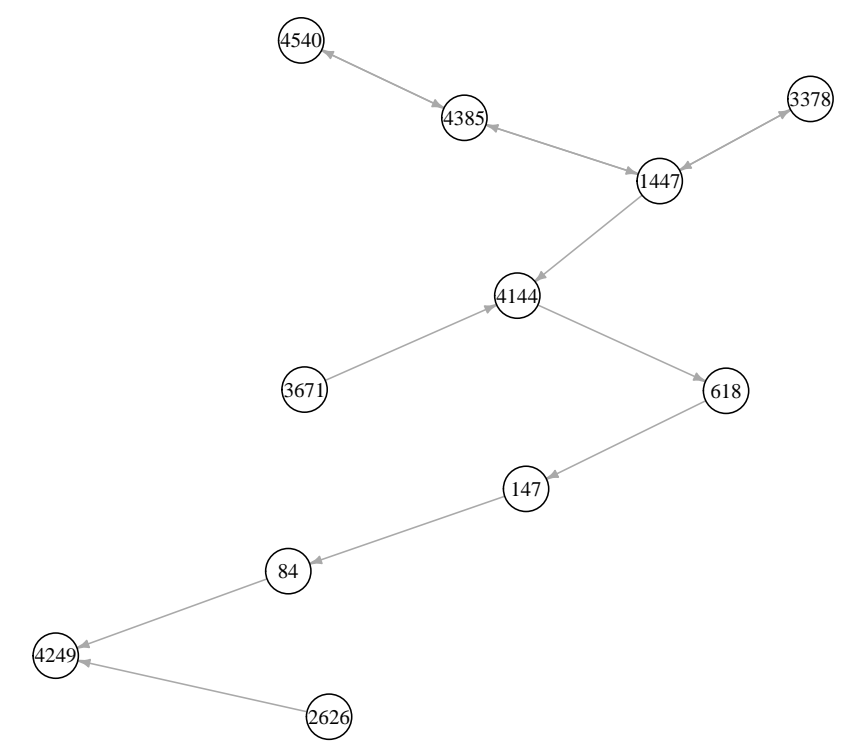

(b) Grafo 3 - DDA TIC Peptídeos

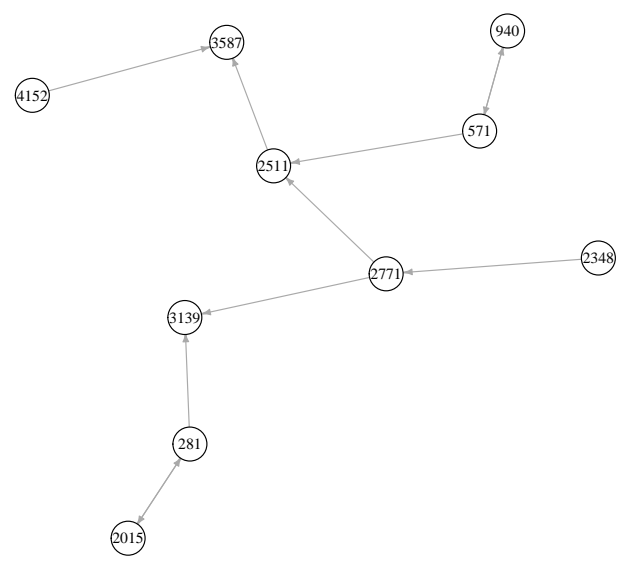


Agora, com base nos dados obtidos pelo experimento DDA Proteínas, foram estimados grafos probabilísticos para avaliar a estrutura de causalidade entre as variáveis (proteínas) quantificadas nos experimentos DDA noNorm e TIC. Em ambos os experimentos foram avaliadas somente as proteínas quantificadas em todas as amostras, resultando em um recorte com 1259 proteínas das 3306 quantificadas para o experimento DDA noNorm. As quantificações dessas proteínas foram normalizadas considerando o modelo proposto neste trabalho e por meio da estimação da estrutura de causalidade dessas proteínas foram obtidos 64 grafos conexos contendo pelo menos quatro proteínas. Além disso, observou-se que das 1259 proteínas avaliadas 454 foram identificadas como marginalmente independentes.

Nas Figuras 5.34 e 5.35 estão apresentados os grafos conexos com maior número de proteínas, contendo entre 19 à 29 proteínas.

Figura 5.34 - Maior grafo conexo estimado para o experimento DDA noNorm Proteínas: Grafo 1 - DDA noNorm

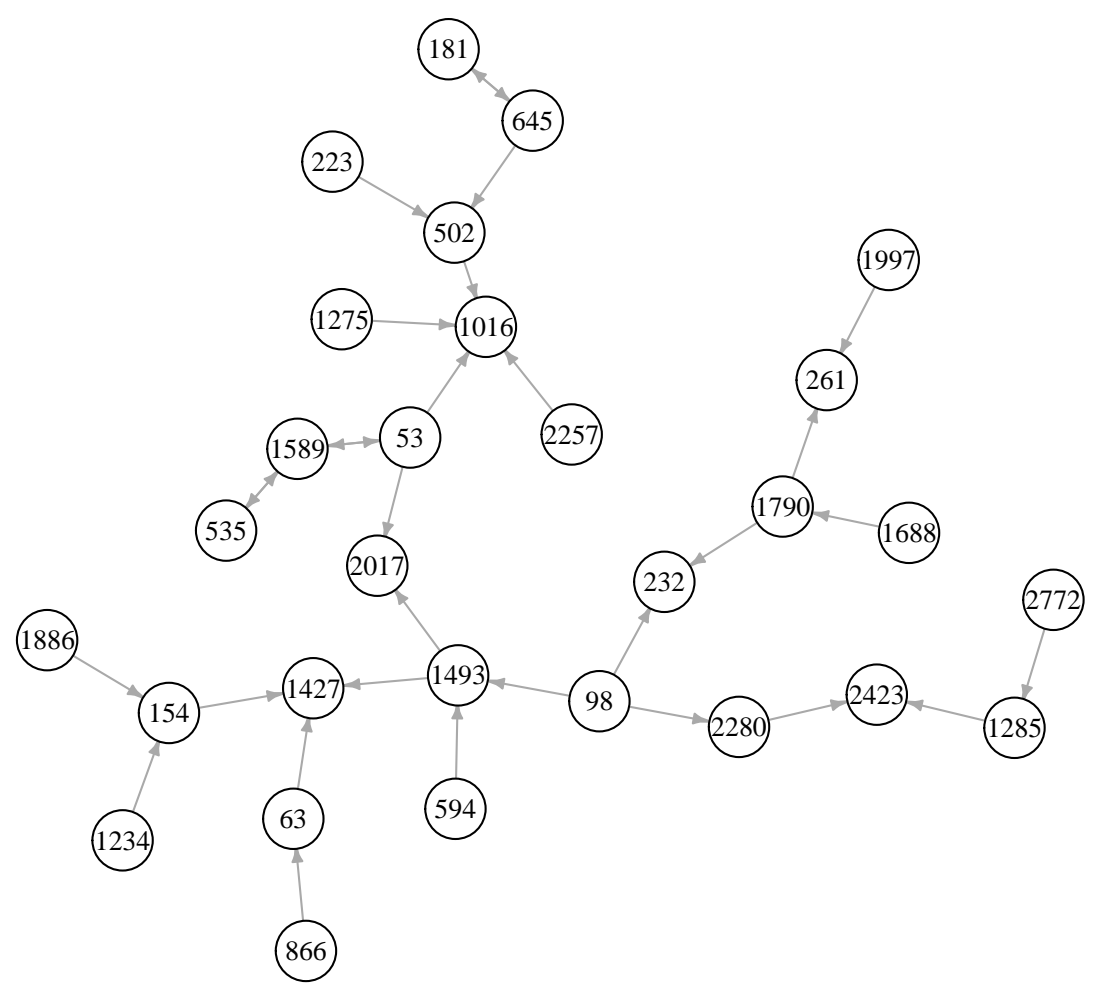


Figura 5.35 - Grafos conexos estimados com o maior número de proteínas para o experimento DDA noNorm - Proteínas

(a) Grafo 2 - DDA noNorm Proteínas

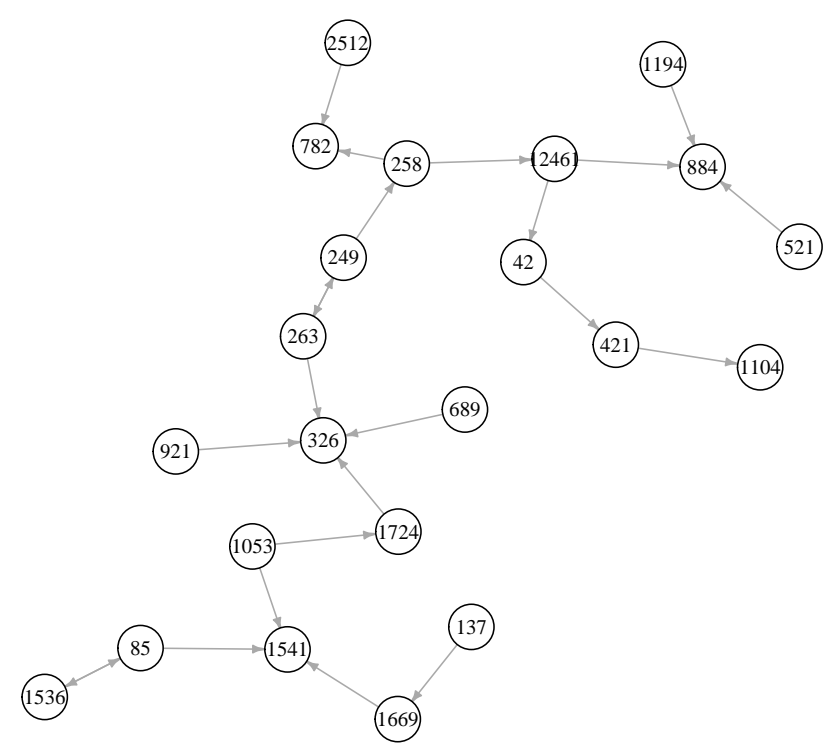

(b) Grafo 3 - DDA noNorm Proteínas

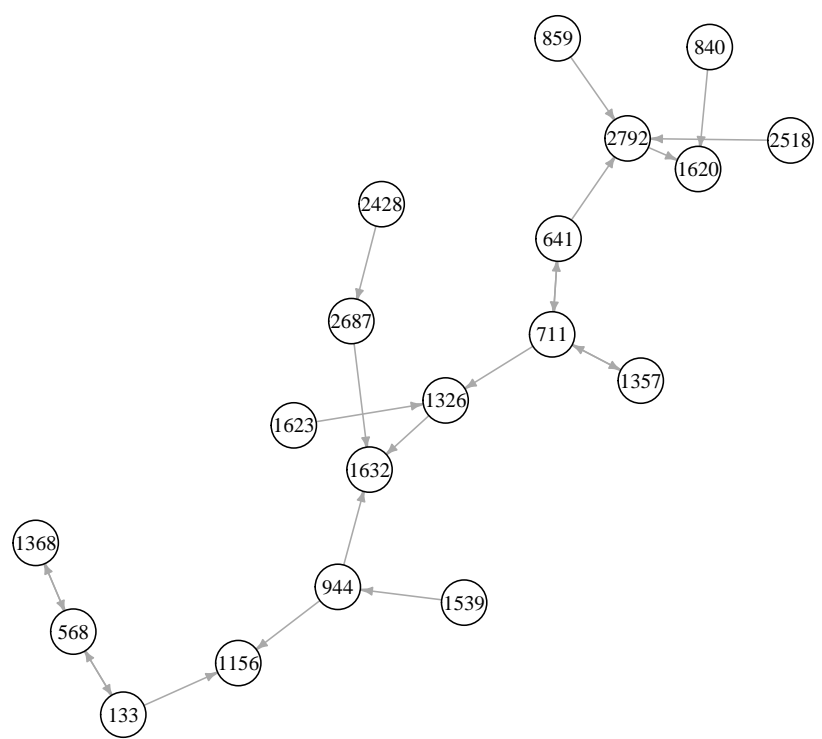


Por fim, considerando os dados referentes ao experimento DDA - Proteínas com normalização dada pela técnica TIC foram estimados 56 grafos conexos contendo pelo menos quatro proteínas. Neste experimento, foram avaliadas 1160 proteínas quantificadas em todas as amostras avaliadas, das quais 462 foram identificadas como marginalmente independentes. Nas Figuras 5.36, 5.37 e 5.38 estão apresentados os maiores grafos conexos estimados para o experimento, contendo entre 26 à 31 proteínas.

Figura 5.36 - Maiores grafos conexos estimados para o experimento DDA TIC Proteínas - Grafo 1 - DDA TIC Proteínas

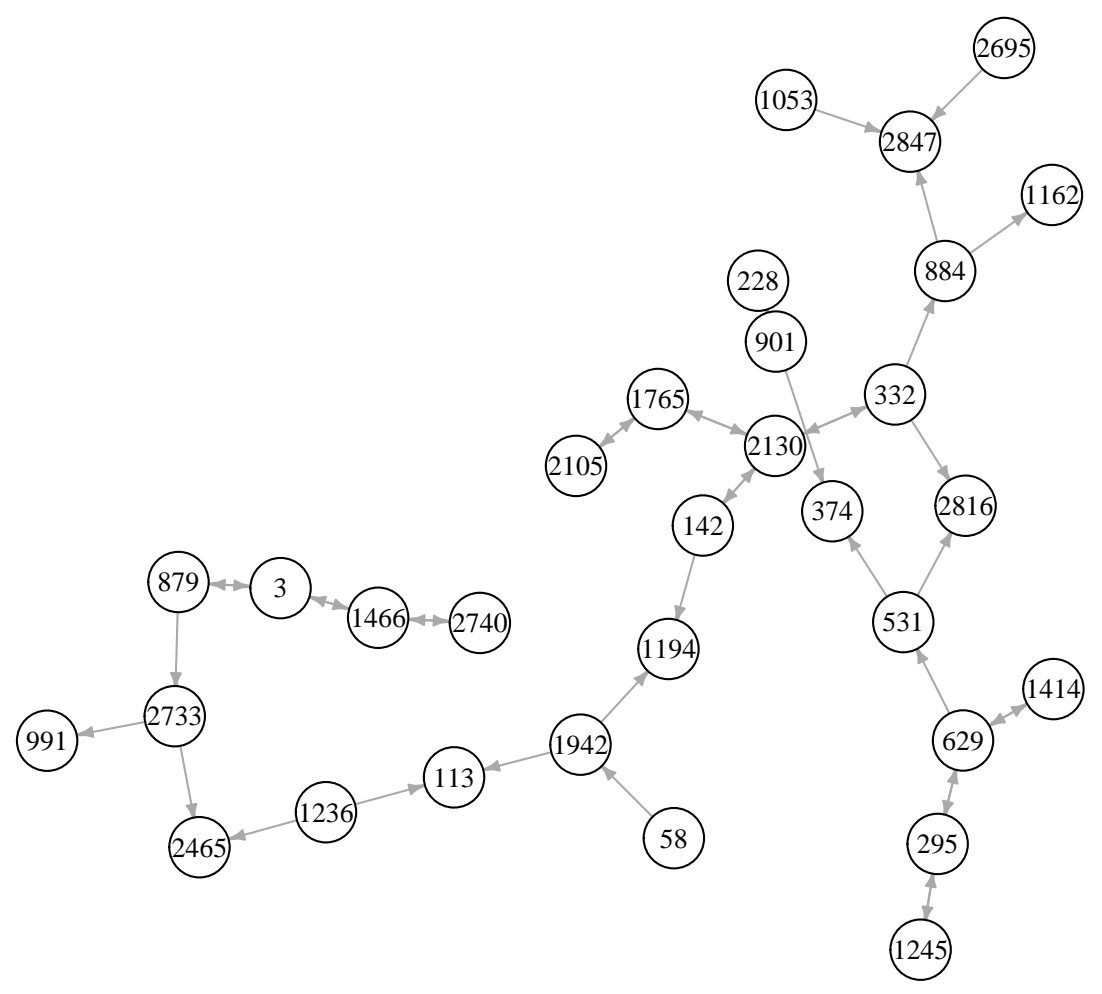

No geral, grande parte dos grafos conexos obtidos nos experimentos são compostos por uma baixa quantidade de peptídeos e ou proteínas. Todavia, com base nos resultados obtidos é possível ter um melhor entendimento a respeito da estrutura causal existente entre as variáveis avaliadas. Vale mencionar que não foi possível estimar de forma única a verdadeira estrutura de causalidade entre todos os peptídeos e ou proteínas avaliadas.

Além disso, foi possível perceber que o modelo de normalização escolhido in- 
Figura 5.37 - Maiores grafos conexos estimados para o experimento DDA TIC Proteínas - Grafo 2 - DDA TIC Proteínas

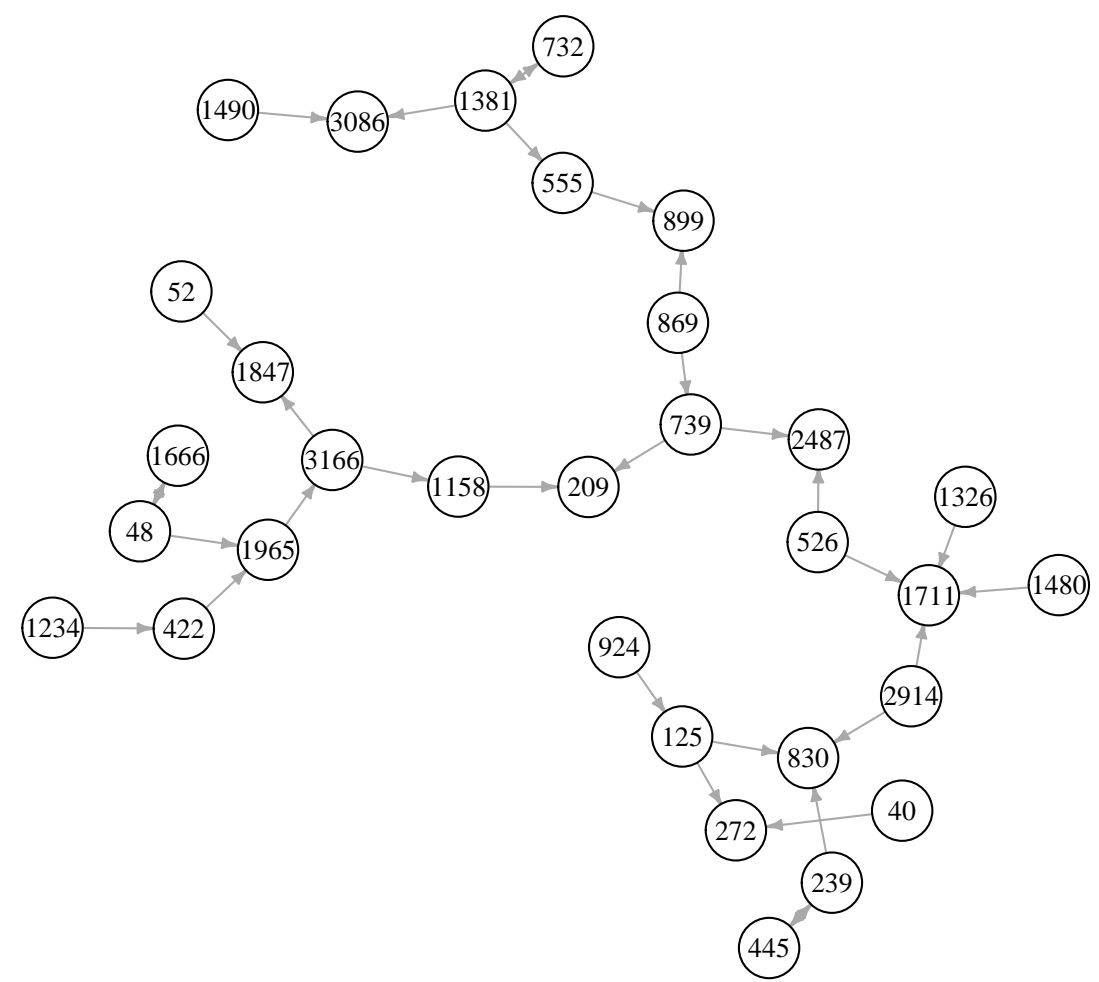


Figura 5.38 - Maiores grafos conexos estimados para o experimento DDA TIC Proteínas - Grafo 3 - DDA TIC Proteínas

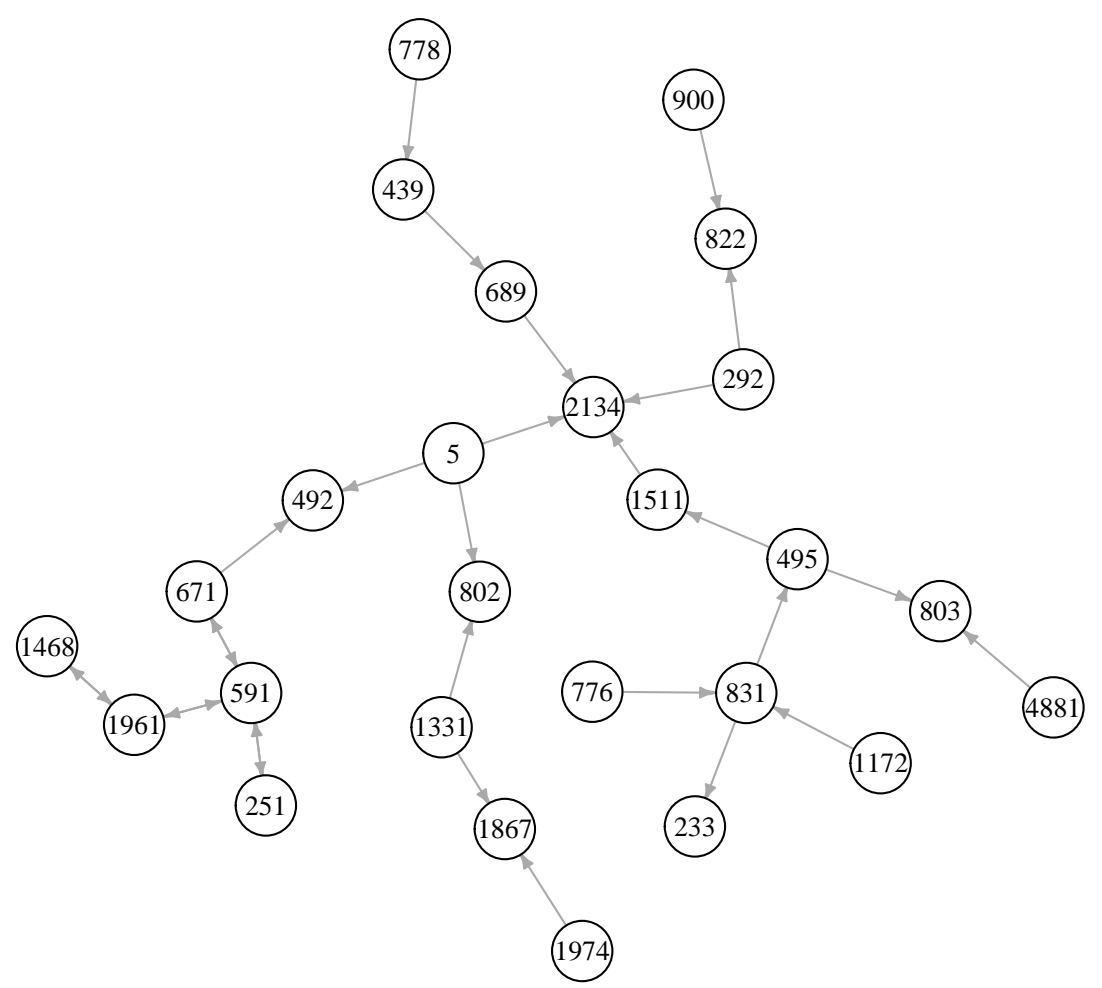


fluencia nos resultados obtidos. Portanto, a depender da metodologia utilizada as conclusões obtidas podem ser bastante diferentes, como foi observado neste trabalho. 


\section{Capítulo 6}

\section{Considerações Finais}

Com base nos resultados obtidos pela metodologia de normalização proposta neste trabalho foi possível identificar que no geral o método de preparação em gel (IGD) apresentou as menores quantificações entre os métodos avaliados e o método em solução (ISD) foi considerado o mais preciso dentro dos tecidos estudados nos experimentos. Além disso, foi possível identificar as diferentes fontes de variação impostas pelo experimento e considerá-las nos modelos de normalização e significância.

Notou-se ainda que, por meio da utilização dos resíduos condicionais do modelo de normalização foi possível retirar variações sistemáticas devido ao delineamento do experimento, possibilitando avaliar a significância, estrutura dos dados e causalidade entre os peptídeos e proteínas quantificadas nos experimentos com base principalmente na variação biológica presente nos dados.

A análise de significância proposta neste trabalho possibilitou identificar os peptídeos e proteínas com comportamento significantes entre diferentes condições de interesse. Como apoio visual, o gráfico vulcão se mostrou uma boa ferramenta para visualização e identificação de variáveis com comportamento diferencial.

Com base nos resíduos não correlacionados e padronizados do modelo de normalização foi possível ainda avaliar a estrutura dos dados em cada um dos experimentos por meio da análise de técnicas multivariadas, em que foi possível identificar os efeitos e variáveis com comportamento distinto entre as observações avaliadas, dando destaque para o tecido Pool e NP. Além disso, foi avaliada de forma descritiva a estrutura de causalidade presente entre os peptídeos e proteínas quantificadas nos diferentes experimentos. Nota-se que para os experimentos DDA (peptídeos e proteínas) grande parte das variáveis avaliadas não estão relacionadas entre si.

Comparando os resultados obtidos pelos experimentos noNorm e TIC foi possível perceber o quanto a técnica de normalização utilizada influencia os resultados obtidos na análise de significância, de componentes principais e grafos probabilísticos, enfatizando a importância de se utilizar técnicas eficazes e bem justificadas para obtenção de dados normalizados.

Para estudos futuros pode-se pensar em avaliar uma possível relação entre os peptídeos e proteínas quantificadas nos experimentos podendo dar maior suporte na validação dos resultados de significância bem como melhor entendimento de relações de causalidade encontradas entre as variáveis avaliadas. Além disso, pode ser de interesse ainda utilizar algum método de imputação flexível o bastante para atender 
o padrão de observações faltantes, para que sejam consideradas todas as amostras do experimento nas análises avaliadas neste trabalho, pois como foi possível perceber nas análises de significância e nas análises multivariadas teve-se necessidade de se retirar uma grande quantidade de variáveis que apresentaram dados incompletos. Por fim, pode-se pensar em aplicar técnicas esparsas para avaliar a estrutura dos dados, principalmente nos casos com grande quantidade de variáveis, como foi observado nos experimento DDA.

Em adição aos resultados os códigos utilizados para análise foram disponibilizados na URL github.com/acarolcruz/proteomics. 


\section{Referências Bibliográficas}

[1] Barbieri, R., Guryev, V., Brandsma, C.-A., Suits, F., Bischoff, R., And Horvatovich, P. Proteogenomics: Key Driver for Clinical Discovery and Personalized Medicine. Springer, 2016.

[2] Barbosa, E. B., Vidotto, A., Polachini, G. M., Henrique, T., de Marqui, A. B. T., And Tajara, E. H. Proteomics: methodologies and applications to the study of human diseases. Revista da Associação Médica Brasileira (English Edition) (2012).

[3] Bates, D., Mächler, M., Bolker, B., And Walker, S. Fitting linear mixed-effects models using lme4. Journal of Statistical Software (2015).

[4] Callister, S. J., Barry, R. C., Adkins, J. N., Johnson, E. T., Qian, W. J., Webb-Robertson, B. J., Smith, R. D., and Lipton, M. S. Normalization approaches for removing systematic biases associated with mass spectrometry and label-free proteomics. Journal of proteome research (2006).

[5] Csardi, G., And Nepusz, T. The igraph software package for complex network research. InterJournal Complex Systems, 1695 (2006).

[6] Demidenko, E. Mixed Models: Theory and Applications with R. Wiley, 2013.

[7] Dryden, I. L., And Mardia, K. V. Statistical shape analysis: with applications in R. John Wiley \& Sons, 2016.

[8] ERIKSson, J., AND FEnYÖ, D. Improving the success rate of proteome analysis by modeling protein-abundance distributions and experimental designs. Nature Biotechnology (2007).

[9] Goos, P., And Gilmour, G. S. A general strategy for analyzing data from split-plot and multistratum experimental designs. Technometrics (2012).

[10] Harville, D. A. Optimal procedures for some constrained selection problems. Journal of the American Statistical Association 69, 346 (1974), 446-452.

[11] Harville, D. A. Maximum likelihood approaches to variance component estimation and to related problems. Journal of the American statistical association 72, 358 (1977), 320-338. 
[12] Hauser, A., And Buehlmann, P. Characterization and greedy learning of interventional markov equivalence classes of directed acyclic graphs. Journal of Machine Learning Research 13 (2012), 2409-2464.

[13] Henderson, C. R. Best linear unbiased estimation and prediction under a selection model. Biometrics (1975).

[14] Hongzhan, H., Shukla, H. D., Cathy, W., and Satya, S. Challenges and solutions in proteomics. Current genomics (2007).

[15] Johnson, R., And Wichern, D. Applied multivariate statistical analysis. Prentice Hall, 2002.

[16] Kalisch, M., Maechler, M., Colombo, D., Maathuis, M. H., And Buehlmann, P. Causal inference using graphical models with the R package pcalg. Journal of Statistical Software 47, 11 (2012), 1-26.

[17] Kendziorski, C., Irizarry, R. A., Chen, K.-S., HaAg, J. D., And Gould, M. N. On the utility of pooling biological samples in microarray experiments. Proceedings of the National Academy of Sciences (2005).

[18] Klont, F., Bras, L., Wolters, J. C., Ongay, S., Bischoff, R., Halmos, G. B., And Horvatovich, P. Assessment of sample preparation bias in mass spectrometry-based proteomics. Analytical Chemistry (2018).

[19] LAIRD, N., AND WARE, J. Random-effects models for longitudinal data. Biometrics (1982).

[20] LI, W. Volcano plots in analyzing differential expressions with mrna microarrays. Journal of bioinformatics and computational biology (2012).

[21] LovRíc, J. Introducing Proteomics: From concepts to sample separation, mass spectrometry and data analysis. Wiley, 2011.

[22] Morris, J. S., Baggerly, K. A., Gutstein, H. B., And Coombes, K. R. Statistical contributions to proteomic research. Methods in molecular biology (Clifton, N.J.) (2010).

[23] Ness, R. O., Sachs, K., And Vitek, O. From correlation to causality: Statistical approaches to learning regulatory relationships in large-scale biomolecular investigations. Journal of Proteome Research (2016).

[24] O’Rourke, M. B., Town, S., Dalla, P. V., Bicknell, F., Koh Belic, N., Violi, J. P., Steele, J. R., and Padula, M. P. What is normalization? the strategies employed in top-down and bottom-up proteome analysis workflows. Proteomes (2019).

[25] Patterson, H. D., And Thompson, R. Recovery of inter-block information when block sizes are unequal. Biometrika 58, 3 (1971), 545-554. 
[26] Pearl, J. Causality: Models, Reasoning and Inference. Cambridge University Press, 2000.

[27] Pearl, J., And Verma, T. A theory of inferred causation. In Studies in Logic and the Foundations of Mathematics, vol. 134. Elsevier, 1995, pp. 789-811.

[28] Picotti, P., And Aebersold, R. Selected reaction monitoring-based proteomics: workflows, potential, pitfalls and future directions. Nat Methods (2012).

[29] Pinheiro, J., Bates, D., Debroy, S., Sarkar, D., and R Core Team. nlme: Linear and Nonlinear Mixed Effects Models, 2021. R package version 3.1-152.

[30] Pinheiro, J. C., And Bates, D. M. Mixed-Effects Models in $S$ and $S$-PLUS. Springer, 2000.

[31] Searle, S. R., Casella, G., and McCulloch, C. E. Variance Components. Wiley, 1992.

[32] Smilde, A. K., Jansen, J. J., Hoefsloot, H. C. J., Lamers, R.-J. A. N., van Der Greef, J., and Timmerman, M. E. Anova-simultaneous component analysis (asca): a new tool for analyzing designed metabolomics data. Bioinformatics (2005).

[33] Spirtes, P., Glymour, C., And Scheines, R. Causation, Prediction and Search. MIT press, 2000.

[34] Storey, J. D. A direct approach to false discovery rates. Journal of the Royal Statistical Society: Series B (Statistical Methodology) (2002).

[35] Thisted, R. A. Elements of statistical computing. Journal of the American Statistical Association (1988).

[36] Vidova, V., ANd Spacil, Z. A review on mass spectrometry-based quantitative proteomics: Targeted and data independent acquisition. Analytica Chimica Acta 964 (2017).

[37] Wermuth, N., And Cox, D. Graphical models: Overview. In International Encyclopedia of the Social and Behavioral Sciences. Pergamon, 2001.

[38] Wolfinger, R., Gibson, G., Wolfinger, E., Bennett, L., Hamadeh, H., Bushel, P., Afshari, C., And Paules, R. Assessing gene significance from cdna microarray expression data via mixed models. Journal of computational biology : a journal of computational molecular cell biology (2001).

[39] Yu, H., Moharil, J., And Blair, R. H. Bayesnetbp: an r package for probabilistic reasoning in bayesian networks. Journal of Statistical Software (2020). 
[40] Zou, H., Hastie, T., And Tibshirani, R. Sparse principal component analysis. Journal of computational and graphical statistics (2006). 


\section{APÊNDICE A Tabelas suplementares}

\section{A.1 Experimento SRM}

Os resultados apresentados na Seção 5.7 foram baseados nos dados completos, isto é, foram considerados somente os peptídeos presentes em todas as amostras avaliadas. Sendo que, foram avaliados 46 dos 68 peptídeos quantificados pelo experimentos SRM.

Na Tabela A.6 estão apresentados os códigos e a descrição dos 46 peptídeos avaliados as análises multivariadas.

Tabela A.1 - Contrastes na escala logaritmica - Modelo M2 - SRM

\begin{tabular}{lrrrrr}
\hline Contraste & Estimativa & DP & g.l. & $t$ & $p$-valor \\
\hline IGD - ISD & $-0,4369$ & 0,0468 & 9 & $-9,331$ & $<0,0001$ \\
IGD - OFD & $-0,4933$ & 0,0468 & 9 & $-10,535$ & $<0,0001$ \\
IGD - OPD & $-0,4263$ & 0,0468 & 9 & $-9,105$ & $<0,0001$ \\
ISD - OFD & $-0,0564$ & 0,0468 & 9 & $-1,204$ & 0,6396 \\
ISD - OPD & 0,0106 & 0,0468 & 9 & 0,226 & 0,9956 \\
OFD - OPD & 0,0670 & 0,0468 & 9 & 1,430 & 0,5131 \\
\hline
\end{tabular}

Tabela A.2 - Contrastes na escala logaritmica - Modelo M3 - SRM

\begin{tabular}{lrrrrr}
\hline Contraste & Estimativa & DP & g.l. & $t$ & $p$-valor \\
\hline IGD - ISD & $-0,4372$ & 0,0467 & 9 & $-9,369$ & $<0,0001$ \\
IGD - OFD & $-0,4930$ & 0,0467 & 9 & $-10,565$ & $<0,0001$ \\
IGD - OPD & $-0,4259$ & 0,0467 & 9 & $-9,125$ & $<0,0001$ \\
ISD - OFD & $-0,0558$ & 0,0467 & 9 & $-1,196$ & 0,6441 \\
ISD - OPD & 0,0114 & 0,0467 & 9 & 0,244 & 0,9946 \\
OFD - OPD & 0,0672 & 0,0467 & 9 & 1,440 & 0,5080 \\
\hline
\end{tabular}


Figura A.1 - M2: Modelo Linear Misto Homocedástico - SRM
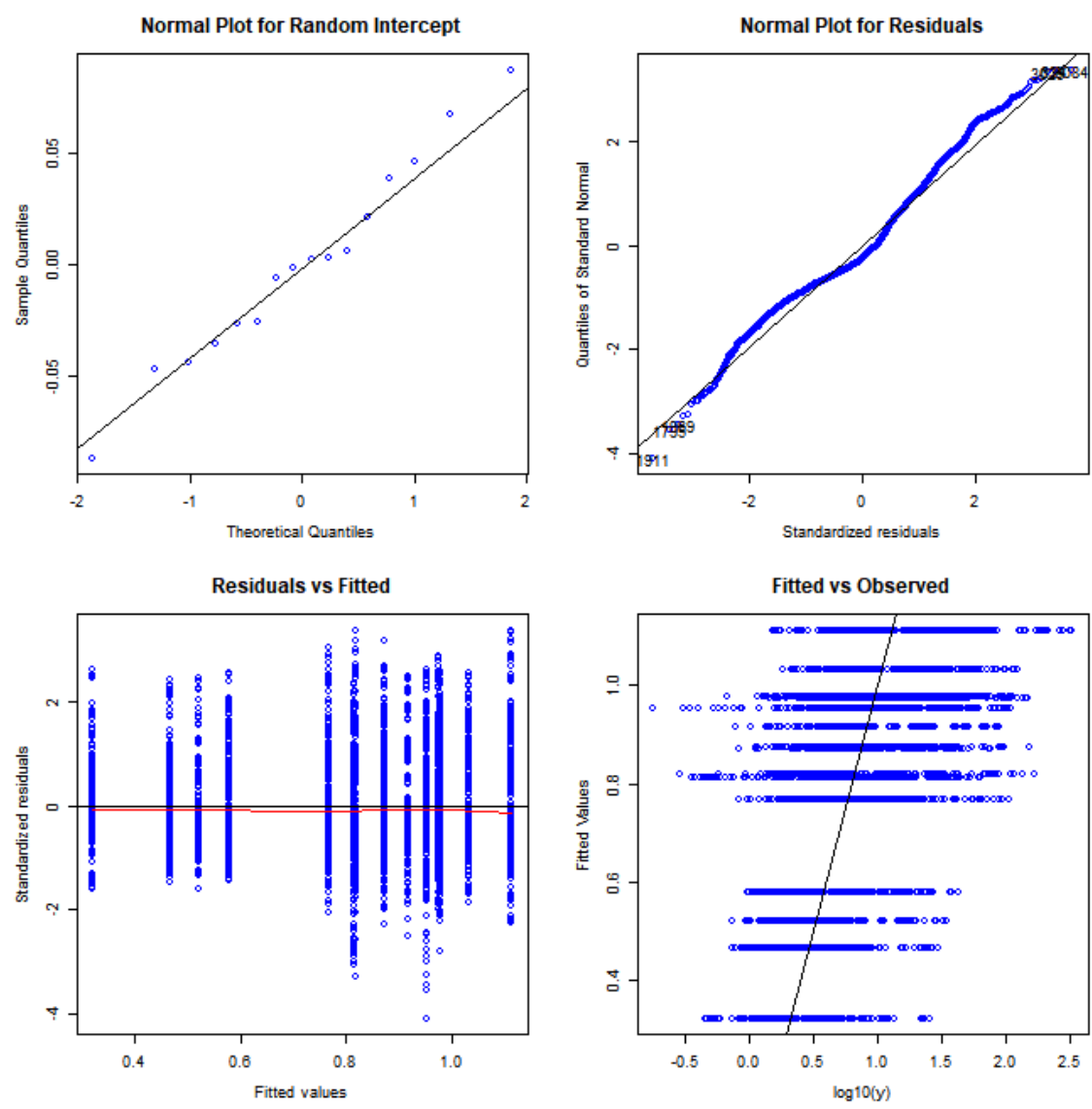
Figura A.2 - M3: Modelo Linear Misto Heterocedástico devido ao efeito de Tecido SRM
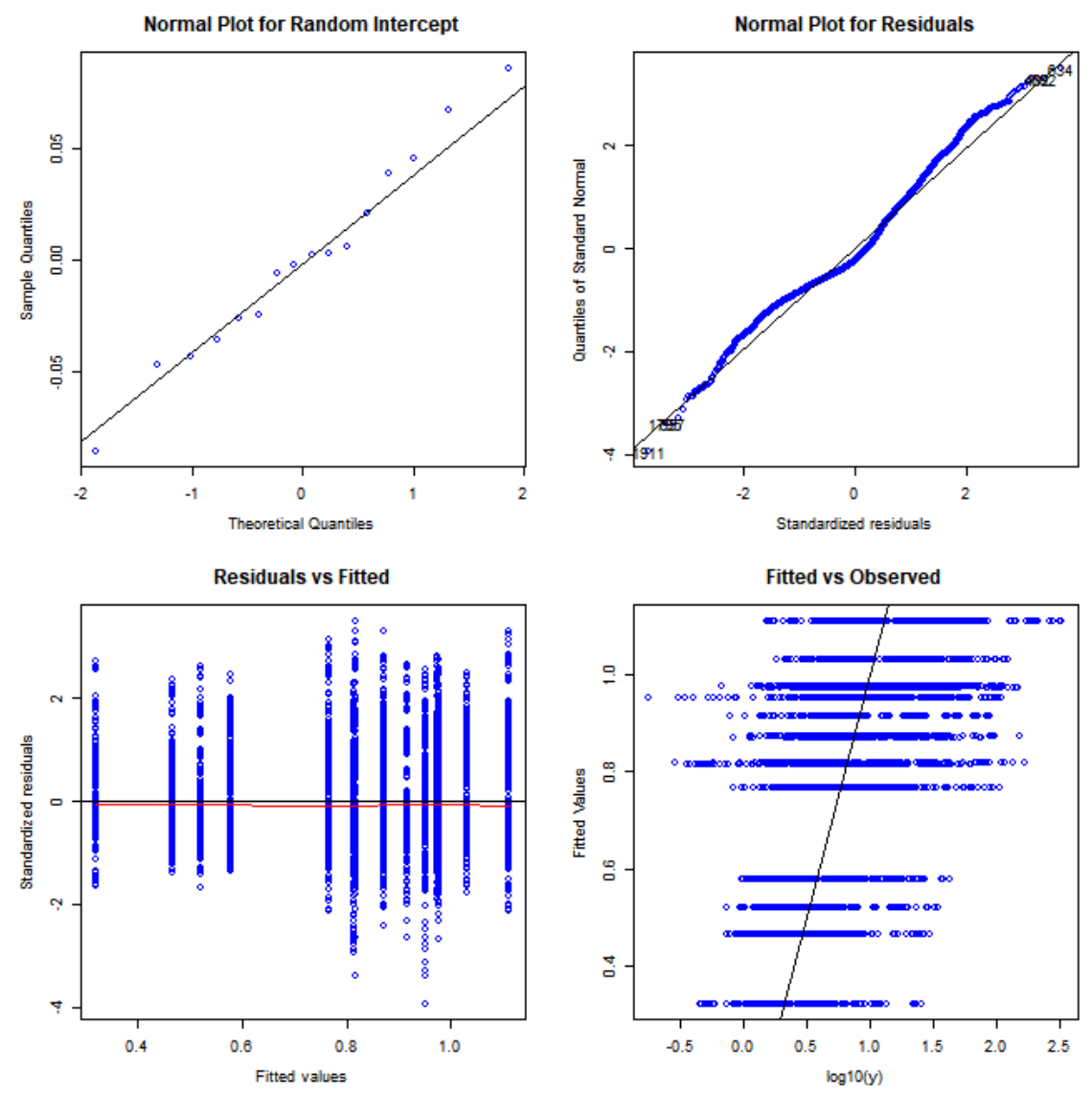
Tabela A.3 - Contrastes na escala logaritmica - Modelo M4 - SRM

\begin{tabular}{lrrrrr}
\hline Contraste & Estimativa & DP & g.l. & $t$ & $p$-valor \\
\hline IGD - ISD & $-0,4386$ & 0,0456 & 9 & $-9,608$ & $<0,0001$ \\
IGD - OFD & $-0,4932$ & 0,0459 & 9 & $-10,738$ & $<0,0001$ \\
IGD - OPD & $-0,4264$ & 0,0455 & 9 & $-9,373$ & $<0,0001$ \\
ISD - OFD & $-0,0547$ & 0,0465 & 9 & $-1,175$ & 0,6560 \\
ISD - OPD & 0,0122 & 0,0461 & 9 & 0,264 & 0,9931 \\
OFD - OPD & 0,0668 & 0,0464 & 9 & 1,441 & 0,5071 \\
\hline
\end{tabular}

Tabela A.4 - M3 versus M2 - SRM

\begin{tabular}{lrrrrrr}
\hline Modelo & g.l. & AIC ML & BIC ML & $\log (L)$ & Razão $L$ & $p$-valor \\
\hline M2 & 7 & 4915,038 & 4960,013 & $-2450,519$ & & \\
M3 & 10 & 4905,378 & 4969,629 & $-2442,689$ & 15,6599 & 0,0013 \\
\hline
\end{tabular}

Tabela A.5 - M4 versus M3 - SRM

\begin{tabular}{lrrrrrr}
\hline Modelo & g.l. & AIC ML & BIC ML & $\log (L)$ & Razão $L$ & $p$-valor \\
\hline M3 & 10 & 4905,378 & 4969,629 & $-2442,689$ & & \\
M4 & 22 & 4810,408 & 4951,760 & $-2383,204$ & 118,9695 & $<0,0001$ \\
\hline
\end{tabular}


Tabela A.6 - Lista de Peptídeos - SRM

\begin{tabular}{|c|c|c|}
\hline Código & Peptídeo & Grafo \\
\hline 1 & IPVGPETLGR & Grafo 2 - SRM \\
\hline 2 & VVDLLAPYAK & Grafo 1 - SRM \\
\hline 3 & IIDAALR & \\
\hline 4 & ADLIAYLK & Grafo 1 - SRM \\
\hline 5 & TGPNLHGLFGR & Grafo 1 - SRM \\
\hline 6 & EFAAGYNVK & Grafo 1 - SRM \\
\hline 7 & TISQHQISTSIITSTQK & Grafo 1 - SRM \\
\hline 8 & FASEIAGVDDLGTTGR & Grafo 1 - SRM \\
\hline 9 & GLLTYTSWEDALSR & Grafo 1 - SRM \\
\hline 10 & DFTPVCTTELGR & Grafo 1 - SRM \\
\hline 11 & GEGGILINSQGER & Grafo 1 - SRM \\
\hline 12 & LGANSLLDLVVFGR & Grafo 1 - SRM \\
\hline 13 & DLVPDLSNFYAQYK & Grafo 1 - SRM \\
\hline 14 & FGFYEVFK & Grafo 2 - SRM \\
\hline 15 & AAYFGIYDTAK & Grafo 1 - SRM \\
\hline 16 & AIWNVINWENVTER & Grafo 2 - SRM \\
\hline 17 & NALANPLYCPDYR & Grafo 2 - SRM \\
\hline 18 & NAVTQEFGPVPDTAR & Grafo 2 - SRM \\
\hline 19 & VAGILTVK & Grafo 2 - SRM \\
\hline 20 & LVAQLYK & Grafo 1 - SRM \\
\hline 21 & ILVPEGTR & Grafo 1 - SRM \\
\hline 22 & VPEANSSWMDTVIR & Grafo 1 - SRM \\
\hline 23 & GLVVPVIR & Grafo 1 - SRM \\
\hline 24 & IYELAAGGTAVGTGLNTR & Grafo 2 - SRM \\
\hline 25 & LNEHFLNTTDFLDTIK & Grafo 1 - SRM \\
\hline 26 & TPYTDVNIVTIR & Grafo 1 - SRM \\
\hline 27 & VAVLGASGGIGQPLSLLLK & Grafo 1 - SRM \\
\hline 28 & VNVPVIGGHAGK & Grafo 1 - SRM \\
\hline 29 & GLSSLLYGSIPK & Grafo 1 - SRM \\
\hline 30 & GTYQGLTATVLK & Grafo 1 - SRM \\
\hline 31 & AVVVNAAQLASYSQSK & Grafo 1 - SRM \\
\hline 32 & GIYTGLSAGLLR & Grafo 1 - SRM \\
\hline 33 & ALIADSGLK & Grafo 1 - SRM \\
\hline 34 & IVFSPEEAK & \\
\hline 35 & LIGPNCPGVINPGECK & Grafo 2 - SRM \\
\hline 36 & IDATQVEVNPFGETPEGQVVCFDAK & Grafo 1 - SRM \\
\hline 37 & DFTATDLSEFAAK & \\
\hline 38 & FFEEVNDPAK & Grafo 1 - SRM \\
\hline 39 & ATAEQISSQTGNK & \\
\hline 40 & VAFITGGGTGLGK & Grafo 1 - SRM \\
\hline 41 & LLYDLADQLHAAVGASR & Grafo 1 - SRM \\
\hline 42 & TIYAGNALCTVK & Grafo 1 - SRM \\
\hline 43 & LGPLQVAR & Grafo 1 - SRM \\
\hline 44 & LSVISVEDPPQR & Grafo 1 - SRM \\
\hline 45 & DGPGFYTTR & \\
\hline 46 & DQLLLGPTYATPK & Grafo 1 - SRM \\
\hline
\end{tabular}




\section{A.2 Experimento DDA - noNorm Peptídeos}

Na Tabela A.13 estão apresentados os códigos e a descrição dos peptídeos apresentados nos grafos probabilísticos apresentados na Seção 5.7, em que contém 54 peptídeos.

Tabela A.7 - Contrastes na escala logarítmica do modelo M2 - DDA noNorm

Peptídeos

\begin{tabular}{lrrrrr}
\hline Contraste & Estimativa & DP & g.l. & $t$ & $p$-valor \\
\hline IGD - ISD & $-0,23119$ & 0,0274 & 9 & $-8,440$ & 0,0001 \\
IGD - OFD & $-0,22331$ & 0,0274 & 9 & $-8,152$ & 0,0001 \\
IGD - OPD & $-0,28645$ & 0,0274 & 9 & $-10,457$ & $<0,0001$ \\
ISD - OFD & 0,00788 & 0,0274 & 9 & 0,288 & 0,9911 \\
ISD - OPD & $-0,05526$ & 0,0274 & 9 & $-2,017$ & 0,2507 \\
OFD - OPD & $-0,06314$ & 0,0274 & 9 & $-2,305$ & 0,1679 \\
\hline
\end{tabular}

Tabela A.8 - Contrastes na escala logarítmica do modelo M3 - DDA noNorm Peptídeos

\begin{tabular}{lrrrrr}
\hline Contraste & Estimativa & DP & g.l. & $t$ & $p$-valor \\
\hline IGD - ISD & $-0,23119$ & 0,0284 & 9 & $-8,150$ & 0,0001 \\
IGD - OFD & $-0,22332$ & 0,0284 & 9 & $-7,872$ & 0,0001 \\
IGD - OPD & $-0,28645$ & 0,0284 & 9 & $-10,097$ & $<0,0001$ \\
ISD - OFD & 0,00788 & 0,0284 & 9 & 0,278 & 0,9920 \\
ISD - OPD & $-0,05526$ & 0,0284 & 9 & $-1,948$ & 0,2752 \\
OFD - OPD & $-0,06314$ & 0,0284 & 9 & $-2,225$ & 0,1880 \\
\hline
\end{tabular}

Tabela A.9 - Contrastes na escala logarítmica do modelo M4 - DDA noNorm Peptídeos

\begin{tabular}{lrrrrr}
\hline Contraste & Estimativa & DP & g.l. & $t$ & $p$-valor \\
\hline IGD - ISD & $-0,23119$ & 0,0281 & 9 & $-8,231$ & 0,0001 \\
IGD - OFD & $-0,22332$ & 0,0281 & 9 & $-7,950$ & 0,0001 \\
IGD - OPD & $-0,28645$ & 0,0281 & 9 & $-10,199$ & $<0,0001$ \\
ISD - OFD & 0,00788 & 0,0281 & 9 & 0,280 & 0,9918 \\
ISD - OPD & $-0,05526$ & 0,0281 & 9 & $-1,967$ & 0,2681 \\
OFD - OPD & $-0,06314$ & 0,0281 & 9 & $-2,247$ & 0,1823 \\
\hline
\end{tabular}


Tabela A.10 - M3 versus M2 - DDA noNorm Peptídeos

\begin{tabular}{lrrrrrr}
\hline Modelo & g.l. & AIC ML & BIC ML & $\log (L)$ & Razão $L$ & $p$-valor \\
\hline M2 & 7 & 3733147 & 3733233 & -1866566 & & \\
M3 & 10 & 3731624 & 3731748 & -1865802 & 1528,454 & $<0,0001$ \\
\hline
\end{tabular}

Tabela A.11 - M4 versus M3 - DDA noNorm Peptídeos

\begin{tabular}{lrrrrrr}
\hline Modelo & g.l. & AIC ML & BIC ML & $\log (L)$ & Razão $L$ & $p$-valor \\
\hline M3 & 10 & 3731624 & 3731748 & -1865802 & & \\
M4 & 22 & 3727615 & 3727887 & -1863785 & 4033,522 & $<0,0001$ \\
\hline
\end{tabular}

Figura A.3 - M2: Modelo Linear Misto Homocedástico - DDA noNorm Peptídeos
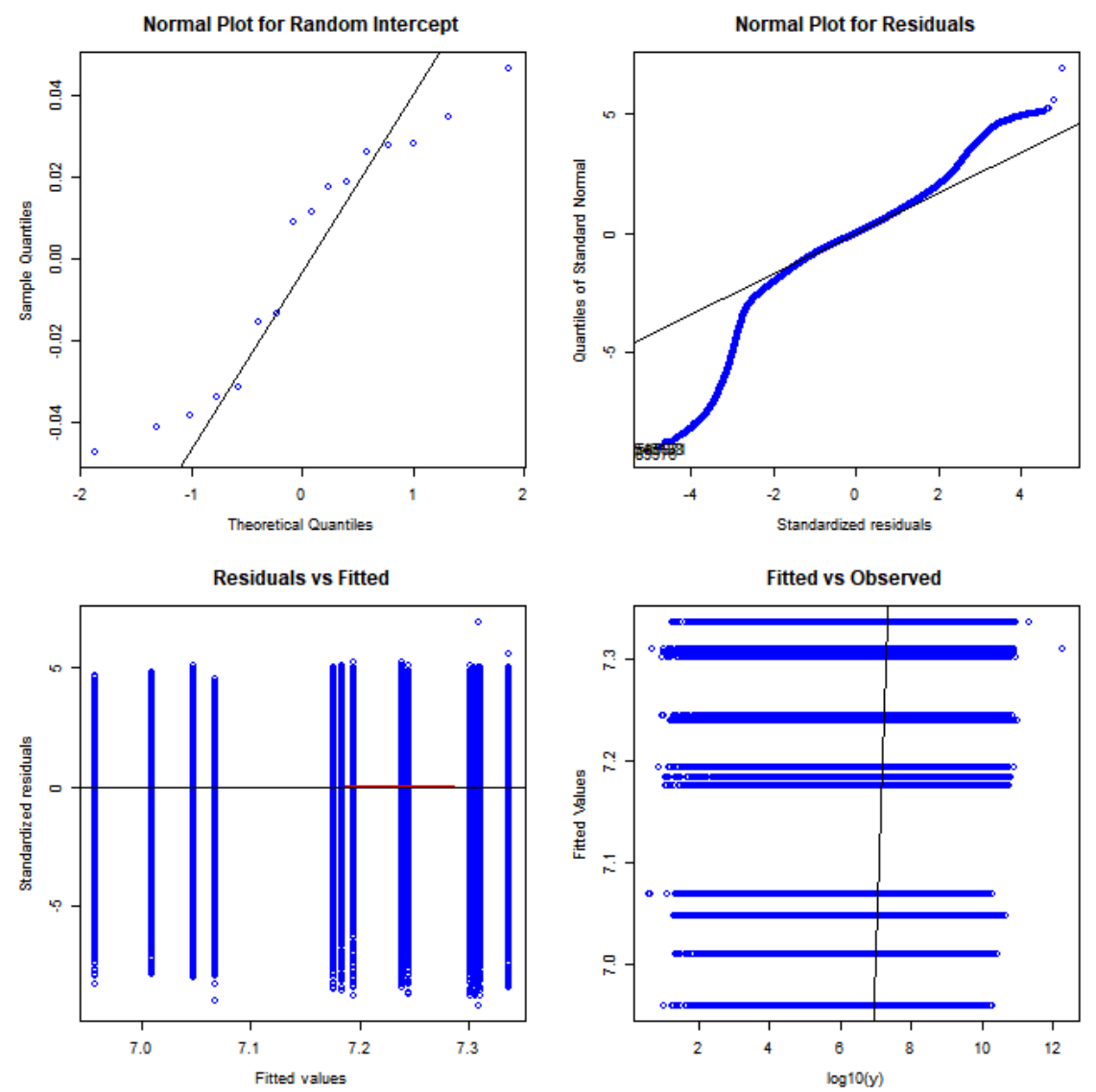
Figura A.4 - M3: Modelo Linear Misto Heterocedástico devido ao efeito de Tecido DDA noNorm Peptídeos
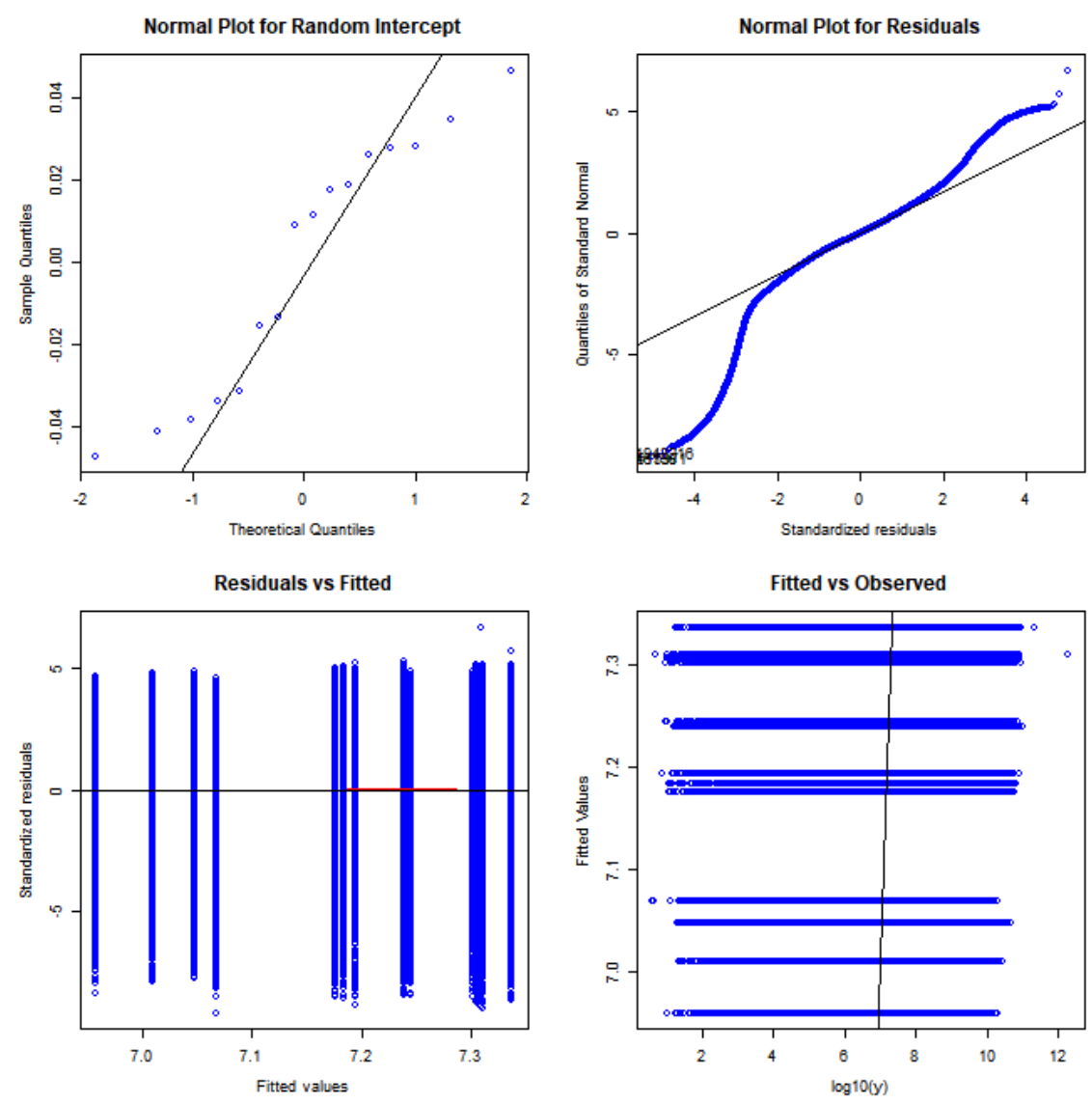
Tabela A.12 - Número de peptídeos significantes por contraste avaliado - DDA noNorm Peptídeos

\begin{tabular}{lr}
\hline Contraste & Número de peptídeos \\
\hline NP: ISD - IGD & 37 \\
NP: OFD - IGD & 30 \\
NP: OFD - ISD & 23 \\
NP: OPD - IGD & 16 \\
NP: OPD - ISD & 14 \\
NP: OPD - OFD & 21 \\
P: ISD - IGD & 19 \\
P: OFD - IGD & 23 \\
P: OFD - ISD & 25 \\
P: OPD - IGD & 9 \\
P: OPD - ISD & 14 \\
P: OPD - OFD & 11 \\
pool: ISD - IGD & 16 \\
pool: OFD - IGD & 11 \\
pool: OFD - ISD & 21 \\
pool: OPD - IGD & 6 \\
pool: OPD - ISD & 15 \\
pool: OPD - OFD & 12 \\
T: ISD - IGD & 16 \\
T: OFD - IGD & 19 \\
T: OFD - ISD & 19 \\
T: OPD - IGD & 4 \\
T: OPD - ISD & 12 \\
T: OPD - OFD & 16 \\
\hline
\end{tabular}


Tabela A.13 - Lista de Peptídeos - DDA noNorm

\begin{tabular}{|c|c|c|}
\hline Código & Peptídeo & Grafo \\
\hline 356 & SLLQALNEVK & Grafo 1 - DDA noNorm Peptídeos \\
\hline 799 & VVYLR & Grafo 1 - DDA noNorm Peptídeos \\
\hline 1105 & DAIAQAVR & Grafo 1 - DDA noNorm Peptídeos \\
\hline 1142 & NNASTDYDLSDK & Grafo 1 - DDA noNorm Peptídeos \\
\hline 1782 & GLVYETSVLDPDEGIR & Grafo 1 - DDA noNorm Peptídeos \\
\hline 1817 & YTIENPR & Grafo 1 - DDA noNorm Peptídeos \\
\hline 2117 & GEDEEENNLEVR & Grafo 1 - DDA noNorm Peptídeos \\
\hline 2765 & DPAEGDGAQPEETPR & Grafo 1 - DDA noNorm Peptídeos \\
\hline 2956 & AYEKPPEK & Grafo 1 - DDA noNorm Peptídeos \\
\hline 3219 & AGDFLEANYMNLQR & Grafo 1 - DDA noNorm Peptídeos \\
\hline 3655 & RPDQQLQGEGK & Grafo 1 - DDA noNorm Peptídeos \\
\hline 3659 & IIDVVYNASNNELVR & Grafo 1 - DDA noNorm Peptídeos \\
\hline 3715 & DLTGVQNLR & Grafo 1 - DDA noNorm Peptídeos \\
\hline 4080 & VGEQAQVVIIDMNDPSNPIR & Grafo 1 - DDA noNorm Peptídeos \\
\hline 4187 & HSQDLAFLSMLNDIAAVPATAMPFR & Grafo 1 - DDA noNorm Peptídeos \\
\hline 4402 & LLDAVDTYIPVPAR & Grafo 1 - DDA noNorm Peptídeos \\
\hline 4696 & IEPGVDPDDTYNETPYEK & Grafo 1 - DDA noNorm Peptídeos \\
\hline 133 & LLLQVQHASK & Grafo 2 - DDA noNorm Peptídeos \\
\hline 144 & KFDQLLAEEK & Grafo 2 - DDA noNorm Peptídeos \\
\hline 160 & AHSSMVGVNLPQK & Grafo 2 - DDA noNorm Peptídeos \\
\hline 286 & LFKPGQEAVK & Grafo 2 - DDA noNorm Peptídeos \\
\hline 421 & AVTEQGHELSNEER & Grafo 2 - DDA noNorm Peptídeos \\
\hline 435 & KLNVTEQEK & Grafo 2 - DDA noNorm Peptídeos \\
\hline 607 & HLIPAANTGESK & Grafo 2 - DDA noNorm Peptídeos \\
\hline 682 & IFAPNHVVAK & Grafo 2 - DDA noNorm Peptídeos \\
\hline 737 & GHLENNPALEK & Grafo 2 - DDA noNorm Peptídeos \\
\hline 1715 & AWDDFFPGSDR & Grafo 2 - DDA noNorm Peptídeos \\
\hline 2034 & STTTGHLIYK & Grafo 2 - DDA noNorm Peptídeos \\
\hline 2862 & NLQPASEYTVSLVAIK & Grafo 2 - DDA noNorm Peptídeos \\
\hline 3722 & FGYVDFESAEDLEK & Grafo 2 - DDA noNorm Peptídeos \\
\hline 657 & SSLVLR & Grafo 3 - DDA noNorm Peptideos \\
\hline 1106 & ESSSHHPGIAEFPSR & Grafo 3 - DDA noNorm Peptideos \\
\hline 1113 & EGIVALR & Grafo 3 - DDA noNorm Peptideos \\
\hline 1257 & EPPLELK & Grafo 3 - DDA noNorm Peptideos \\
\hline 1294 & AQEGLRPGTLC $(+57.02)$ TVAGWGR & Grafo 3 - DDA noNorm Peptideos \\
\hline 1574 & ASIHEAWTDGK & Grafo 3 - DDA noNorm Peptideos \\
\hline 2236 & LLLIQAFRPDR & Grafo 3 - DDA noNorm Peptideos \\
\hline 2441 & GDVDISLPK & Grafo 3 - DDA noNorm Peptideos \\
\hline 2897 & HITIFSPEGR & Grafo 3 - DDA noNorm Peptideos \\
\hline 3181 & LC(+7.02)DNLSTK & Grafo 3 - DDA noNorm Peptideos \\
\hline 3716 & LNVTEQEK & Grafo 3 - DDA noNorm Peptideos \\
\hline 3741 & TILMMGR & Grafo 3 - DDA noNorm Peptideos \\
\hline 773 & VPGGLAEHK & Grafo 4 - DDA noNorm Peptideos \\
\hline 776 & EQEITAVQAR & Grafo 4 - DDA noNorm Peptideos \\
\hline 840 & VHSPSGALEEC $(+57.02)$ YVTEIDQDK & Grafo 4 - DDA noNorm Peptideos \\
\hline 1004 & VVNVSSIMSVR & Grafo 4 - DDA noNorm Peptideos \\
\hline 1087 & SVPTWLK & Grafo 4 - DDA noNorm Peptideos \\
\hline 1176 & LGEQNFHK & Grafo 4 - DDA noNorm Peptideos \\
\hline 1245 & SETITEEELVGLMNK & Grafo 4 - DDA noNorm Peptideos \\
\hline 2775 & VGLEHLR & Grafo 4 - DDA noNorm Peptideos \\
\hline 2985 & DQNVFVAQK & Grafo 4 - DDA noNorm Peptideos \\
\hline 3369 & GGVADALLYR & Grafo 4 - DDA noNorm Peptideos \\
\hline 3464 & FATTFYQHLADSK & Grafo 4 - DDA noNorm Peptideos \\
\hline 4423 & VAVVTYNNEVTTEIR & Grafo 4 - DDA noNorm Peptideos \\
\hline
\end{tabular}




\section{A.3 Experimento DDA - TIC Peptídeos}

Na Tabela A.15 estão apresentados os códigos e a descrição dos peptídeos apresentados nos grafos probabilísticos apresentados na Seção 5.7, contendo 33 peptídeos.

Tabela A.14 - Número de peptídeos significantes por contraste avaliado - DDA TIC Peptídeos

\begin{tabular}{lr}
\hline Contraste & Número de peptídeos \\
\hline NP: ISD - IGD & 38 \\
NP: OFD - IGD & 28 \\
NP: OFD - ISD & 23 \\
NP: OPD - IGD & 17 \\
NP: OPD - ISD & 15 \\
NP: OPD - OFD & 21 \\
P: ISD - IGD & 25 \\
P: OFD - IGD & 24 \\
P: OFD - ISD & 26 \\
P: OPD - IGD & 10 \\
P: OPD - ISD & 16 \\
P: OPD - OFD & 11 \\
pool: ISD - IGD & 24 \\
pool: OFD - IGD & 14 \\
pool: OFD - ISD & 22 \\
pool: OPD - IGD & 6 \\
pool: OPD - ISD & 14 \\
pool: OPD - OFD & 14 \\
T: ISD - IGD & 19 \\
T: OFD - IGD & 23 \\
T: OFD - ISD & 21 \\
T: OPD - IGD & 6 \\
T: OPD - ISD & 11 \\
T: OPD - OFD & 14 \\
\hline
\end{tabular}


Tabela A.15 - Lista de Peptídeos - DDA TIC

\begin{tabular}{rll}
\hline Código & Peptídeo & Grafo \\
\hline 976 & ANLQIDQINTDLNLER & Grafo 1 - DDA TIC Peptídeos \\
1265 & GIQEEMEALVK & Grafo 1 - DDA TIC Peptídeos \\
2018 & GPSGLLLYNGQK & Grafo 1 - DDA TIC Peptídeos \\
2704 & MIPC(+57.02)DFLIPVQTQHPIR & Grafo 1 - DDA TIC Peptídeos \\
2850 & YLALESMC(+57.02)TLASSEFSHEAVK & Grafo 1 - DDA TIC Peptídeos \\
2953 & GEGPEVDVNLPK & Grafo 1 - DDA TIC Peptídeos \\
3358 & DDLYVSDAFHK & Grafo 1 - DDA TIC Peptídeos \\
3576 & YQEEFEHFQQELDK & Grafo 1 - DDA TIC Peptídeos \\
3997 & EFTRPEEIFLR & Grafo 1 - DDA TIC Peptídeos \\
4083 & IDSEGGVSANHTSR & Grafo 1 - DDA TIC Peptídeos \\
4094 & EELGLIEQAYDNPHEALSR & Grafo 1 - DDA TIC Peptídeos \\
4587 & LVVDLTDIDPDVAYSSVPYEK & Grafo 1 - DDA TIC Peptídeos \\
84 & VVDLLAPYAK & Grafo 2 - DDA TIC Peptídeos \\
147 & DSGFQMNQLR & Grafo 2 - DDA TIC Peptídeos \\
618 & GDLGIEIPAEK & Grafo 2 - DDA TIC Peptídeos \\
1447 & DNLAEDIMR & Grafo 2 - DDA TIC Peptídeos \\
2626 & EYHPDKNPNAGDK & Grafo 2 - DDA TIC Peptídeos \\
3378 & KDSGFQMNQLR & Grafo 2 - DDA TIC Peptídeos \\
3671 & SLDMDSIIAEVK & Grafo 2 - DDA TIC Peptídeos \\
4144 & ASEEHLK & Grafo 2 - DDA TIC Peptídeos \\
4249 & VINQILTEMDGMSTK & Grafo 2 - DDA TIC Peptídeos \\
4385 & ILLAELEQLK & Grafo 2 - DDA TIC Peptídeos \\
4540 & ESTGAQVQVAGDMLPNSTER & Grafo 2 - DDA TIC Peptídeos \\
281 & WQEEMELYR & Grafo 3 - DDA TIC Peptídeos \\
571 & DFPIAK & Grafo 3 - DDA TIC Peptídeos \\
940 & SNYFLK & Grafo 3 - DDA TIC Peptídeos \\
2015 & TYALNAAGVSK & Grafo 3 - DDA TIC Peptídeos \\
2348 & ETDSSSASAATPSK & Grafo 3 - DDA TIC Peptídeos \\
2511 & QMVIDVLHPGK & Grafo 3 - DDA TIC Peptídeos \\
2771 & GQSSWGTGESFR & Grafo 3 - DDA TIC Peptídeos \\
3139 & FSGVPDR & Grafo 3 - DDA TIC Peptídeos \\
3587 & IWDVVEK & Grafo 3 - DDA TIC Peptídeos \\
4152 & LHVDPENFR & Grafo 3 - DDA TIC Peptídeos \\
\hline & &
\end{tabular}




\section{A.4 Experimento DDA noNorm Proteínas}

Na Tabela A.22 estão apresentados os códigos das proteínas apresentados nos grafos probabilísticos na Seção 5.7, contendo 70 proteínas.

Tabela A.16 - Contrastes na escala logarítmica M2 - DDA noNorm Proteínas

\begin{tabular}{lrrrrr}
\hline Contraste & Estimativa & DP & g.l. & $t$ & $p$-valor \\
\hline IGD - ISD & $-0,3225$ & 0,0313 & 9 & $-10,294$ & $<0,0001$ \\
IGD - OFD & $-0,3407$ & 0,0313 & 9 & $-10,874$ & $<0,0001$ \\
IGD - OPD & $-0,3743$ & 0,0313 & 9 & $-11,949$ & $<0,0001$ \\
ISD - OFD & $-0,0182$ & 0,0313 & 9 & $-0,580$ & 0,9356 \\
ISD - OPD & $-0,0518$ & 0,0313 & 9 & $-1,654$ & 0,3987 \\
OFD - OPD & $-0,0336$ & 0,0313 & 9 & $-1,074$ & 0,7129 \\
\hline
\end{tabular}

Tabela A.17 - Contrastes na escala logarítmica M3 - DDA noNorm Proteínas

\begin{tabular}{lrrrrr}
\hline Contraste & Estimativa & DP & g.l. & $t$ & $p$-valor \\
\hline IGD - ISD & $-0,3225$ & 0,0303 & 9 & $-10,636$ & $<0,0001$ \\
IGD - OFD & $-0,3407$ & 0,0303 & 9 & $-11,233$ & $<0,0001$ \\
IGD - OPD & $-0,3743$ & 0,0303 & 9 & $-12,343$ & $<0,0001$ \\
ISD - OFD & $-0,0181$ & 0,0303 & 9 & $-0,599$ & 0,9300 \\
ISD - OPD & $-0,0518$ & 0,0303 & 9 & $-1,707$ & 0,3741 \\
OFD - OPD & $-0,0336$ & 0,0303 & 9 & $-1,108$ & 0,6937 \\
\hline
\end{tabular}

Tabela A.18 - Contrastes na escala logarítmica M4 - DDA noNorm Proteínas

\begin{tabular}{lrrrrr}
\hline Contraste & Estimativa & DP & g.l. & $t$ & $p$-valor \\
\hline IGD - ISD & $-0,3225$ & 0,0322 & 9 & $-10,016$ & $<0,0001$ \\
IGD - OFD & $-0,3407$ & 0,0322 & 9 & $-10,576$ & $<0,0001$ \\
IGD - OPD & $-0,3743$ & 0,0322 & 9 & $-11,621$ & $<0,0001$ \\
ISD - OFD & $-0,0182$ & 0,0322 & 9 & $-0,564$ & 0,9403 \\
ISD - OPD & $-0,0518$ & 0,0322 & 9 & $-1,608$ & 0,4209 \\
OFD - OPD & $-0,0336$ & 0,0322 & 9 & $-1,044$ & 0,7296 \\
\hline
\end{tabular}


Tabela A.19 - M3 versus M2 - DDA noNorm Proteínas

\begin{tabular}{lrrrrrr}
\hline Modelo & g.l. & AIC ML & BIC ML & $\log (L)$ & Razão $L$ & $p$-valor \\
\hline M2 & 7 & 3733147 & 3733233 & -1866566 & & \\
M3 & 10 & 3731624 & 3731748 & -1865802 & 1528,454 & $<0,0001$ \\
\hline
\end{tabular}

Tabela A.20 - M4 versus M3 - DDA noNorm Proteínas

\begin{tabular}{lrrrrrr}
\hline Modelo & g.l. & AIC ML & BIC ML & $\log (L)$ & Razão $L$ & $p$-valor \\
\hline M3 & 10 & 3731624 & 3731748 & -1865802 & & \\
M4 & 22 & 3727615 & 3727887 & -1863785 & 4033,522 & $<0,0001$ \\
\hline
\end{tabular}

Figura A.5 - M2: Modelo Linear Misto Homocedástico - DDA noNorm Proteínas
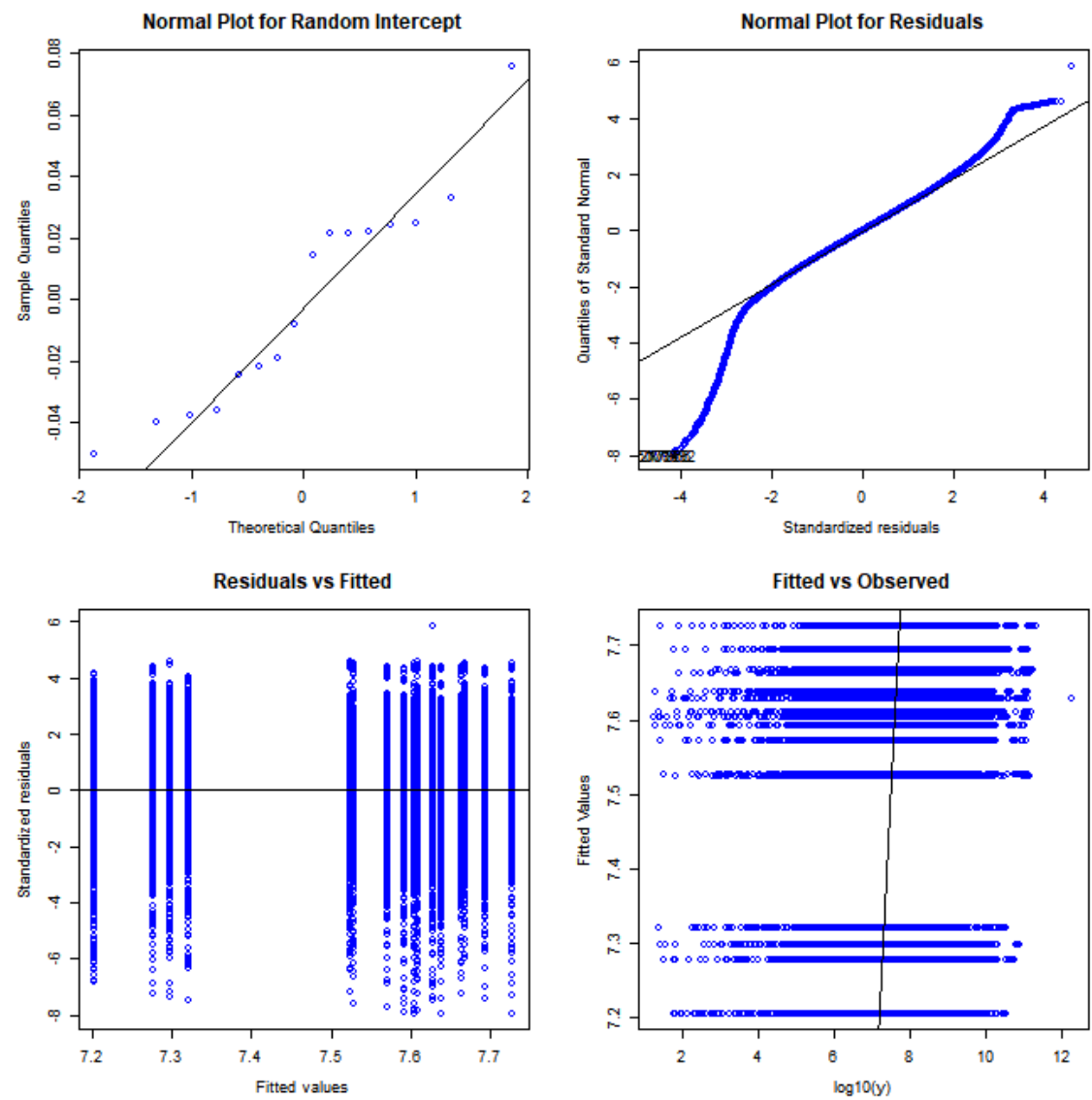
Figura A.6 - M3: Modelo Linear Misto Heterocedástico devido ao efeito de Tecido DDA noNorm Proteínas
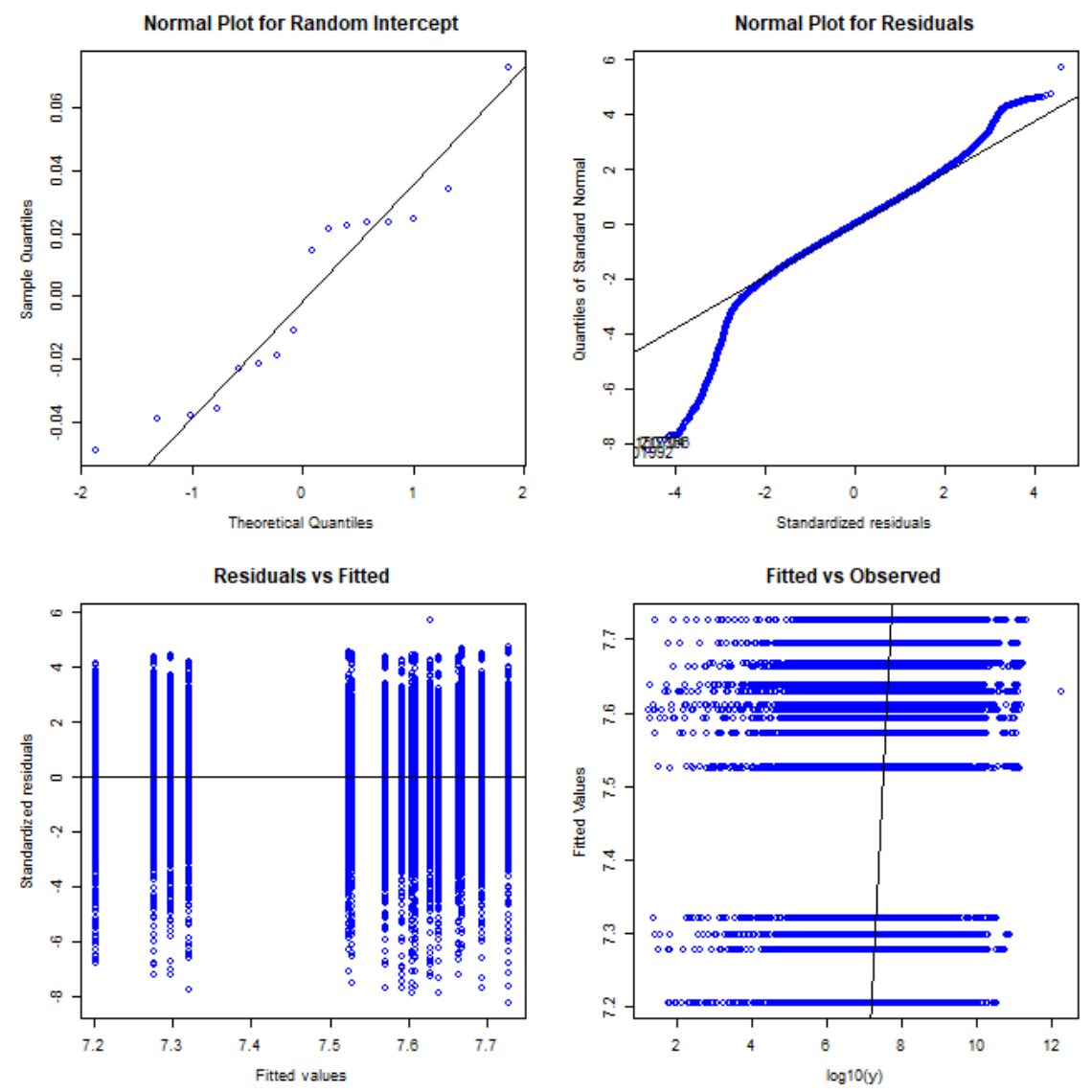
Tabela A.21 - Número de proteínas significantes por contraste - DDA noNorm Proteínas

\begin{tabular}{lr}
\hline Contraste & Número de proteínas \\
\hline NP: ISD - IGD & 7 \\
NP: OFD - IGD & 8 \\
NP: OFD - ISD & 11 \\
NP: OPD - IGD & 5 \\
NP: OPD - ISD & 2 \\
NP: OPD - OFD & 5 \\
P: ISD - IGD & 7 \\
P: OFD - IGD & 9 \\
P: OFD - ISD & 9 \\
P: OPD - IGD & 6 \\
P: OPD - ISD & 5 \\
P: OPD - OFD & 7 \\
pool: ISD - IGD & 10 \\
pool: OFD - IGD & 7 \\
pool: OFD - ISD & 8 \\
pool: OPD - IGD & 1 \\
pool: OPD - ISD & 2 \\
pool: OPD - OFD & 6 \\
T: ISD - IGD & 6 \\
T: OFD - IGD & 4 \\
T: OFD - ISD & 8 \\
T: OPD - IGD & 2 \\
T: OPD - ISD & 3 \\
T: OPD - OFD & 5 \\
\hline
\end{tabular}


Tabela A.22 - Lista de Proteínas - DDA noNorm

\begin{aligned} & \hline Proteína Grafo \\ & \hline 181 Grafo 1 - DDA noNorm Proteinas \\ & 645 Grafo 1 - DDA noNorm Proteinas \\ & 223 Grafo 1 - DDA noNorm Proteinas \\ & 502 Grafo 1 - DDA noNorm Proteinas \\ & 1016 Grafo 1 - DDA noNorm Proteinas \\ & 1275 Grafo 1 - DDA noNorm Proteinas \\ & 2257 Grafo 1 - DDA noNorm Proteinas \\ & 53 Grafo 1 - DDA noNorm Proteinas \\ & 1589 Grafo 1 - DDA noNorm Proteinas \\ & 2017 Grafo 1 - DDA noNorm Proteinas \\ & 535 Grafo 1 - DDA noNorm Proteinas \\ & 1886 Grafo 1 - DDA noNorm Proteinas \\ & 154 Grafo 1 - DDA noNorm Proteinas \\ & 1427 Grafo 1 - DDA noNorm Proteinas \\ & 1234 Grafo 1 - DDA noNorm Proteinas \\ & 63 Grafo 1 - DDA noNorm Proteinas \\ & 866 Grafo 1 - DDA noNorm Proteinas \\ & 594 Grafo 1 - DDA noNorm Proteinas \\ & 1493 Grafo 1 - DDA noNorm Proteinas \\ & 98 Grafo 1 - DDA noNorm Proteinas \\ & 232 Grafo 1 - DDA noNorm Proteinas \\ & 1790 Grafo 1 - DDA noNorm Proteinas \\ & 2280 Grafo 1 - DDA noNorm Proteinas \\ & 2423 Grafo 1 - DDA noNorm Proteinas \\ & 261 Grafo 1 - DDA noNorm Proteinas \\ & 1997 Grafo 1 - DDA noNorm Proteinas \\ & 1688 Grafo 1 - DDA noNorm Proteinas \\ & 1285 Grafo 1 - DDA noNorm Proteinas \\ & 2772 Grafo 1 - DDA noNorm Proteinas \\ & 2512 Grafo 2 - DDA noNorm Proteinas \\ & 782 Grafo 2 - DDA noNorm Proteinas \\ & 258 Grafo 2 - DDA noNorm Proteinas \\ & \hline Grafo 2 - DDA noNorm Proteinas \\ & \hline Grafo 2 - DDA noNorm Proteinas \\ & \hline\end{aligned}

\begin{aligned} & \hline Proteína Grafo \\ & \hline 521 Grafo 2 - DDA noNorm Proteinas \\ & 42 Grafo 2 - DDA noNorm Proteinas \\ & 421 Grafo 2 - DDA noNorm Proteinas \\ & 1104 Grafo 2 - DDA noNorm Proteinas \\ & 249 Grafo 2 - DDA noNorm Proteinas \\ & 263 Grafo 2 - DDA noNorm Proteinas \\ & 326 Grafo 2 - DDA noNorm Proteinas \\ & 689 Grafo 2 - DDA noNorm Proteinas \\ & 921 Grafo 2 - DDA noNorm Proteinas \\ & 1724 Grafo 2 - DDA noNorm Proteinas \\ & 1053 Grafo 2 - DDA noNorm Proteinas \\ & 1541 Grafo 2 - DDA noNorm Proteinas \\ & 1669 Grafo 2 - DDA noNorm Proteinas \\ & 137 Grafo 2 - DDA noNorm Proteinas \\ & 85 Grafo 2 - DDA noNorm Proteinas \\ & 1536 Grafo 2 - DDA noNorm Proteinas \\ & 859 Grafo 3 - DDA noNorm Proteinas \\ & 840 Grafo 3 - DDA noNorm Proteinas \\ & 2792 Grafo 3 - DDA noNorm Proteinas \\ & 2518 Grafo 3 - DDA noNorm Proteinas \\ & 1620 Grafo 3 - DDA noNorm Proteinas \\ & 641 Grafo 3 - DDA noNorm Proteinas \\ & 711 Grafo 3 - DDA noNorm Proteinas \\ & 1357 Grafo 3 - DDA noNorm Proteinas \\ & 1326 Grafo 3 - DDA noNorm Proteinas \\ & 1623 Grafo 3 - DDA noNorm Proteinas \\ & 2687 Grafo 3 - DDA noNorm Proteinas \\ & 2428 Grafo 3 - DDA noNorm Proteinas \\ & 1632 Grafo 3 - DDA noNorm Proteinas \\ & 944 Grafo 3 - DDA noNorm Proteinas \\ & 1539 Grafo 3 - DDA noNorm Proteinas \\ & 1156 Grafo 3 - DDA noNorm Proteinas \\ & \hline Grafo 3 - DDA noNorm Proteinas \\ & \hline Grafo 3 - DDA noNorm Proteinas \\ & \hline\end{aligned}




\section{A.5 Experimento DDA - TIC Proteínas}

Na Tabela A.24 estão apresentados os códigos das proteínas apresentados nos grafos probabilísticos na Seção 5.7 , contendo 88 proteínas.

Tabela A.23 - Número de proteínas significantes por contraste - DDA TIC Proteínas

\begin{tabular}{lr}
\hline Contraste & Número de proteínas \\
\hline NP: ISD - IGD & 7 \\
NP: OFD - IGD & 7 \\
NP: OFD - ISD & 10 \\
NP: OPD - IGD & 5 \\
NP: OPD - ISD & 2 \\
NP: OPD - OFD & 8 \\
P: ISD - IGD & 6 \\
P: OFD - IGD & 8 \\
P: OFD - ISD & 9 \\
P: OPD - IGD & 6 \\
P: OPD - ISD & 5 \\
P: OPD - OFD & 8 \\
pool: ISD - IGD & 4 \\
pool: OFD - IGD & 6 \\
pool: OFD - ISD & 10 \\
pool: OPD - IGD & 1 \\
pool: OPD - ISD & 2 \\
pool: OPD - OFD & 6 \\
T: ISD - IGD & 3 \\
T: OFD - IGD & 2 \\
T: OFD - ISD & 8 \\
T: OPD - IGD & 2 \\
T: OPD - ISD & 3 \\
T: OPD - OFD & 5 \\
\hline
\end{tabular}


Tabela A.24 - Lista de Proteínas - DDA TIC

\begin{tabular}{|c|c|}
\hline Proteína & Grafo \\
\hline 142 & Grafo 1 - DDA TIC Proteinas \\
\hline 332 & Grafo 1 - DDA TIC Proteinas \\
\hline 228 & Grafo 1 - DDA TIC Proteinas \\
\hline 629 & Grafo 1 - DDA TIC Proteinas \\
\hline 1466 & Grafo 1 - DDA TIC Proteinas \\
\hline 58 & Grafo 1 - DDA TIC Proteinas \\
\hline 1236 & Grafo 1 - DDA TIC Proteinas \\
\hline 295 & Grafo 1 - DDA TIC Proteinas \\
\hline 531 & Grafo 1 - DDA TIC Proteinas \\
\hline 1162 & Grafo 1 - DDA TIC Proteinas \\
\hline 901 & Grafo 1 - DDA TIC Proteinas \\
\hline 374 & Grafo 1 - DDA TIC Proteinas \\
\hline 2105 & Grafo 1 - DDA TIC Proteinas \\
\hline 2695 & Grafo 1 - DDA TIC Proteinas \\
\hline 884 & Grafo 1 - DDA TIC Proteinas \\
\hline 2847 & Grafo 1 - DDA TIC Proteinas \\
\hline 1053 & Grafo 1 - DDA TIC Proteinas \\
\hline 2740 & Grafo 1 - DDA TIC Proteinas \\
\hline 1942 & Grafo 1 - DDA TIC Proteinas \\
\hline 879 & Grafo 1 - DDA TIC Proteinas \\
\hline 2733 & Grafo 1 - DDA TIC Proteinas \\
\hline 2816 & Grafo 1 - DDA TIC Proteinas \\
\hline 2465 & Grafo 1 - DDA TIC Proteinas \\
\hline 3 & Grafo 1 - DDA TIC Proteinas \\
\hline 113 & Grafo 1 - DDA TIC Proteinas \\
\hline 2130 & Grafo 1 - DDA TIC Proteinas \\
\hline 1414 & Grafo 1 - DDA TIC Proteinas \\
\hline 1245 & Grafo 1 - DDA TIC Proteinas \\
\hline 1194 & Grafo 1 - DDA TIC Proteinas \\
\hline 1765 & Grafo 1 - DDA TIC Proteinas \\
\hline 991 & Grafo 1 - DDA TIC Proteinas \\
\hline 40 & Grafo 2 - DDA TIC Proteinas \\
\hline 125 & Grafo 2 - DDA TIC Proteinas \\
\hline 48 & Grafo 2 - DDA TIC Proteinas \\
\hline 1234 & Grafo 2 - DDA TIC Proteinas \\
\hline 732 & Grafo 2 - DDA TIC Proteinas \\
\hline 52 & Grafo 2 - DDA TIC Proteinas \\
\hline 272 & Grafo 2 - DDA TIC Proteinas \\
\hline 1480 & Grafo 2 - DDA TIC Proteinas \\
\hline 422 & Grafo 2 - DDA TIC Proteinas \\
\hline 445 & Grafo 2 - DDA TIC Proteinas \\
\hline 869 & Grafo 2 - DDA TIC Proteinas \\
\hline 830 & Grafo 2 - DDA TIC Proteinas \\
\hline 1381 & Grafo 2 - DDA TIC Proteinas \\
\hline
\end{tabular}

\begin{tabular}{|c|c|}
\hline Proteína & Grafo \\
\hline 1711 & Grafo 2 - DDA TIC Proteinas \\
\hline 239 & Grafo 2 - DDA TIC Proteinas \\
\hline 739 & Grafo 2 - DDA TIC Proteinas \\
\hline 1666 & Grafo 2 - DDA TIC Proteinas \\
\hline 555 & Grafo 2 - DDA TIC Proteinas \\
\hline 526 & Grafo 2 - DDA TIC Proteinas \\
\hline 2487 & Grafo 2 - DDA TIC Proteinas \\
\hline 1158 & Grafo 2 - DDA TIC Proteinas \\
\hline 1326 & Grafo 2 - DDA TIC Proteinas \\
\hline 3166 & Grafo 2 - DDA TIC Proteinas \\
\hline 3086 & Grafo 2 - DDA TIC Proteinas \\
\hline 1847 & Grafo 2 - DDA TIC Proteinas \\
\hline 209 & Grafo 2 - DDA TIC Proteinas \\
\hline 2914 & Grafo 2 - DDA TIC Proteinas \\
\hline 1965 & Grafo 2 - DDA TIC Proteinas \\
\hline 924 & Grafo 2 - DDA TIC Proteinas \\
\hline 899 & Grafo 2 - DDA TIC Proteinas \\
\hline 1490 & Grafo 2 - DDA TIC Proteinas \\
\hline 5 & Grafo 3 - DDA TIC Proteinas \\
\hline 233 & Grafo 3 - DDA TIC Proteinas \\
\hline 439 & Grafo 3 - DDA TIC Proteinas \\
\hline 1331 & Grafo 3 - DDA TIC Proteinas \\
\hline 831 & Grafo 3 - DDA TIC Proteinas \\
\hline 495 & Grafo 3 - DDA TIC Proteinas \\
\hline 689 & Grafo 3 - DDA TIC Proteinas \\
\hline 292 & Grafo 3 - DDA TIC Proteinas \\
\hline 591 & Grafo 3 - DDA TIC Proteinas \\
\hline 1961 & Grafo 3 - DDA TIC Proteinas \\
\hline 900 & Grafo 3 - DDA TIC Proteinas \\
\hline 776 & Grafo 3 - DDA TIC Proteinas \\
\hline 1172 & Grafo 3 - DDA TIC Proteinas \\
\hline 1867 & Grafo 3 - DDA TIC Proteinas \\
\hline 822 & Grafo 3 - DDA TIC Proteinas \\
\hline 803 & Grafo 3 - DDA TIC Proteinas \\
\hline 1511 & Grafo 3 - DDA TIC Proteinas \\
\hline 1974 & Grafo 3 - DDA TIC Proteinas \\
\hline 778 & Grafo 3 - DDA TIC Proteinas \\
\hline 1468 & Grafo 3 - DDA TIC Proteinas \\
\hline 671 & Grafo 3 - DDA TIC Proteinas \\
\hline 492 & Grafo 3 - DDA TIC Proteinas \\
\hline 4881 & Grafo 3 - DDA TIC Proteinas \\
\hline 802 & Grafo 3 - DDA TIC Proteinas \\
\hline 251 & Grafo 3 - DDA TIC Proteinas \\
\hline 2134 & Grafo 3 - DDA TIC Proteinas \\
\hline
\end{tabular}


Tabela A.25 - Estimativas das componentes de variância entre os experimentos avaliados

\begin{tabular}{lrrrr}
\hline Experimento & $u_{1}$ & $u_{2}$ & $u_{3}$ & Erro \\
\hline SRM & 0,0894 & 0,0615 & 0,0009 & 0,4125 \\
DDA noNorm Peptídeos & 0,0366 & 0,0333 & 0,0440 & 0,7177 \\
DDA noNorm Proteínas & 0,0430 & 0,0391 & 0,0440 & 0,7916 \\
DDA TIC Peptídeos & 0,0285 & 0,0261 & 0,0092 & 0,7177 \\
DDA TIC Proteínas & 0,0440 & 0,0223 & 0,0003 & 0,7924 \\
\hline
\end{tabular}

